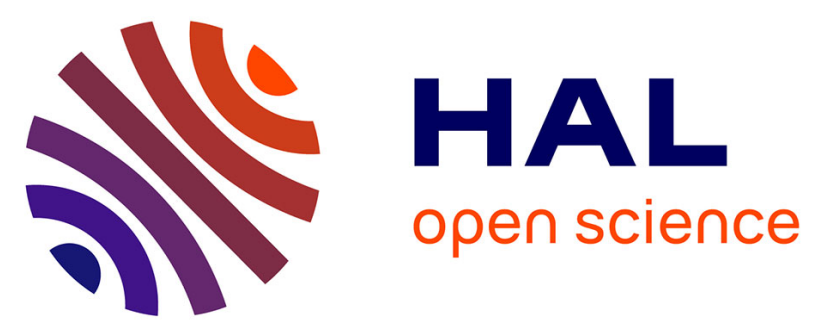

\title{
Les Bagnoles à l'Isle-sur-la-Sorgue (Vaucluse) : aspects nouveaux dans le Néolithique moyen du midi de la France
}

\author{
Jean-Philippe Sargiano, Samuel van Willigen, André d'Anna, Stéphane \\ Renault, Katja Hunger, Marie Woerle-Soares, Robert Gaday
}

\section{To cite this version:}

Jean-Philippe Sargiano, Samuel van Willigen, André d'Anna, Stéphane Renault, Katja Hunger, et al.. Les Bagnoles à l'Isle-sur-la-Sorgue (Vaucluse) : aspects nouveaux dans le Néolithique moyen du midi de la France. Gallia Préhistoire - Préhistoire de la France dans son contexte européen, 2010, 52, pp.193-239. 10.3406/galip.2010.2473 . halshs-00585222

\section{HAL Id: halshs-00585222 \\ https://shs.hal.science/halshs-00585222}

Submitted on 2 Jan 2020

HAL is a multi-disciplinary open access archive for the deposit and dissemination of scientific research documents, whether they are published or not. The documents may come from teaching and research institutions in France or abroad, or from public or private research centers.
L'archive ouverte pluridisciplinaire HAL, est destinée au dépôt et à la diffusion de documents scientifiques de niveau recherche, publiés ou non, émanant des établissements d'enseignement et de recherche français ou étrangers, des laboratoires publics ou privés.

\section{(ㅇ)(1) $\$$}

Distributed under a Creative Commons Attribution - NonCommercial - NoDerivatives 44.0 


\title{
LES BAGNOLES
}

\section{À L'ISLE-SUR-LA-SORGUE (VAUCLUSE)}

\section{Aspects nouveaux dans le Néolithique moyen du midi de la France}

\author{
Jean-Philippe SARgiano ${ }^{1}$, Samuel VAn Willigen ${ }^{2}$, André D’Anna ${ }^{3}$, \\ Stéphane RENAUlT ${ }^{3}$, Katja HUNGER ${ }^{4}$, Marie WOERLE-SOARES ${ }^{4}$ et Robert GADAY ${ }^{5}$
}

\begin{abstract}
Mots-clés. Chasséen, céramique, industrie lithique.
Résumé. La réalisation d'un diagnostic archéologique au lieu-dit les Bagnoles (commune de l'Isle-sur-la-Sorgue, Vaucluse) durant l'été 2006 a conduit à la découverte d'un ensemble de structures en creux attribuables au Néolithique moyen. Bien que le site ne soit connu que de manière très partielle, il peut apparaître d'ores et déjà comme important. En effet, plusieurs de ces ensembles clos relèvent non seulement d'un aspect du Chasséen jusqu'à présent mal identifié dans le midi de la France, mais ils ont également livré des formes et des décors céramiques inédits dans le Néolithique moyen régional.
\end{abstract}

Key-words. Chassean, pottery, lithic industry.

Abstract. The realization of a diagnostic archaeology operation in the hamlet of Bagnoles (commune of Isle-sur-Sorgue, Vaucluse) during the summer of 2006 led to the discovery of a group of hollow structures attributable to the Middle Neolithic. Though this site is known only very partially, it already appears to be significant. Indeed, several of these closed groups of structures reveal not only an aspect of the Chassean poorly identified until now in the Midi region of France, but have also yielded pottery forms and decorations unknown in the regional Middle Neolithic.

Translation: Magen O'FARRELL

Découvert en 2006 à l'occasion d'un diagnostic, le site des Bagnoles a livré un mobilier comparable à celui mis au jour ces dernières années sur une série de sites du midi de la France et généralement attribué à un aspect considéré comme ancien du Chasséen.
En dépit de recherches très partielles, conséquence des conditions de fouilles, l'importance de cette découverte nous a conduits à choisir de mettre rapidement les données relatives au mobilier archéologique à la disposition de la communauté scientifique. Il s'agit donc d'une présentation

1. Inrap Méditerranée, 24 avenue de la Grande-Bégude, F-13770 Venelles et Lampea, UMR 6636, Université de Provence. Courriel: jean-philippe. sargiano@inrap.fr

2. Lampea, UMR 6636, Université de Provence, Maison méditerranéenne des sciences de l'homme, 5 rue du Château-de-l'Horloge, BP 647, F-13094 Aix-en-Provence Cedex 2 et Musée national suisse, Zurich. Courriel: samuel.vanwilligen@snm.admin.ch

3. Lampea, UMR 6636, Université de Provence, Maison méditerranéenne des sciences de l'homme, 5 rue du Château-de-l'Horloge, BP 647, F-13094 Aix-en-Provence Cedex 2. Courriels: danna@mmsh.univ-aix.fr; renault@mmsh.univ-aix.fr

4. Musée national suisse, Centre des collections, Lindenmoosstrasse 1, CH-8910 Affoltern am Albis. Courriels: katja.hunger@snm.admin.ch; marie.woerle@snm.admin.ch

5. Inrap Méditerranée, 24 avenue de la Grande-Bégude, F-13770 Venelles. Courriel: robert.gaday@inrap.fr 
préliminaire qui n'aborde que certains des principaux aspects du site.

Cependant, au-delà de la présentation des assemblages, la nature du mobilier recueilli dans certaines structures nous conduit à nous interroger sur son insertion chronoculturelle au sein du Néolithique moyen du midi de la France. Les comparaisons possibles sont rapidement énumérées; elles débouchent sur des questions relatives à la signification que les néolithiciens donnent au terme de «Chasséen » ainsi que sur le problème de sa définition en tant que culture archéologique.

Il n'est pas question ici de proposer une modification complète de la structure du Néolithique moyen méridional. Néanmoins, vingt ans après les derniers consensus (Boutié, 1988; Beeching et al., 1991), celle-ci nécessite à notre sens une certaine révision. Actuellement, le nombre croissant d'ensembles clos (remplissages de fosses et sépultures), véritables «instantanés» de la culture matérielle, et la multiplication des datations radiocarbone devraient permettre de préciser la classification du Néolithique moyen méditerranéen.

\section{LE SITE, LES STRUCTURES ET LE MOBILIER}

\section{LE SITE}

Le projet de création d'une plateforme logistique pour le transport routier dans la plaine de la Sorgue a occasionné en juin et juillet 2006 la réalisation par l'Inrap d'un diagnostic archéologique sous la direction de Robert Gaday ${ }^{6}$. L'opération a comporté 52 sondages en tranchées de $2 \mathrm{~m}$ de large (Gaday, Sargiano, 2006). La surface explorée représente $9284 \mathrm{~m}^{2}$ sur un espace à diagnostiquer de $210000 \mathrm{~m}^{2}$; aucun site archéologique n'était initialement mentionné sur les parcelles concernées et leurs abords. Le projet d'aménagement ayant été abandonné, le diagnostic n'a été suivi d'aucune fouille de sauvetage.

Le site est implanté à 2,5 km au sud-ouest de l'Isle-sur-laSorgue et à environ 20 km à l'est-sud-est d'Avignon (fig. 1). Il est localisé sur les alluvions fluviatiles, probablement würmiennes, de l'ancien cours de la Durance, mêlées à celles du Calavon et de la Sorgue, qui constituent ici les riches terres agricoles de la plaine du Comtat, à l'extrémité occidentale

6. Réalisée du 30 mai au 25 juillet 2006 par cinq archéologues de terrain, l'opération a été financée par la Communauté de communes du pays des Sorgues et des monts de Vaucluse à la suite d'une autosaisine. des plateaux de Vaucluse et du Grand Luberon. Ces terrains sont relativement plats en situation basse entre $55 \mathrm{~m}$ et $60 \mathrm{~m}$ d'altitude NGF. Ils sont traversés selon un axe globalement nord-ouest-sud-est par une «roubine ${ }^{7}$ ». Elle est liée à un ensemble de petits cours d'eau (le Petit Mourgon, le Grand Mourgon) appartenant au système hydrologique des Sorgues qui traversent des terrains anciennement mal drainés, ce dont témoigne clairement le toponyme «les Bagnoles » qui désigne un lieu humide. Cette roubine divise l'espace en deux secteurs principaux, l'un au nord de la ferme des Bagnoles, au nord-est et à l'est de l'emprise évaluée et l'autre au sud-ouest. Le premier de ces deux secteurs sera dénommé nord-est dans les descriptions ci-dessous et le second sud-ouest.

Sur tout l'espace sondé, la séquence sédimentaire est de même nature, mais les couches présentent des variations latérales en termes de présence/absence et d'épaisseur: elles sont plus dilatées vers le nord-est. La séquence est constituée de quatre ensembles lithologiques avec de bas en haut:

- une argile limoneuse jaune-gris et plus rarement verdâtre dans laquelle des lentilles de sables ou de graviers indiquent la présence de paléochenaux; cet ensemble constitue le substratum du site;

- un limon sableux jaune et gris, localement faiblement argileux;

- un limon argileux beige induré à nodules de calcaire et à taches orange ou blanches ;

- un limon brun montrant des cailloux et des gravillons épars, dont la partie supérieure constitue les terres labourées.

Dans l'ensemble des sondages, cent deux structures en creux et anomalies ont été repérées. Toutes n'ont pas été fouillées. Leur répartition n'est pas uniforme et on peut reconnaître trois zones de concentration, auxquelles il convient d'ajouter quelques structures isolées. Cette organisation reste probablement largement tributaire de la nature de l'intervention.

Seules les structures ayant fourni du mobilier significatif et diagnostique sont décrites dans cette note (fig. 2). La première partie livre les données brutes et vise à identifier et présenter les ensembles clos. Une présentation, objet par objet et non globale, s'avère en effet nécessaire afin de fonder des comparaisons fiables. Cette description est suivie d'une discussion relative à l'attribution culturelle

7. De l'occitan robina: canal de drainage ou de dérivation. 


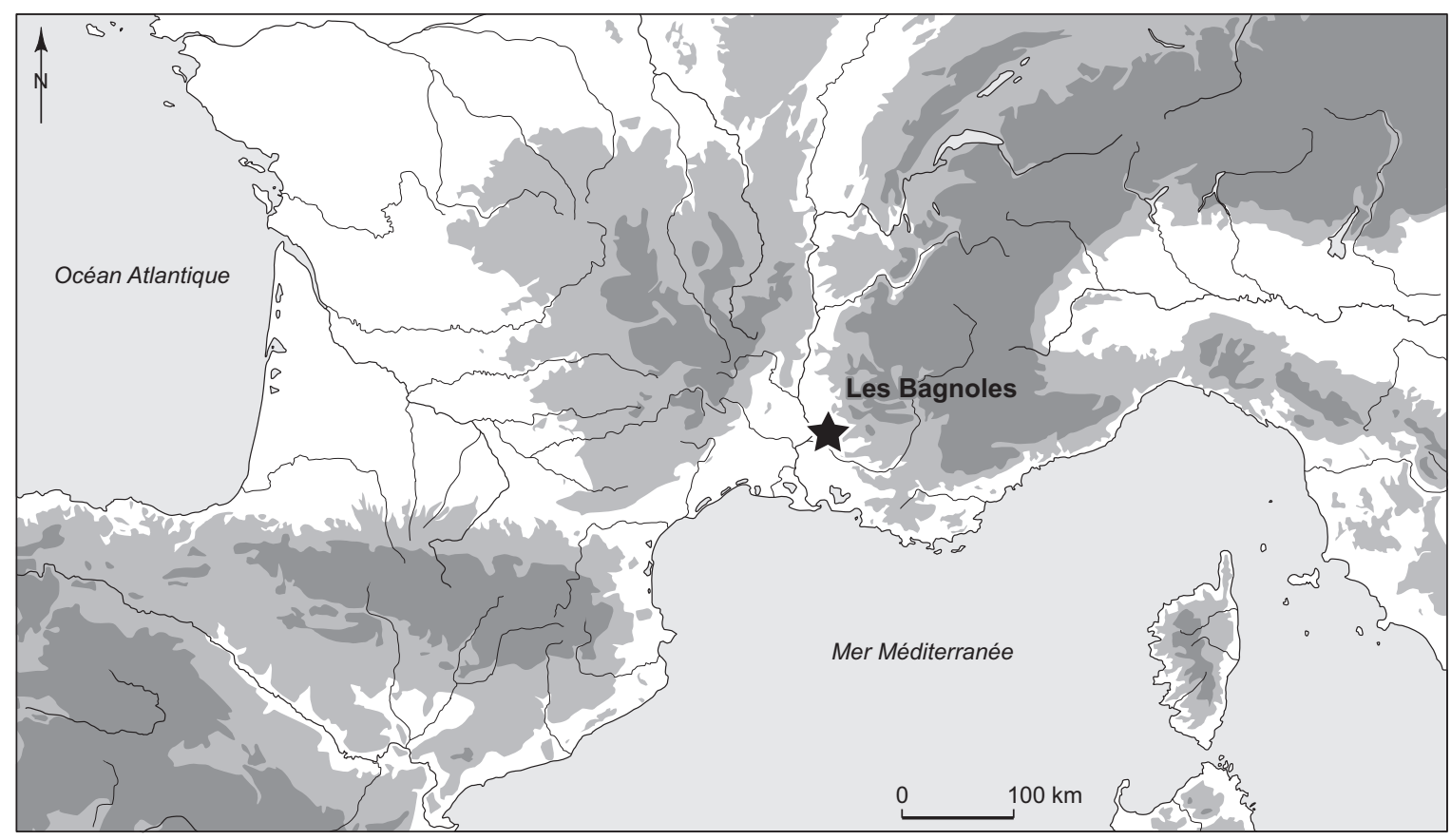

Fig. 1 - Localisation du site des Bagnoles à l'Isle-sur-la-Sorgue (Vaucluse) (DAO: S. Van Willigen, Lampea).

des ensembles ainsi définis et aux questions soulevées par certains d'entre eux.

\section{LES STRUGTURES ET LEUR MOBILIER}

Dans l'état actuel des connaissances, douze structures ou unités stratigraphiques ont livré du mobilier néolithique (tabl. I). En l'absence d'échantillons appropriés (charbons de bois ou ossements non brûlés) dans la majorité des structures fouillées, il n'a pas été possible de réaliser des datations radiométriques. Seules des comparaisons avec d'autres ensembles clos bien datés permettent de discuter la chronologie des structures et de poser les bases de la discussion. D’une manière générale, l'industrie lithique du site est réalisée sur du silex bédoulien dont la provenance précise reste incertaine: monts de Vaucluse, région de Châteauneuf-du-Pape ou encore région du mont Ventoux. Dans les descriptions, nous ne préciserons que l'origine probable des autres matières premières.

\section{STRUCTURE SI 01}

Située au nord-est de l'emprise, la structure SI 01 est isolée dans la tranchée de sondage TR 05 (fig. 2). Elle s'ouvre à $1,30 \mathrm{~m}$ sous le sol actuel. Le diamètre est de 1,28 m à l'ouver-
Tabl. I - Liste des structures des Bagnoles attribuables au Néolithique moyen (Ø: aucun élément; ?: mobilier atypique) (DAO: S. Van Willigen, Lampea).

\begin{tabular}{|c|c|c|}
\hline $\mathbf{N}^{\circ}$ structure & Lithique & Céramique \\
\hline SI 01 & $?$ & Post-Chasséen \\
\hline FS 13 & $?$ & Chasséen \\
\hline FO 14 & Néolithique moyen & $\varnothing$ \\
\hline FS 22 & Néolithique moyen (?) & Chasséen (?) \\
\hline US 31 & Néolithique moyen & $\varnothing$ \\
\hline US 42 & $?$ & Néolithique moyen (?) \\
\hline FS 47 & $?$ & Chasséen \\
\hline FS 48 & $\varnothing$ & Chasséen \\
\hline US 62 & Néolithique moyen (?) & Post-Chasséen (?) \\
\hline US 67 & Néolithique moyen & $\varnothing$ \\
\hline US 70 & $?$ & Post-Chasséen (?) \\
\hline FS 75 & Néolithique moyen (?) & Chasséen \\
\hline
\end{tabular}

ture, de $0,70 \mathrm{~m}$ à $0,50 \mathrm{~m}$ de profondeur et de $1,30 \mathrm{~m}$ à $0,75 \mathrm{~m}$ de profondeur. La profondeur totale est de 1,02 m. Le profil global est piriforme, creusé dans les limons sableux jaunes; le fond et les parois sont irréguliers.

Le remplissage comporte trois ensembles: à la base un limon argileux brun-gris à taches rousses contenant des pierres et du mobilier archéologique, dans la partie médiane un limon argileux jaune et gris comprenant des pierres, 


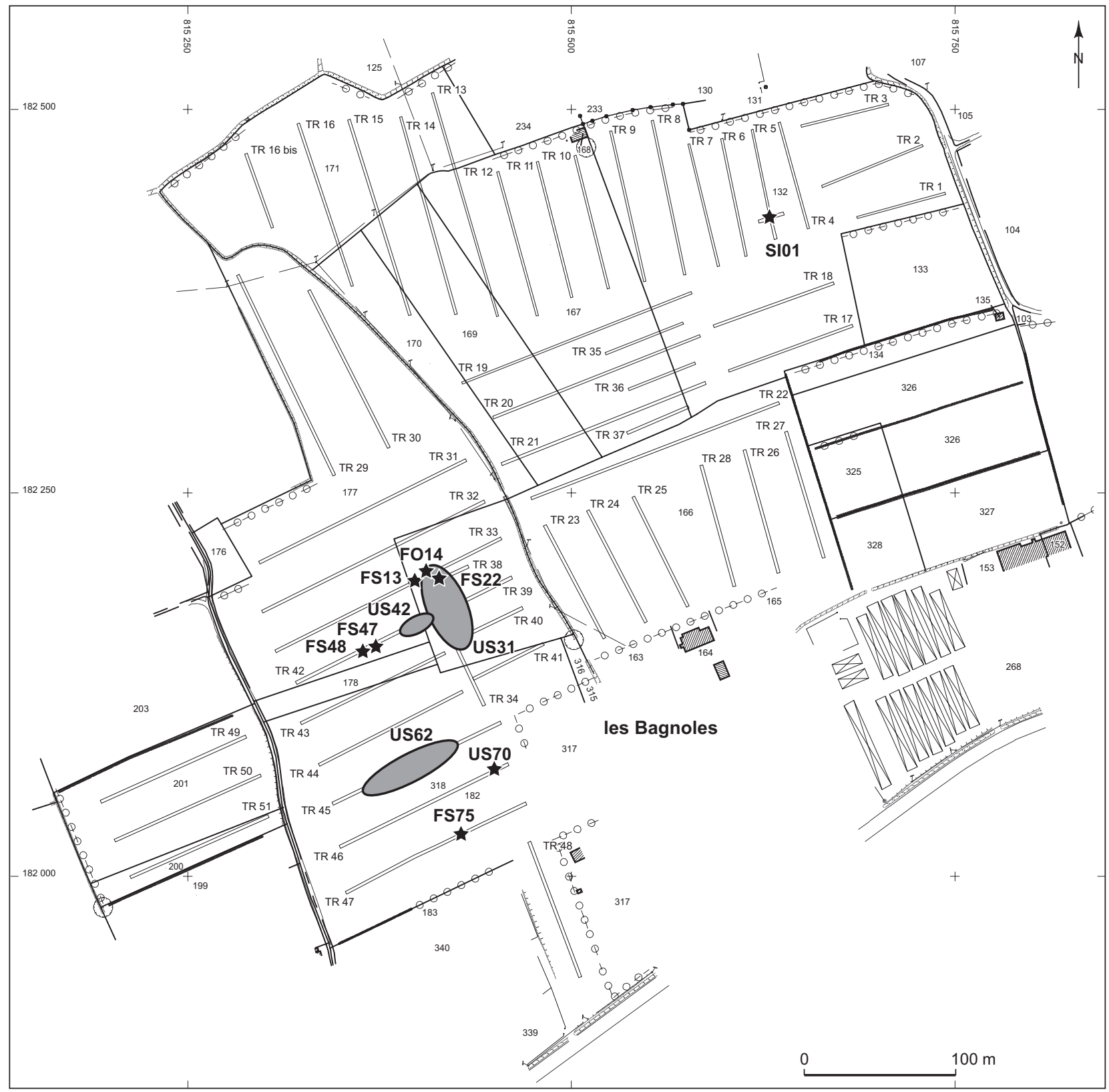

Fig. 2 - Plan général avec la localisation des structures ayant livré du mobilier néolithique moyen (SI: silo; FS: fosse; FO: fossé; US: unité stratigraphique) (DAO: R. Gaday, Inrap).

dans la partie supérieure un limon très argileux gris verdâtre, incluant de la céramique et des coquilles d'escargots.

Cette structure a livré dix-huit fragments de céramique (dont cinq jointifs) appartenant à au moins onze vases différents dont deux formes reconstituables: une jatte carénée à col concave vertical (fig. $3, n^{\circ} 2$ ) et une jatte à carène vraisemblablement basse et col concave vertical et partie inférieure concave (fig. $3, \mathrm{n}^{\circ} 1$ ).

L'industrie lithique est peu abondante. Elle se résume à deux objets: un petit galet qui a pu être utilisé comme abraseur (?) et un fragment mésial de lame en silex brun clair qui provient de la partie inférieure du remplissage; la 

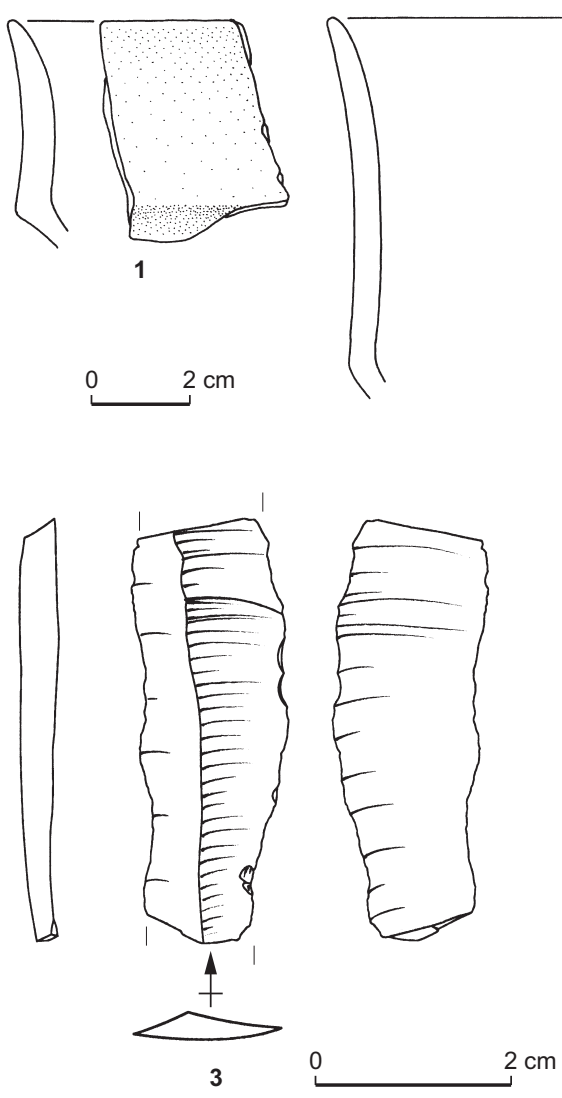

section est triangulaire, les nervures et les bords sont irréguliers, la face inférieure porte des ondulations marquées (fig. 3, no 3).

\section{STRUCTURE FS 13}

La fosse FS 13 est localisée dans la partie centrale du secteur sud-ouest. Elle apparaît à $0,65 \mathrm{~m}$ sous le sol actuel. De forme rectangulaire, elle a une orientation nord-sud qui se poursuit sous la berme de la tranchée de sondage. La longueur reconnue est de 0,82 $\mathrm{m}$ et la largeur de 0,35 m. Creusée dans l'argile limoneuse, elle a un comblement constitué de limon argileux brun comprenant des pierres et des graviers.

Les quarante-neuf fragments de céramique (dont neuf jointifs) correspondent à un minimum de quatorze vases. Les neuf tessons diagnostiques appartiennent à six individus différents: un vase de $16 \mathrm{~cm}$ de diamètre, à lèvre amincie, profil légèrement galbé, inflexion basse et col subvertical (fig. 4, $\mathrm{n}^{\mathrm{O}} 1$ ) avec un trou de réparation situé à environ 1,5 cm du bord; un fragment de bord pourrait correspondre à un autre vase de forme et de dimension comparables (fig. 4, $\mathrm{n}^{\circ} 2$ ); deux fragments de bord appartiennent à des vases de dimension moyenne et de forme indéterminée (fig. 4, nos 3-4); quatre tessons à dégraissant grossier constitué de grains de calcite permettent de reconstituer partiellement une petite coupe en calotte à bord arrondi et épaississement interne (fig. 4, $\mathrm{n}^{\circ} 5$ ); une poignée large et courbe (fig. 4, $\mathrm{n}^{\circ} 6$ ).

L'industrie lithique comprend dix-sept objets principalement en silex taillé. Une lame de pierre polie subtriangulaire en roche verte (probablement une éclogite) cassée et réaffûtée (longueur: $5,9 \mathrm{~cm}$, largeur: $1 \mathrm{~cm}$ à $3,2 \mathrm{~cm}$, épaisseur: $0,8 \mathrm{~cm}$ à $2 \mathrm{~cm}$ ) est un des rares objets de ce type trouvé sur le site (fig. 4, $\mathrm{n}^{\mathrm{o}} 7$ ). L'industrie taillée en silex comporte les éléments suivants: un fragment de nucléus qui porte des retouches semi-abruptes; six éclats et éclats laminaires (fig. 4, no 8), ainsi que deux débris; une tablette à retouches inverses et opposées, plates, courtes à longues, proximales et distales (pièce esquillée ?) (fig. 4, $\mathrm{n}^{\circ} 9$ ) ; un grattoir sur éclat épais à retouche semi-abrupte et abrupte denticulée longue (fig. 5, $\mathrm{n}^{\mathrm{o}} 1$ ) ; un grattoir sur éclat laminaire épais à talon 

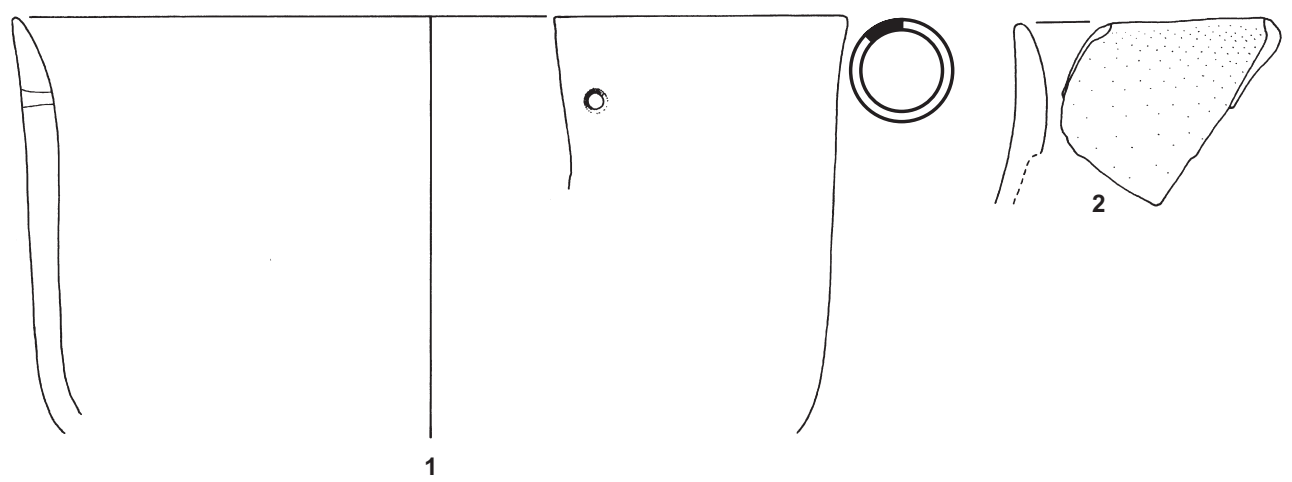

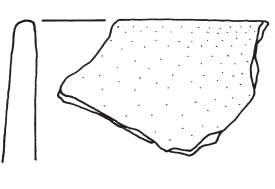

3
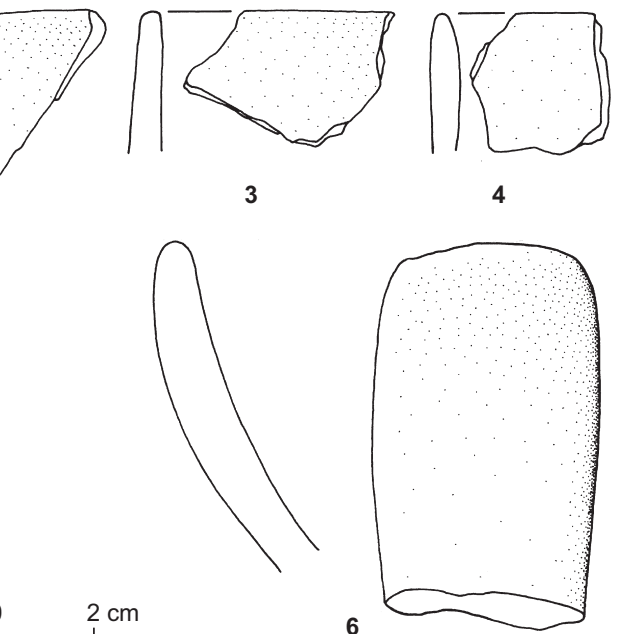

$2 \mathrm{~cm}$
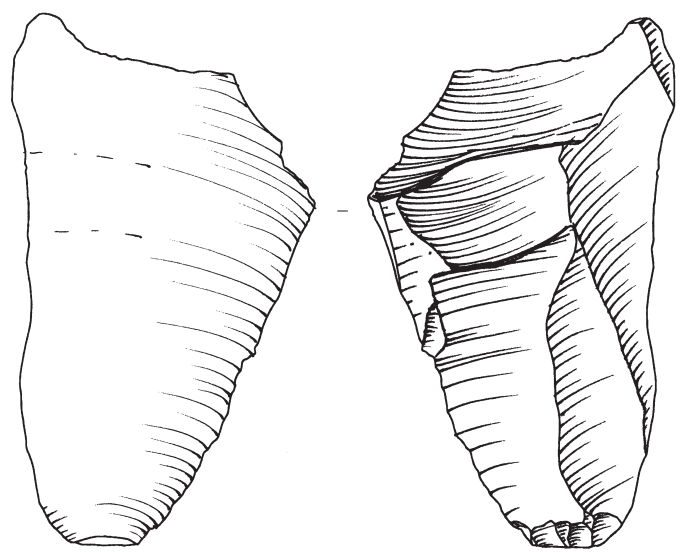

8

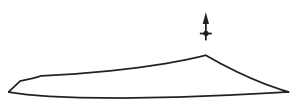

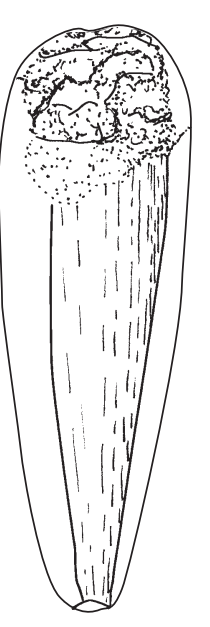
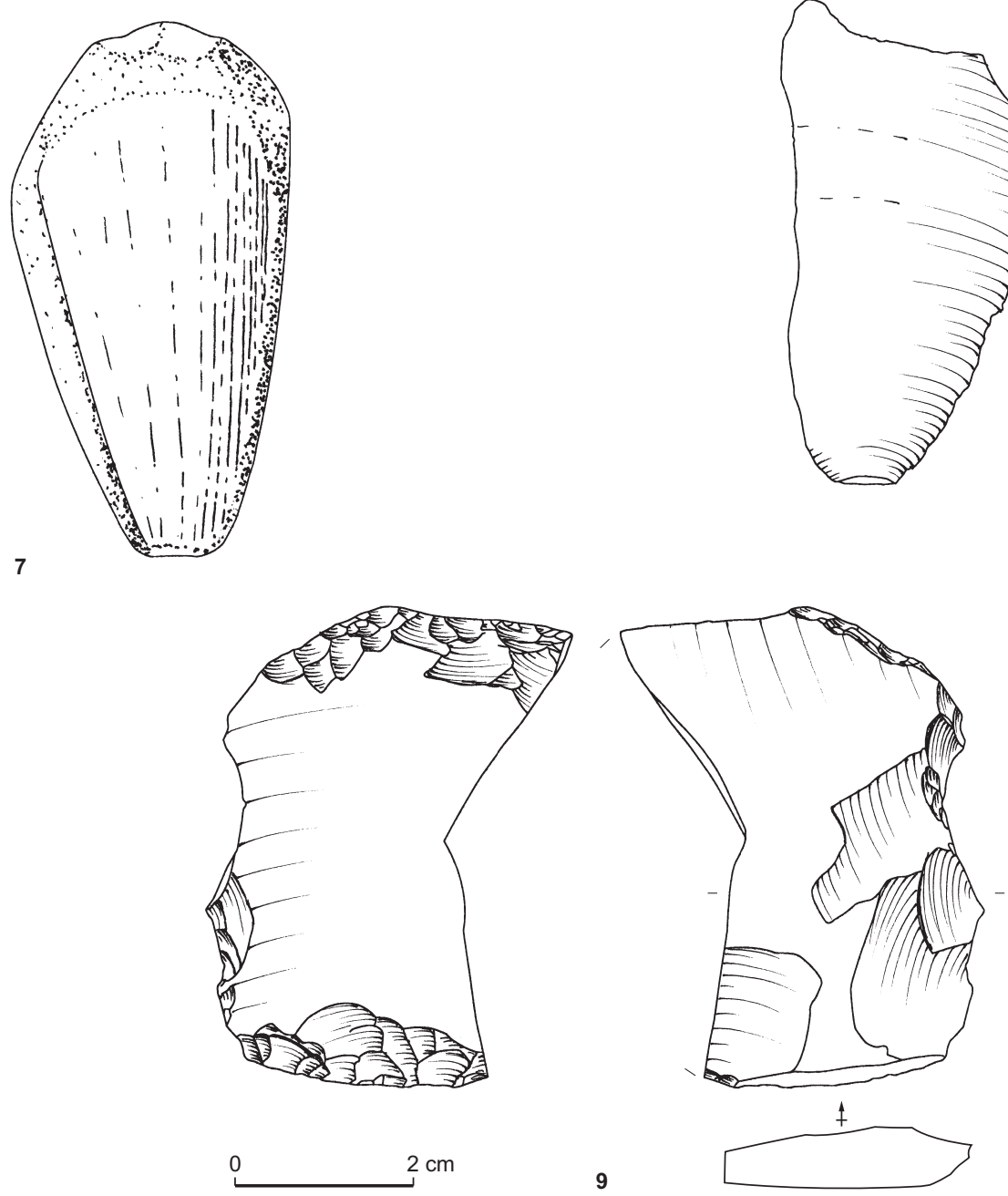

Fig. 4 - Mobilier céramique et industrie lithique de la structure FS 13 (dessin: S. Renault, CNRS et S. Van Willigen, Lampea). 

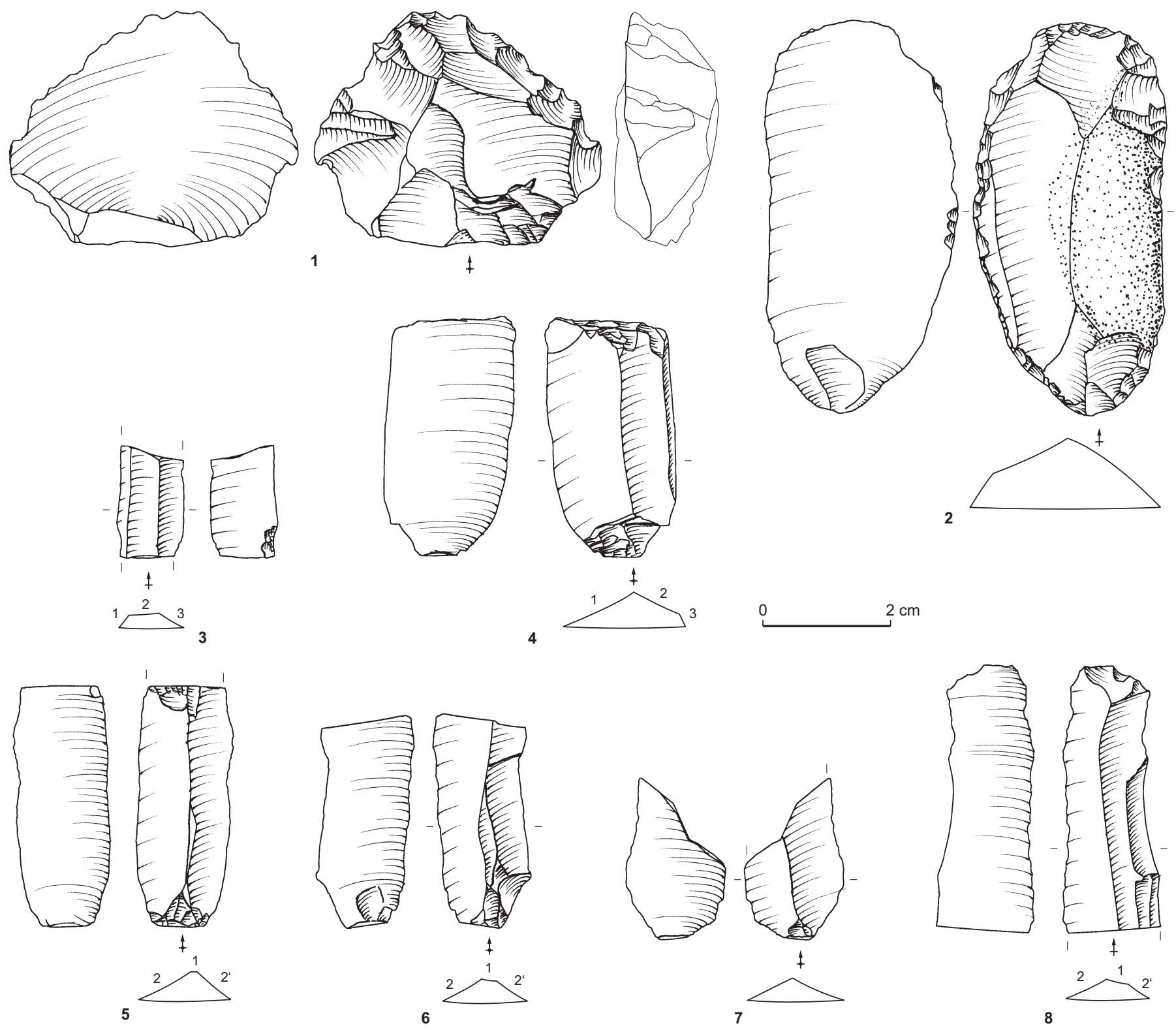

Fig. 5 - Industrie lithique de la structure FS 13 (dessin: S. Renault, CNRS).

écrasé, bulbe esquillé, à bord droit partiellement cortical et dont la partie proximale porte des retouches courtes à longues, semi-abruptes à obliques: le bord gauche présente des retouches courtes, le front du grattoir porte deux séries d'enlèvements, longs et semi-abrupts et une série d'enlèvements subparallèles courts (fig. 5, no 2); un fragment mésial de lamelle à section trapézoïdale asymétrique, nervures et bords rectilignes et parallèles (fig. 5, no 3); une courte lame large rebroussée dont le plan de frappe a été préparé par la réduction de la corniche, la section est trapézoïdale asymétrique, les nervures et bords relativement rectilignes et parallèles (un bord flottant) : le bulbe montre un cône et une ridule et la face inférieure une ondulation, l'extrémité distale porte des retouches directes, irrégulières et obliques (fig. 5, no 4); un fragment proximal de lame dont le plan de frappe a été préparé par doucissage, la face inférieure porte une lèvre, la largeur maximale est rapidement atteinte, la section est triangulaire, les nervures et les bords ne sont pas parfaitement rectilignes (fig. 5, $\mathrm{n}^{\circ} 5$ ); une lame torse, à talon relativement gros, la corniche importante n'a subi 

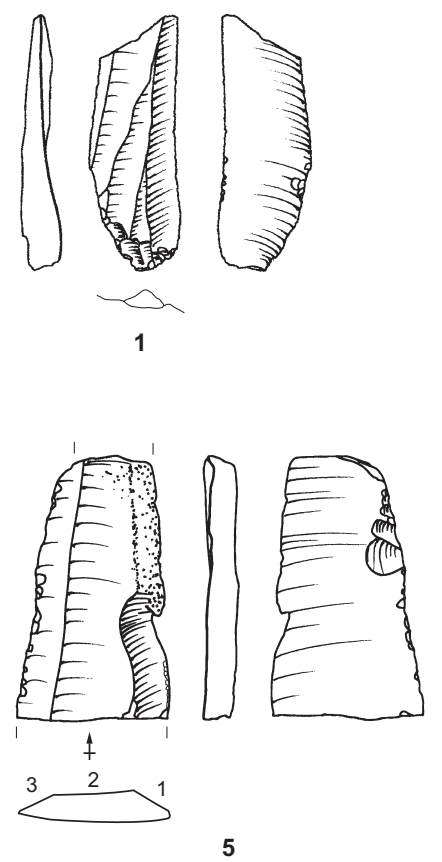
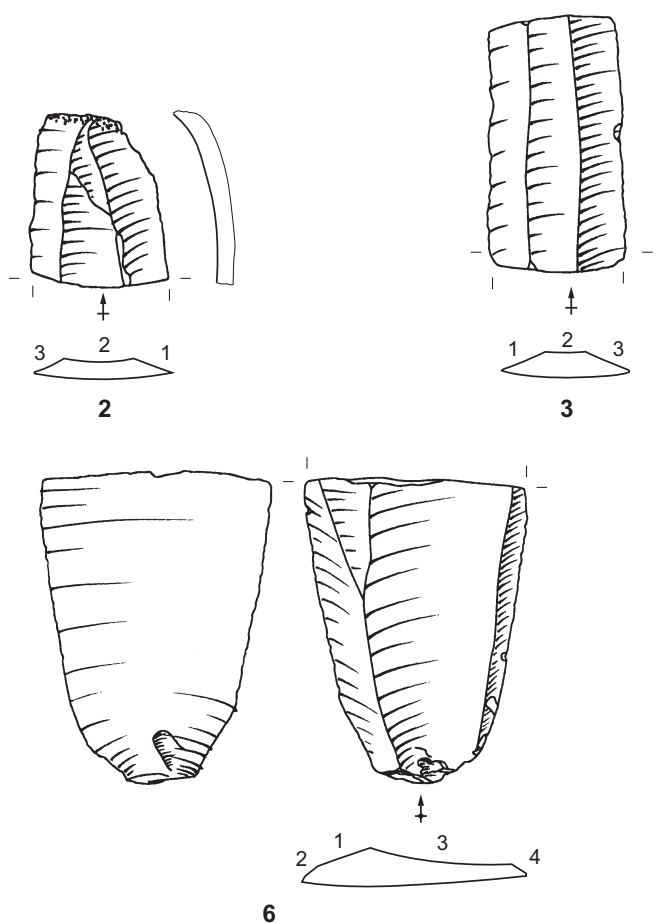
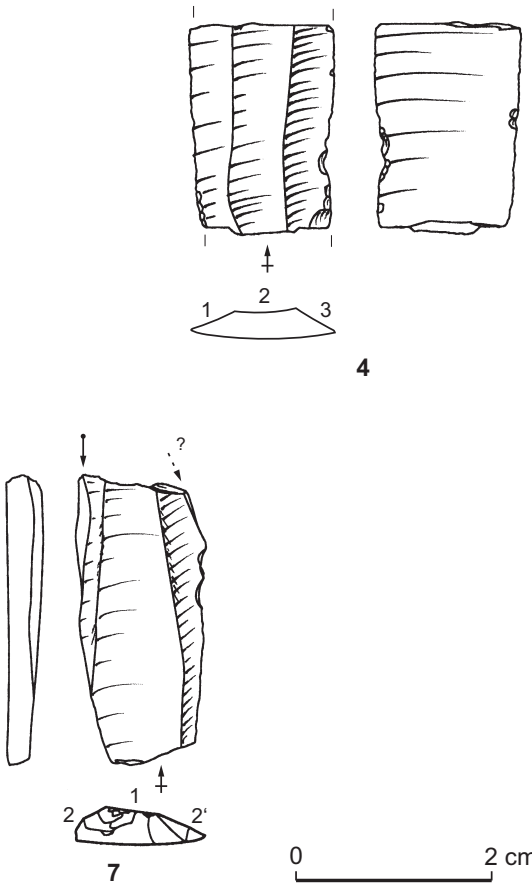

7 $2 \mathrm{~cm}$

Fig. 6 - Industrie lithique de la structure FO 14 (dessin: S. Renault, CNRS).

qu'un doucissage, la section est trapézoïdale et triangulaire, les nervures et les bords sont irréguliers (fig. 5, $\mathrm{n}^{\mathrm{o}} 6$ ); un fragment proximal de lamelle (fig. $5, \mathrm{n}^{\circ} 7$ ) ainsi qu'un fragment distal de lamelle de section trapézoïdale (fig. 5, no 8 ).

\section{STRUCTURE FO 14}

Localisée dans la partie centrale du secteur sud-ouest, ce tronçon de fossé, large de 1,80 m et orienté nord-est-sudouest a été reconnu à $0,60 \mathrm{~m}$ de profondeur. Il est creusé dans l'argile limoneuse et son comblement est constitué d'un limon brun contenant des graviers, des cailloux, des nodules calcaires et du mobilier.

Cette structure n'a livré aucun tesson diagnostique ${ }^{8}$. L'industrie lithique comporte douze objets patinés (fig. 6) : un nucléus cortical et brûlé; trois éclats et trois débris; un fragment proximal de lamelle torse (fig. $6, \mathrm{n}^{\circ} 1$ ); un fragment distal de lame outrepassée à section trapézoïdale (fig. 6, no 2); deux fragments mésiaux de lames à section

8. Tout fragment de bord, de fond, de préhension et/ou de décor. Nous préférons l'expression «tesson diagnostique» aux termes «typique», «caractéristique» ou encore «forme», certes plus usités mais qui peuvent porter à confusion. En effet, un fragment de bord ou de préhension n'est pas nécessairement caractéristique et ne permet pas toujours de reconstituer une forme. trapézoïdale (fig. $6, \mathrm{n}^{\text {os }} 3$ et 4 ), à bords et nervures rectilignes relativement parallèles (code de débitage 1-2-3); un fragment mésial de lame à bord droit cortical et à section en partie trapézoïdale (fig. $6, \mathrm{n}^{\circ} 5$ ); un fragment proximal de lame (fig. $6, \mathrm{n}^{\circ} 6$ ), le plan de frappe a été préparé par doucissage, le bulbe peu prononcé comporte une lèvre et un esquillement, la section est trapézoïdale, la largeur a été mal contrôlée (fine en partie proximale, elle s'évase en partie mésiale) (code de débitage 1-2-3-4); un burin sur troncature par retouche abrupte inverse, sur le bord droit d'une lame à section trapézoïdale et nervures rectilignes convergentes (fig. 6, no 7; code de débitage 2-1-2').

\section{STRUCTURE FS 22}

La fosse FS 22, toujours dans le même secteur (fig. 2), a été reconnue à $0,65 \mathrm{~m}$ sous le sol actuel. De forme allongée, orientée nord-sud, elle a une longueur de 1,20 m et une largeur de 0,50 m. Elle est creusée dans l'argile limoneuse, son comblement est constitué d'un limon argileux comprenant des pierres, des graviers et du mobilier archéologique.

Les vingt-huit fragments de céramique (dont quatorze jointifs) appartiennent à un minimum de cinq vases. Dixneuf tessons permettent de reconstituer partiellement un vase à profil légèrement galbé, lèvre amincie et inflexion 

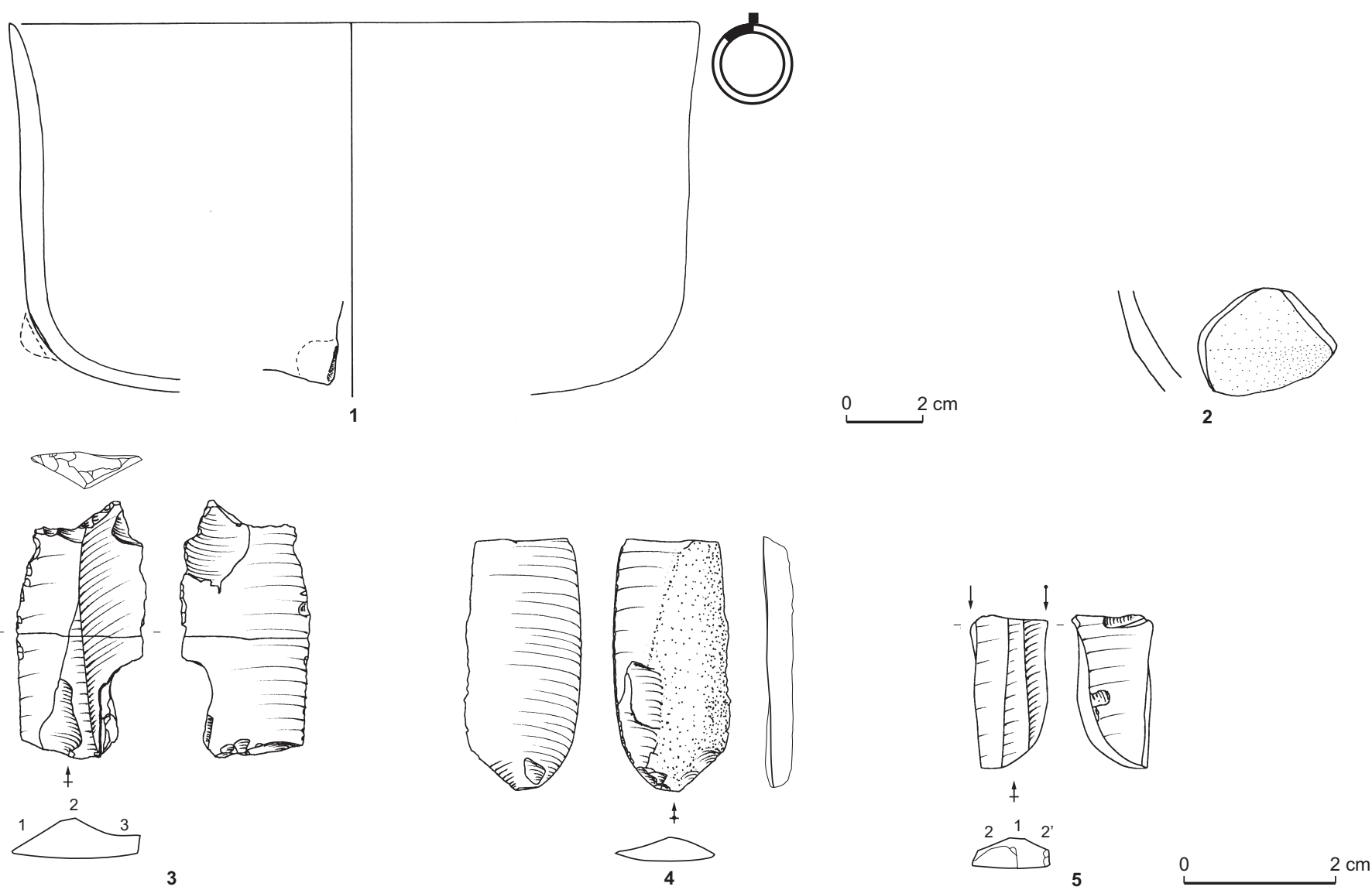

Fig. 7 - Mobilier céramique et industrie lithique de la structure FS 22 (dessin: S. Renault, CNRS et S. Van Willigen, Lampea).

basse, d'un diamètre de $17 \mathrm{~cm}$ pour une hauteur approximative de $10 \mathrm{~cm}$ (fig. $7, \mathrm{n}^{\mathrm{O}} 1$ ). La trace d'un bouton à perforation verticale décollé est visible à hauteur de l'inflexion de la paroi. Ce vase est en tout point comparable aux vases à profil galbé et inflexion basse des structures 13 et 75. Un fragment de panse correspond à un autre vase qui présentait une légère carène (fig. 7, no 2).

L'industrie lithique comporte sept objets en silex: un éclat cortical brûlé et un débris; un éclat ayant emporté une partie de la surface de débitage avec une retouche alterne sur une extrémité; un fragment distal cortical et brûlé d'éclat ou de lame; un fragment mésial de lame patinée en silex zoné tertiaire à section triangulaire avec un burin rebroussé sur troncature sur le bord droit (fig. 7, no 3); un fragment proximal de lame corticale de section triangulaire dont le plan de frappe a été préparé par doucissage (fig. 7, $\mathrm{n}^{\mathrm{o}} 4$ ), la face inférieure porte une lèvre, un esquillement sur le bulbe et de très légères ondulations, le bord gauche porte des retouches grignotées; un burin double distal, outrepassé sur le bord droit, sur fragment mésial de lame à section trapézoïdale et nervures régulières et légèrement convergentes: l'objet est entièrement patiné (fig. $7, \mathrm{n}^{\circ} 5$ ).

\section{UNITÉ STRATIGRAPHIQUE US 31}

L'unité stratigraphique US 31 a été reconnue à $0,55 \mathrm{~m}$ sous le sol actuel. Elle est constituée par un niveau de limon argileux brun foncé d'environ $0,10 \mathrm{~m}$ à $0,15 \mathrm{~m}$ d'épaisseur, contenant des pierres, des graviers localement concentrés en «nappes» et du mobilier. Cette couche archéologique semble continue sur une surface relativement étendue puisqu'elle a été reconnue dans plusieurs tranchées de sondage relativement proches de la limite est de ce secteur (TR 33, TR 34, TR 38, TR 39 et TR 40).

Cette structure n'a livré aucun fragment céramique diagnostique. L'industrie lithique comporte dix-neuf objets: six éclats (dont deux corticaux) et un débris; un nucléus à éclats (fig. 8, $\mathrm{n}^{\mathrm{o}} 1$ ); un fragment mésial de lamelle en 

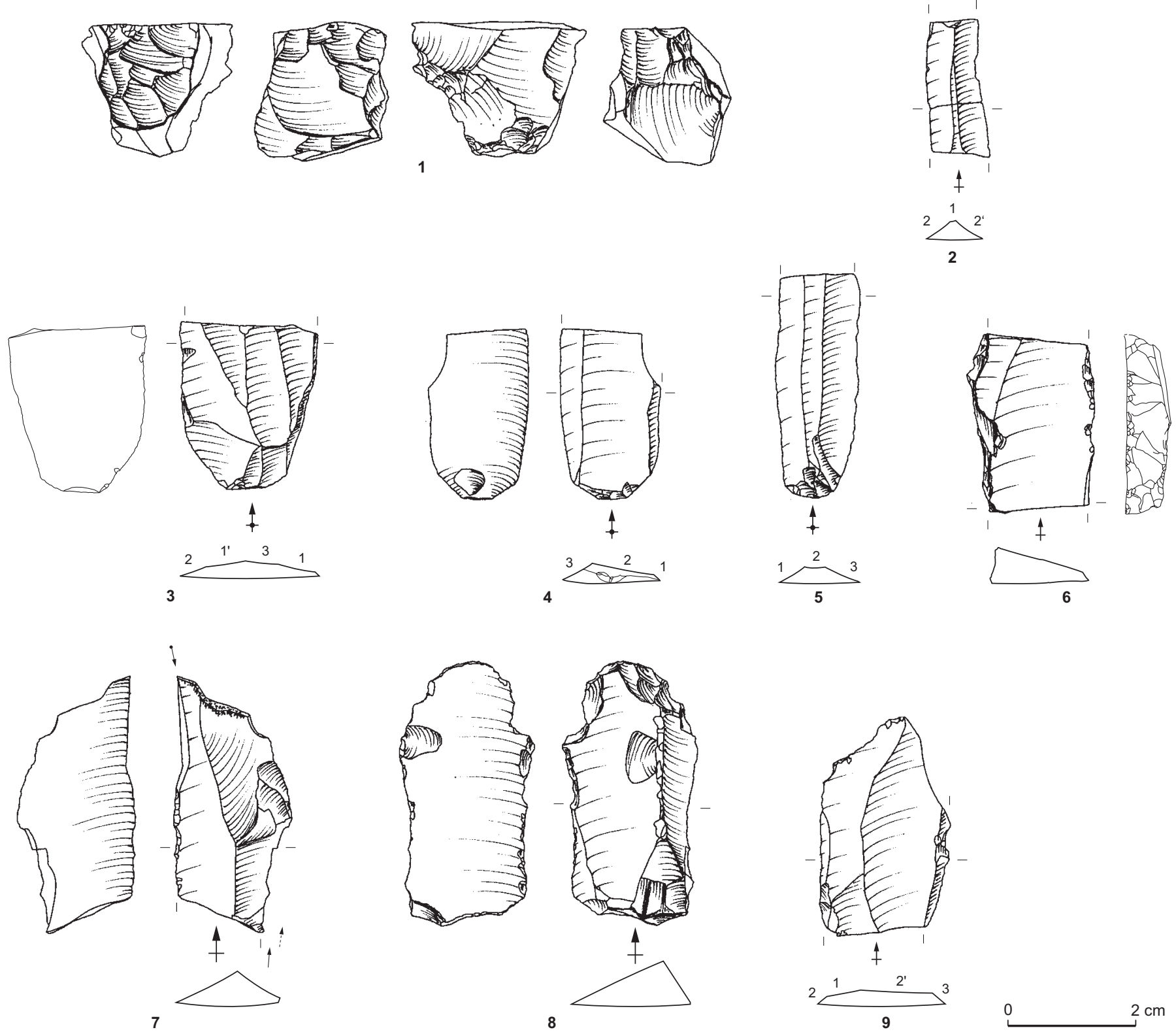

$2 \mathrm{~cm}$

Fig. 8 - Industrie lithique de l'unité stratigraphique US 31 (dessin: S. Renault, CNRS).

silex tertiaire à section trapézoïdale puis triangulaire, à bords et nervures relativement rectilignes et parallèles (fig. 8, $\mathrm{n}^{\mathrm{o}}$ 2); un fragment de lame brûlée; un fragment proximal de lame torse à cinq versants, le plan de frappe a été préparé par abrasion et doucissage, l'angle de chasse est aigu, le bulbe comporte une petite lèvre (fig. $8, \mathrm{n}^{\mathrm{o}} 3$ ) ; deux fragments proximaux patinés de lames de section trapézoïdale (une asymétrique, l'autre symétrique), le plan de frappe a été préparé par doucissage pour l'un (fig. 8, no 4 ) et par abrasion et doucissage pour l'autre (fig. 8, $\mathrm{n}^{\circ} 5$ ), le bulbe est haut et court et porte une lèvre, les bords et les nervures sont rectilignes et parallèles (code de débitage 1-2-3) ; un fragment mésial de lame épaisse à bord abattu par retouche croisée sur le bord droit, le bord gauche est lustré (fig. $8, \mathrm{n}^{\circ} 6$ ) ; une chute de burin en silex tertiaire; un burin alterne (un burin d'angle en partie proximale du bord droit et un sur cassure naturelle en partie distale du bord gauche) sur éclat laminaire (fig. 8, no 7 ); un grattoir 
double sur éclat, le front proximal est arrondi et le distal est rectiligne, les retouches sont courtes à longues et abruptes (fig. 8, $\mathrm{n}^{\circ} 8$ ) ; un fragment proximal de lame dont le bord gauche est retouché; un fragment distal d'éclat laminaire (fig. 8, $\mathrm{n}^{\mathrm{o}}$ 9).

\section{UNITÉ STRATIGRAPHIQUE US 42}

Située à l'ouest du même secteur et reconnue à $0,70 \mathrm{~m}$ sous le sol actuel, l'US 42 est constituée par un niveau de limon argileux brun foncé. Identifiée sur 13 m de long, elle pourrait représenter la partie ouest de l'US 31 dont elle a la même nature sédimentaire et contient également des pierres, des graviers, de la céramique et du silex.

L'US 42 a livré cinq fragments de céramique qui correspondent à quatre vases. Le seul tesson diagnostique, un bord, appartient à un vase à lèvre amincie et légèrement éversée qui est comparable aux vases à profil galbé des structures 13, 22 et 75 (fig. 9, $\mathrm{n}^{\mathrm{o}} 1$ ).

Cette structure ne comporte qu'un objet lithique: un éclat laminaire de mise en forme, épais et cortical, le talon est linéaire, le bulbe, peu marqué, porte une lèvre, le plan de frappe a été préparé par abrasion et doucissage (?) (fig. 9, $\mathrm{n}^{\mathrm{o}}$ 2).

\section{LES STRUCTURES FS 47 ET FS 48}

Les structures FS 47 et FS 48 sont des cuvettes; elles sont localisées à l'extrémité ouest de la partie centrale (fig. 2). Creusées dans les limons argileux beiges, elles apparaissent à $0,60 \mathrm{~m}$ sous le sol actuel et sont très similaires : forme circulaire, diamètre d'environ $0,80 \mathrm{~m}$ et profondeur comprise entre $0,15 \mathrm{~m}$ et $0,20 \mathrm{~m}$. Elles sont comblées d'un limon sableux brun foncé gravillonneux. Toutes deux contiennent des pierres et un abondant mobilier archéologique.

\section{Le mobilier de la structure FS 47}

La structure FS 47 a livré deux cent quatre-vingt treize tessons (dont quarante-quatre jointifs) qui appartiennent à au moins dix-sept vases dont treize sont partiellement reconstituables.

Deux vases se distinguent du reste de la céramique par leur aspect grossier, l'épaisseur irrégulière des parois, le lissage sommaire de la surface et le dégraissant hétérométrique: une tasse munie d'une anse en ruban (fig. 10, $\mathrm{n}^{\circ} 1$ ) et un gobelet globulaire qui présente un départ de préhension, quelques millimètres sous le bord (fig. 10, $\mathrm{n}^{\circ} 2$ ). Un
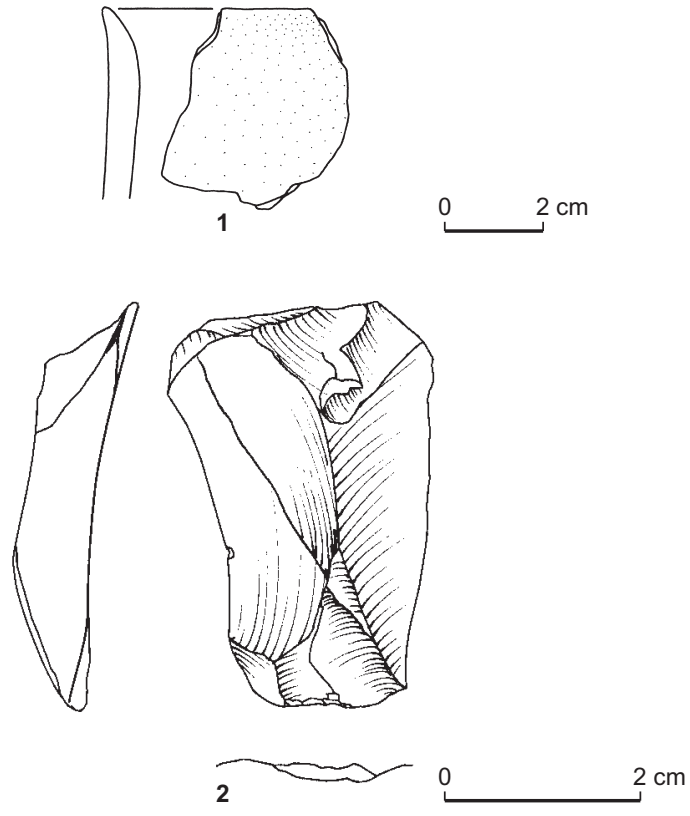

Fig. 9 - Mobilier céramique et industrie lithique de l'unité stratigraphique US 42 (dessin: S. Renault, CNRS et S. Van Willigen, Lampea).

vase sphérique, de taille moyenne à lèvre amincie et à profil légèrement sinueux, est muni d'une barrette multiforée étroite légèrement inclinée (fig. 10, no 3). Quelques tessons permettent de reconstituer partiellement une coupe tronconique à anse en ruban bien dégagée (fig. 10, no 4). Quatre fragments de bord à lèvre amincie et légèrement éversée appartiennent à trois vases différents (fig. 10, $\mathrm{n}^{\text {os }} 5-7$ ). Un petit fragment de coupe tronconique présente la particularité d'avoir une lèvre à section asymétrique, arrondie à l'extérieur et rectiligne à l'intérieur (fig. 10, no 8).

Trois fragments de vases fermés à lèvre amincie et légèrement éversée appartenant à au moins deux vases distincts présentent sur la face extérieure des traces de pigment brun foncé à noir. Le premier est un vase à inflexion basse muni d'une préhension tubulaire horizontale à ensellement apposée à hauteur de l'inflexion. Le décor, qui semble être limité à la partie supérieure du vase, est constitué de deux chevrons emboîtés (fig. 10, no 9). Un fragment de bord appartenant probablement au même vase présente les traces d'un angle et de deux autres lignes parallèles (fig. 10, $\mathrm{n}^{\circ} 10$ ). Bien qu'aucune trace de pigment ne soit visible, trois autres fragments de bord (non figurés) font probablement partie du même récipient. Le deuxième vase est représenté par un fragment de bord avec un départ 

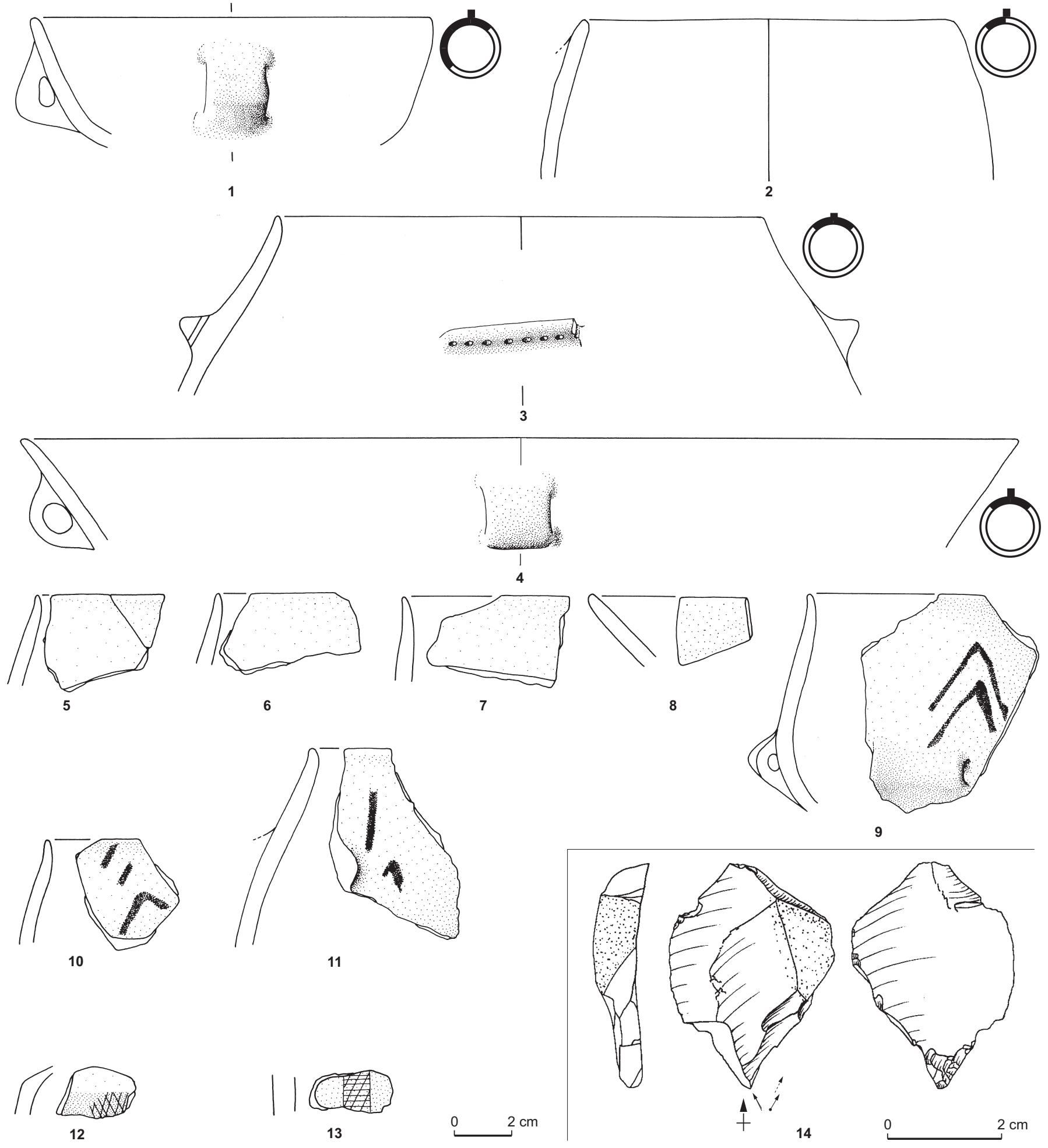

Fig. 10 - Mobilier céramique et industrie lithique de la structure FS 47 (dessin: S. Renault, CNRS et S. Van Willigen, Lampea). 


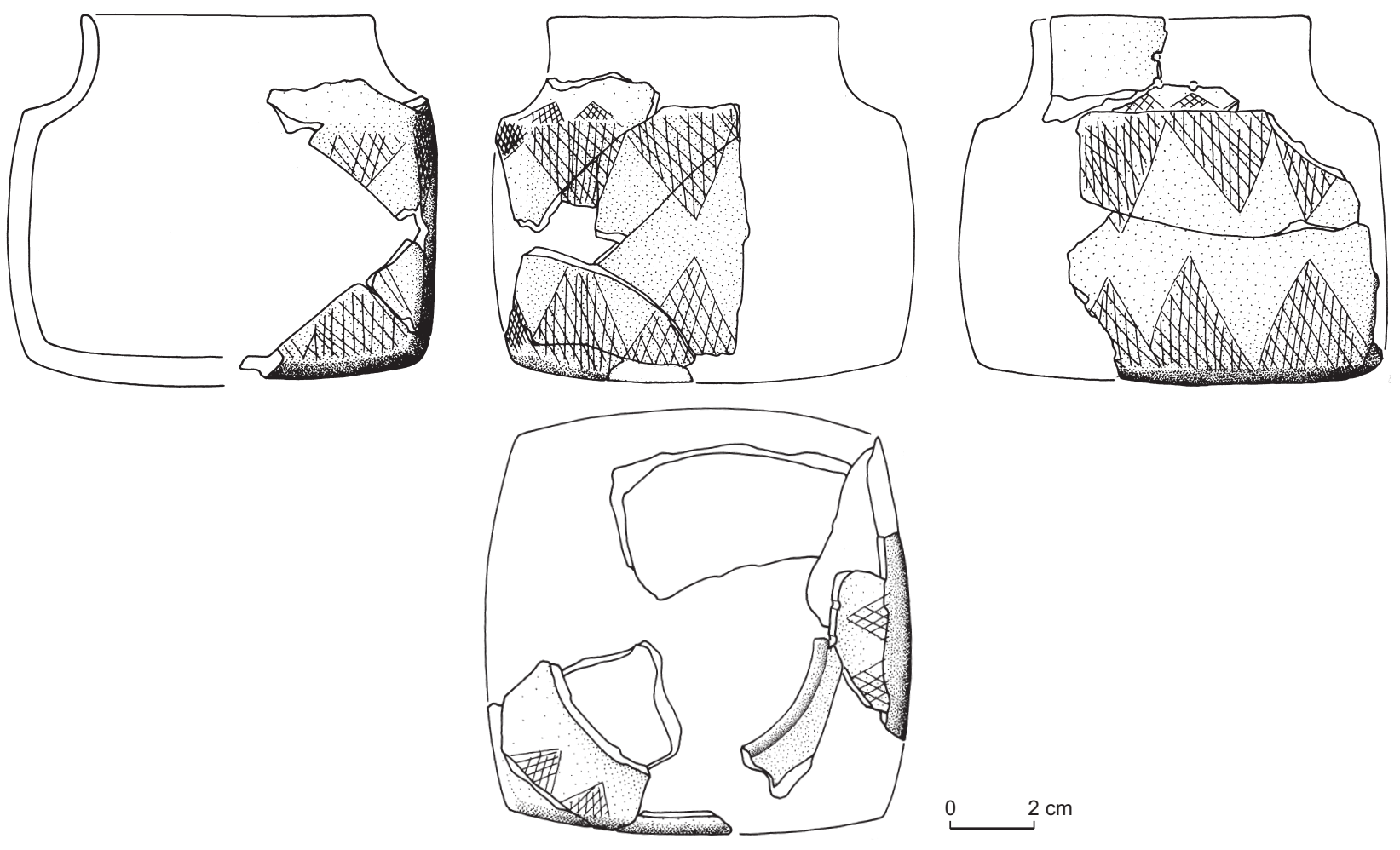

Fig. 11 - Vase à corps cubique $n^{0} 1$ de la structure FS 47 (dessin: S. Van Willigen, Lampea).

de préhension (fig. 10, $\mathrm{n}^{\mathrm{o}}$ 11). À droite de cette dernière, il est possible de distinguer une bande noire subverticale et une ligne brisée.

Seize petits fragments délités et érodés (brûlés ?) sont décorés d'incisions et font probablement partie d'un même vase. Leur mauvais état de conservation ne permet pas d'en restituer la forme. Il est tout au plus possible de dire qu'il présentait des angles (fig. $10, \mathrm{n}^{\circ} 12$ ) et que la surface était décorée d'incisions disposées en bandes quadrillées (fig. 10, $\mathrm{n}^{\mathrm{o}} 13$ ).

Deux séries de tessons permettent de reconstituer deux vases à corps cubique et embouchure circulaire. Le premier, de couleur rouge à brun foncé, n'est que partiellement reconstituable (fig. 11 et fig. $13, \mathrm{n}^{\circ} 1$ ). Le col n'est pas jointif avec le corps du vase et seules trois des quatre faces sont en partie conservées. Le fond et les quatre faces sont légèrement convexes, le col concave présente quatre perforations groupées réalisées avant la cuisson. La lèvre est arrondie et légèrement éversée. Les parois ont une épaisseur relativement constante de l'ordre de $6 \mathrm{~mm}$. Le décor est constitué d'incisions réalisées avant la cuisson. Le motif est le même sur les trois faces conservées: une rangée de triangles quadrillés pointe en bas dans la partie supérieure et une rangée de triangles également quadrillés, pointe en haut, dans la partie inférieure de chacune des faces. Les triangles ayant des dimensions variables, les deux frises ne sont pas symétriques et la zone inornée entre les rangées de triangles présente des contours irréguliers (losanges et zigzags). Le col est également décoré d'une rangée de triangles hachurés plus petits que les précédents qui prennent appui sur l'épaulement qui constitue la base du col. Le second vase à corps cubique, de couleur beige à jaune, a globalement la même forme que le précédent. Il ne s'en différencie que par des dimensions sensiblement plus grandes, un col légèrement éversé ainsi que par son décor conservé sur deux faces (fig. 12 et fig. 13, no 2): deux rangées de triangles quadrillés s'opposent de telle manière qu'ils ménagent une bande inornée en forme de zigzag. Les quelques éléments disponibles pour les deux autres faces permettent de supposer que les quatre faces présentaient le même motif. Un des triangles de la troisième face a cependant la particularité d'être divisé en deux parties par une incision verticale. Sur 

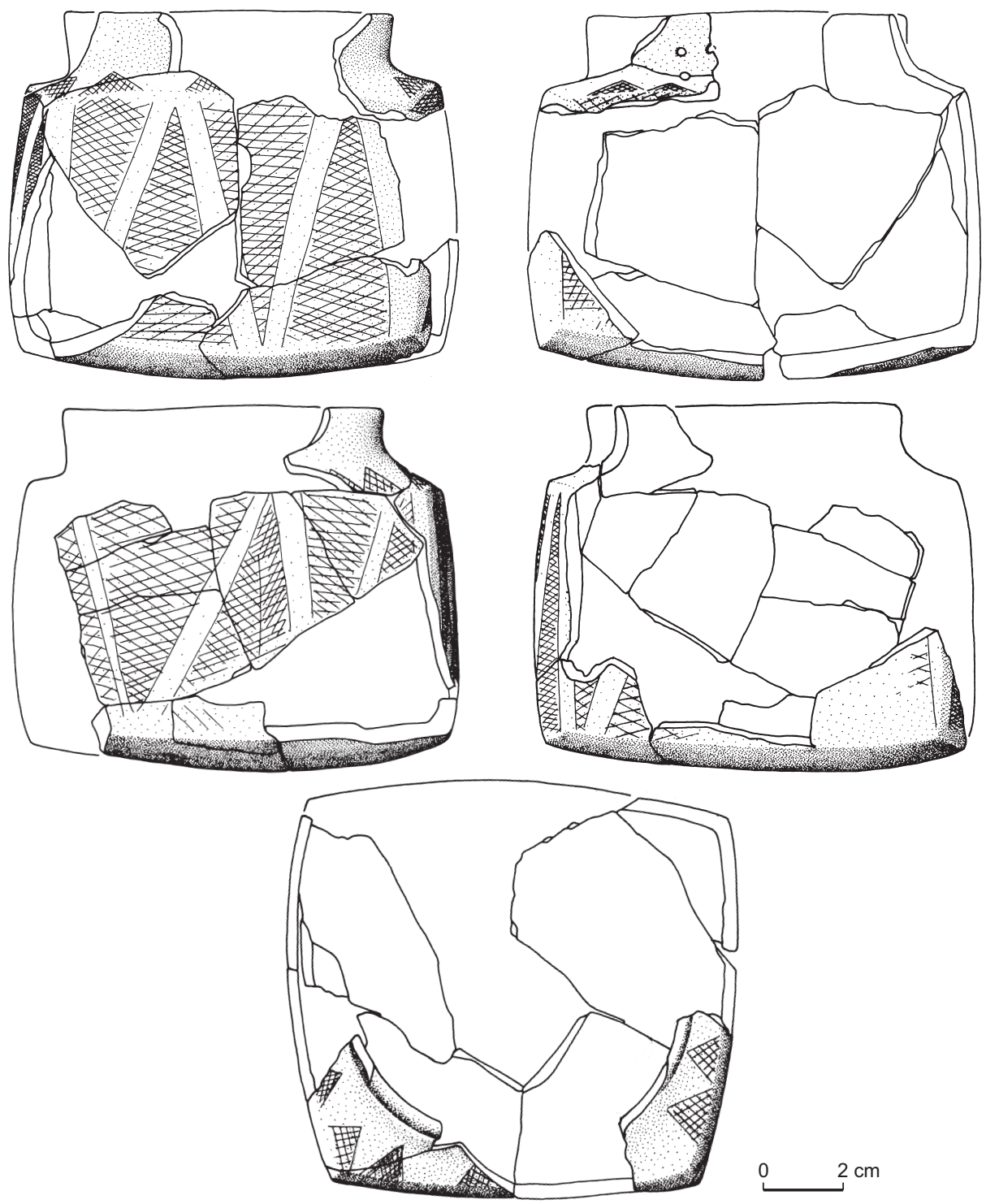

Fig. 12 - Vase à corps cubique $n^{\circ} 2$ de la structure FS 47 (dessin: S. Van Willigen, Lampea).

ces deux vases, les incisions ne présentent aucune trace d'incrustation.

L'industrie lithique qui provient de cet ensemble se limite à un fragment de meule, un percuteur sur galet aplati en grès dont l'arête porte un piquetage de percussion et un burin dièdre sur éclat portant quelques retouches inverses sur le bord droit (fig. 10, $\mathrm{n}^{\mathrm{o}} 14$ ).

Pour finir, la fosse FS 47 a livré vingt-quatre fragments centimétriques d'ossements brûlés dont quatre fragments de crâne humain (enfant), huit fragments d'os longs de faune (les dimensions indiquent qu'il peut s'agir de petits ruminants) et douze fragments indéterminés.

\section{Le mobilier de la structure FS 48}

La structure FS 48 a livré un total de deux cent vingtcinq fragments (dont cent un jointifs) appartenant à un minimum de huit vases dont six sont reconstituables.

Seuls deux fragments diagnostiques n'ont pas pu faire l'objet de remontage: un fragment de vase de forme indé- 

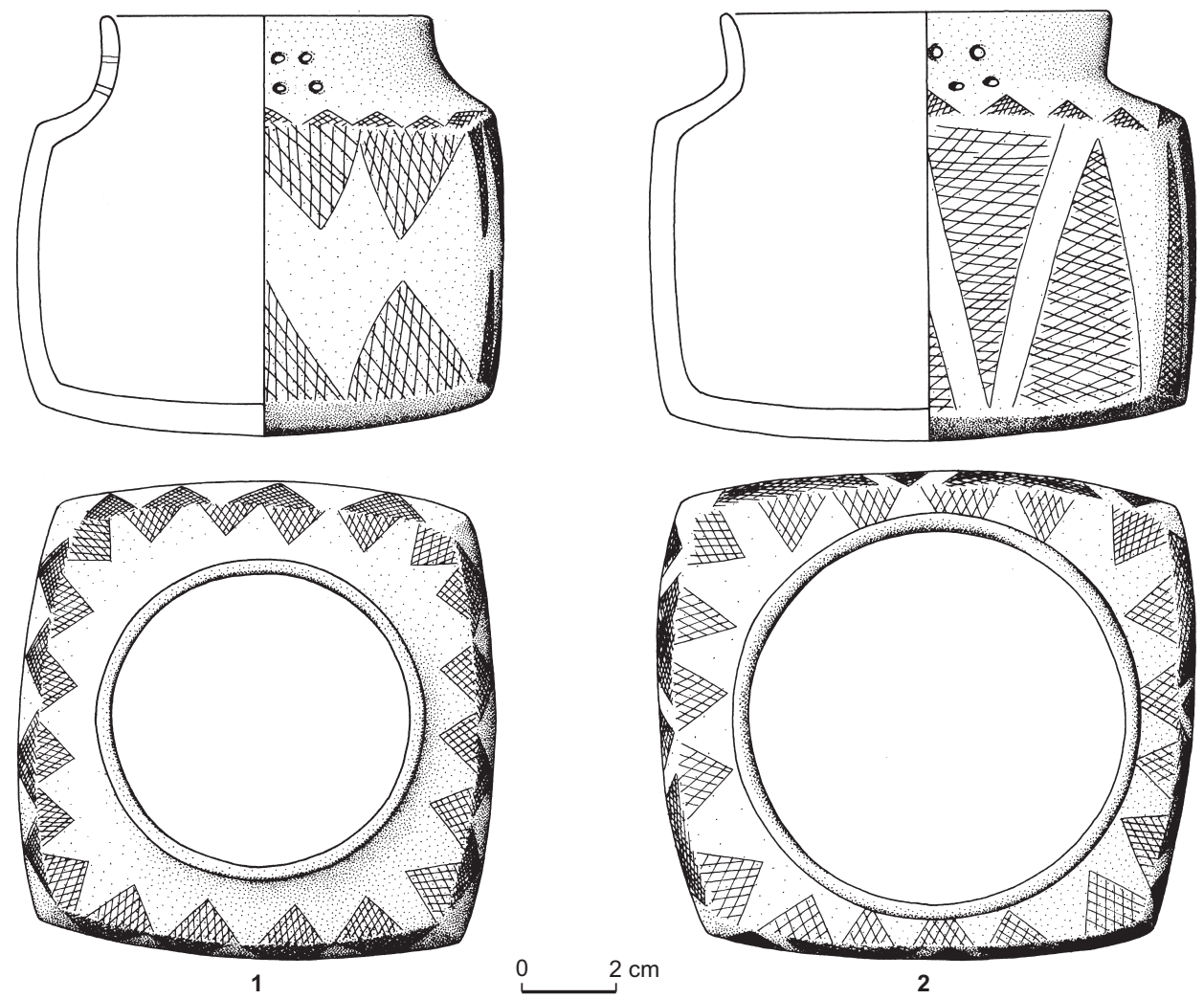

Fig. 13 - Reconstitution graphique des vases à corps cubique $n^{0} 1$ et 2 de la structure FS 47 (dessin: S. Van Willigen, Lampea).

terminée à bord droit et lèvre amincie (fig. 14, $\mathrm{n}^{\circ} 1$ ) et un tesson d'un socle ou d'un vase à corps cubique qui porte un décor incisé (bande ou triangle quadrillé; fig. 14, $\mathrm{n}^{\circ} 2$ ). Une coupe en calotte à lèvre amincie légèrement éversée a pu être partiellement reconstituée (fig. 14, $\mathrm{n}^{\mathrm{o}} 3$ ). Une deuxième coupe en calotte a pu être remontée presque intégralement (fig. 14, $\mathrm{n}^{\circ} 4$ ). Elle a un bord rectiligne et une lèvre arrondie et est munie de deux anses réparties de manière asymétrique puisqu'elles sont situées toutes deux d'un côté de la coupe.

Environ trente-cinq tessons font partie d'une assiette à marli et aile subhorizontale étroite et convexe, munie de deux anses en ruban réparties de manière asymétrique (fig. 15 et 16). Elle présente un décor de triangles quadrillés gravés à sec avant cuisson sur le marli. Un autre décor, à peine visible, est constitué de légères incisions réalisées sur la pâte encore humide à l'extérieur du vase et disposées en forme d'étoile à sept pointes. Ce décor est interrompu au niveau des anses.
Trois vases ont pu être remontés presque totalement. Leur décor est constitué de bandes brunes à noires appliquées sur un fond beige. Le premier est un vase de taille moyenne à fond convexe, carène basse et col fermé légèrement concave (fig. 17, $\mathrm{n}^{\mathrm{o}} 1$ ). Il est muni d'une seule préhension, une anse tunneliforme horizontale placée sur la carène. Le décor est constitué de bandes disposées en chevrons superposés. Le deuxième vase est également de dimensions moyennes, avec un fond convexe, une carène basse et un col rectiligne légèrement fermé (fig. 17, nº 2). Il est muni, au niveau de la carène, d'une seule anse tunneliforme horizontale qui présente un ensellement marqué. Le décor peint est constitué d'une série de chevrons doubles et trois lignes convergentes à gauche de la préhension. Le pigment étant très érodé sur le reste du vase, il est impossible de dire s'il s'agit là d'une irrégularité dans la succession des chevrons ou d'un agencement volontaire. Le troisième vase se distingue des précédents par son profil subsphérique et par son anse tubulaire formée d'une plaquette 

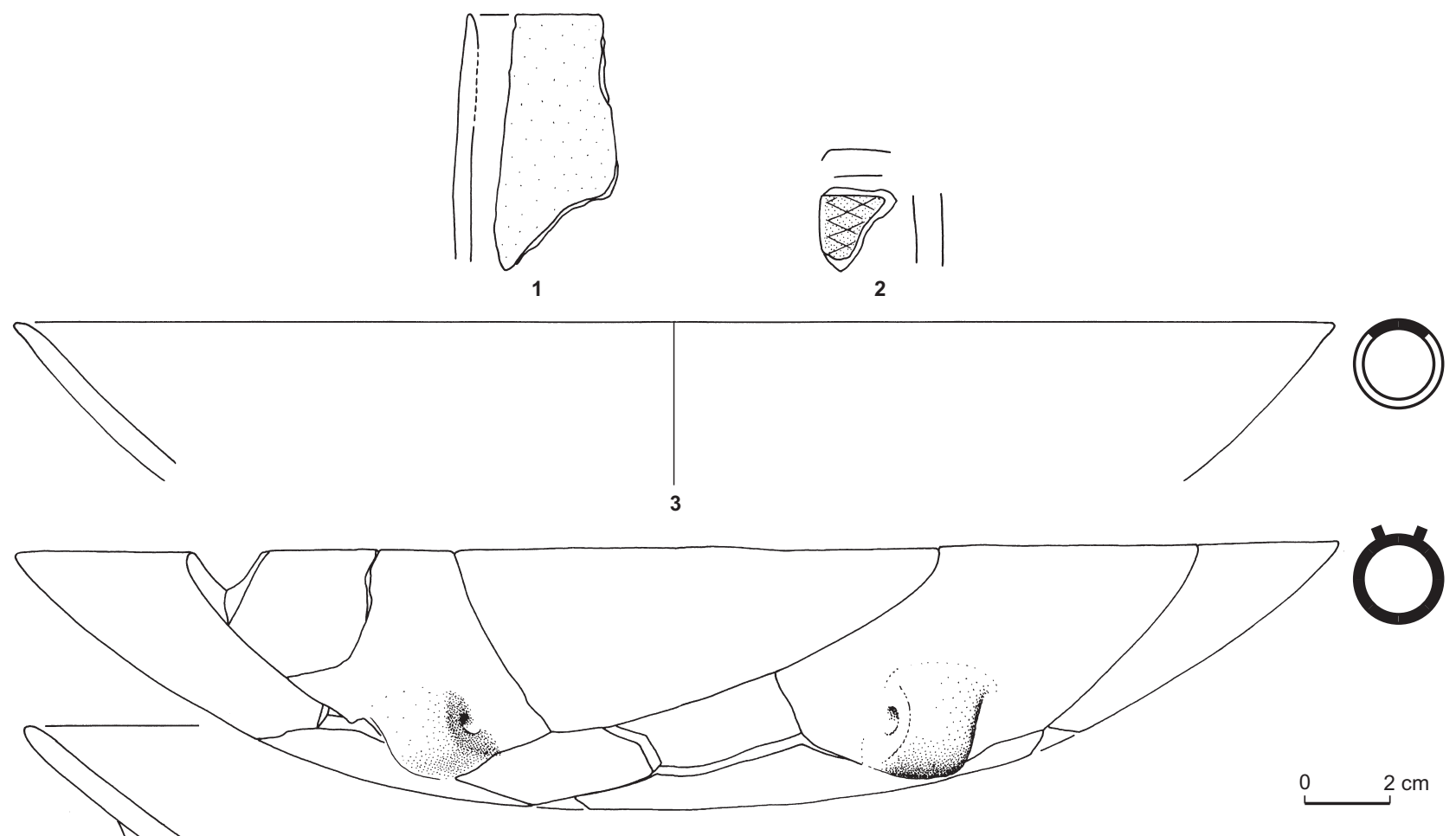

Fig. 14 - Mobilier céramique de la structure FS 48 (dessin: S. Van Willigen, Lampea).

d'argile collée sur la panse dans sa partie inférieure et intégrée, dans sa partie supérieure, entre les deux plaques qui forment le fond et le bord du vase (fig. 17, no $3 a$ et b). Le raccord entre ces deux plaques correspond au plan de cassure horizontal visible dans le prolongement de la partie supérieure de l'anse. Le décor peint, très érodé, n'est conservé que sur un tesson (fig. 17, no 3b). Il est constitué de chevrons superposés qui forment un motif comparable à celui du premier vase.

Dans ces trois cas, le pigment a été appliqué avant la cuisson des vases. En effet, les zones recouvertes par le pigment ont été "protégées» par ce dernier durant la cuisson. Ainsi, aux endroits où le pigment est érodé, elles apparaissent sous forme de bandes claires par rapport aux zones directement exposées au feu qui sont, elles, plus foncées (fig. 18a et b). Ce point écarte toute technique de décor faisant appel à des applications postérieures à la cuisson de pigments ou d'adhésifs tels que le brai de bouleau. Dans le but de déter- miner la nature du pigment utilisé, le décor du vase de la figure 17, 1 a été analysé dans le laboratoire de recherche en conservation du Centre des collections du Musée national suisse. Les méthodes d'analyse utilisées sont non destructives ou microdestructrices lorsque l'échantillon prélevé est de l'ordre de quelques milligrammes. Après un premier examen au microscope, l'analyse des couches colorées est menée directement sur les fragments de céramiques par la spectroscopie de fluorescence $\mathrm{X}$ afin de déterminer les éléments chimiques présents dans les pigments. Ces mêmes échantillons sont ensuite étudiés au spectromètre Raman afin de caractériser les liaisons chimiques présentes dans les pigments et de pouvoir ainsi les identifier.

La spectroscopie infrarouge à transformée de Fourier permet de détecter la présence de restes de produits organiques, tel que le brai de bouleau, qui pourraient être encore présents sur l'échantillon, de l'identifier ou de l'apparenter à une famille chimique. Pour cette dernière 

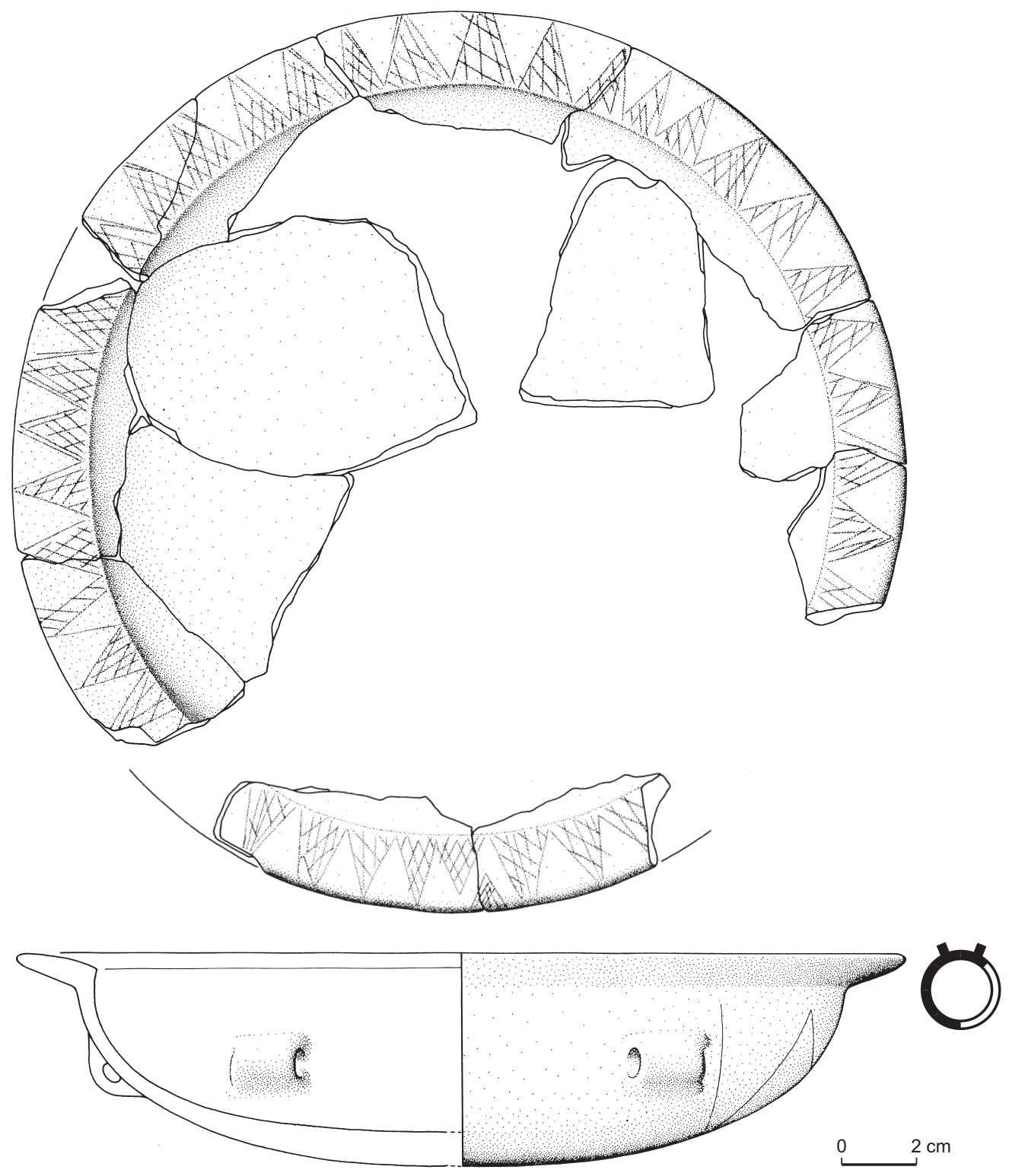

Fig. 15 - Assiette à marli et aile étroite de la structure FS 48 (dessin: S. Van Willigen, Lampea).

analyse, seuls quelques grains de substance prélevés à l'aide d'une aiguille fine sont pressés dans une cellule en diamant afin d'obtenir un film fin de substance qui sera analysé en transmission.

L'examen microscopique a permis de mettre en évidence l'existence d'une couche superficielle à grains fins qui recouvre une pâte à grains hétérogènes, ce qui pourrait indiquer la pose d'un engobe sur le vase. Le pigment a été, quant à lui, appliqué directement sur l'engobe avant la cuisson du vase. La spectroscopie de fluorescence $\mathrm{X}$ a mis en évidence la présence d'un taux de fer plus élevé dans les traits peints que dans l'engobe. La présence d'aucun autre élément (tel que la bauxite) n'a pu être constatée. Il semblerait donc que ce soit cet enrichissement en fer qui donne au décor sa couleur brun foncé. La spectrométrie Raman a permis de mieux caractériser les particules de couleur rouge à rouge foncé présentes dans l'engobe et le pigment qui se sont avérées être de l'oxyde de fer rouge $\left(\mathrm{Fe}_{2} \mathrm{O}_{3}\right)$ de 


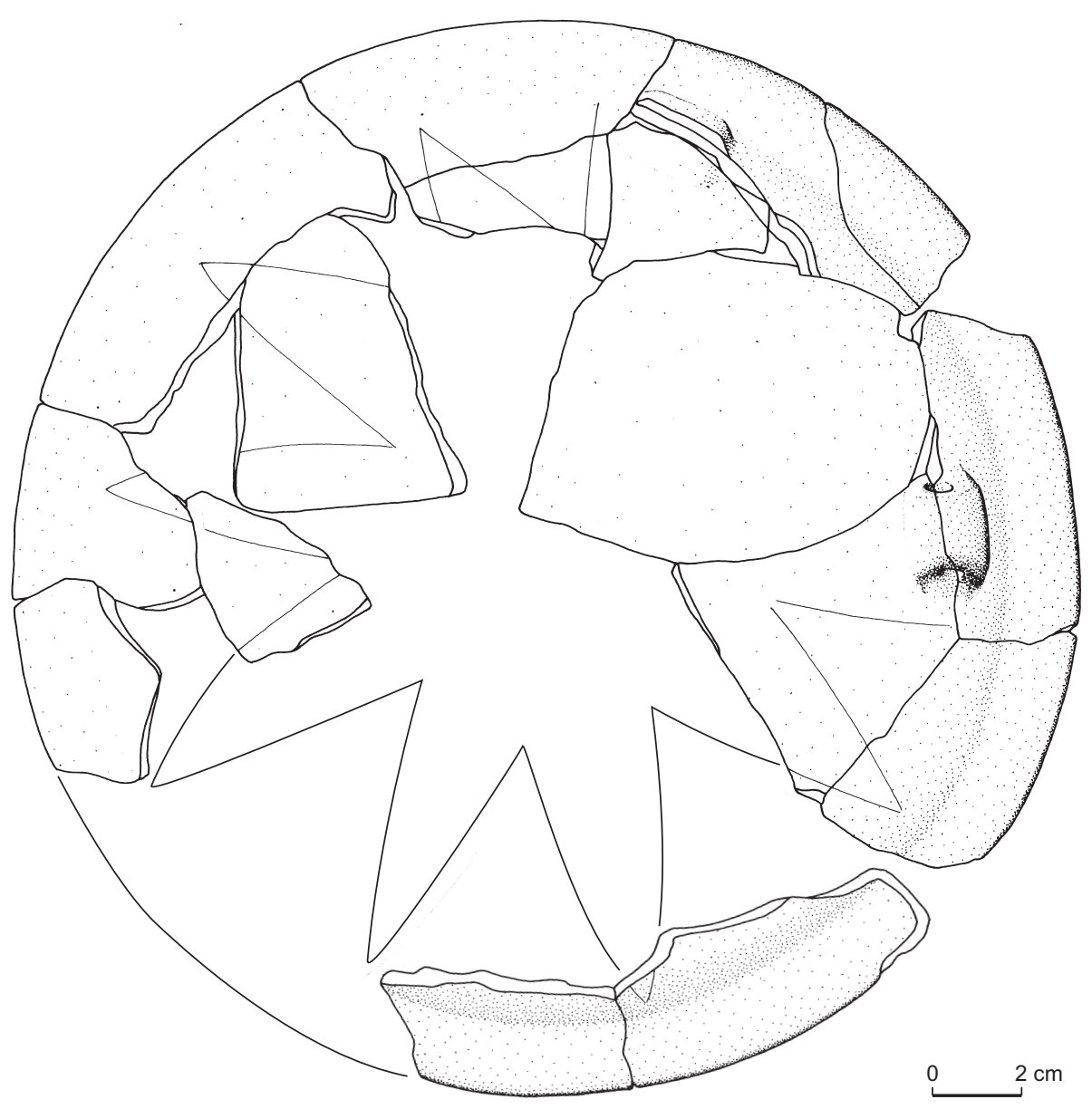

Fig. 16 - Décor externe de l'assiette à marli et aile étroite de la structure FS 48 (dessin: S. Van Willigen, Lampea).

type hématite (fig. 19). On peut supposer que la couleur brune foncée du pigment serait le résultat d'une plus forte concentration en oxyde de fer. La spectrométrie infrarouge à transformée de Fourier montre, quant à elle, que le pigment ne contient actuellement aucune substance organique mais un oxyde métallique anorganique qui peut être de l'oxyde de fer rouge $\left(\mathrm{Fe}_{2} \mathrm{O}_{3}\right)$ de type hématite. Cependant, on ne peut exclure qu'une telle substance organique ait disparu, suite au séjour prolongé du vase dans le sol.

La structure FS 48 n'a livré ni industrie lithique, ni outillage sur matière dure animale. Les restes osseux sont constitués de quatorze fragments brûlés centimétriques parmi lesquels deux fragments de crâne humain (adulte), trois fragments d'os longs humains probables, six fragments de faune (petits ruminants) ainsi que trois fragments de faune indéterminée.
Précisons que les conditions de l'intervention n'ont pas permis une bonne analyse de l'organisation des dépôts dans ces deux structures; il est donc difficile de savoir s'il s'agit de fosses dépotoirs contenant quelques restes humains isolés et brûlés (quatre fragments de crâne d'enfant en FS 47, deux fragments de crâne et trois de diaphyses humaines en FS 48), ou s'il s'agit de restes de sépultures à incinération.

\section{UNITÉ STRATIGRAPHIQUE US 62}

L'US 62 est un niveau limoneux contenant du mobilier archéologique. Il apparaît dans la partie sud du site à une profondeur d'environ $0,70 \mathrm{~m}$ (fig. 2).

Les quatorze fragments de céramique qui proviennent de cet ensemble correspondent à treize vases différents. Parmi eux, il est possible de dénombrer quatre tessons 

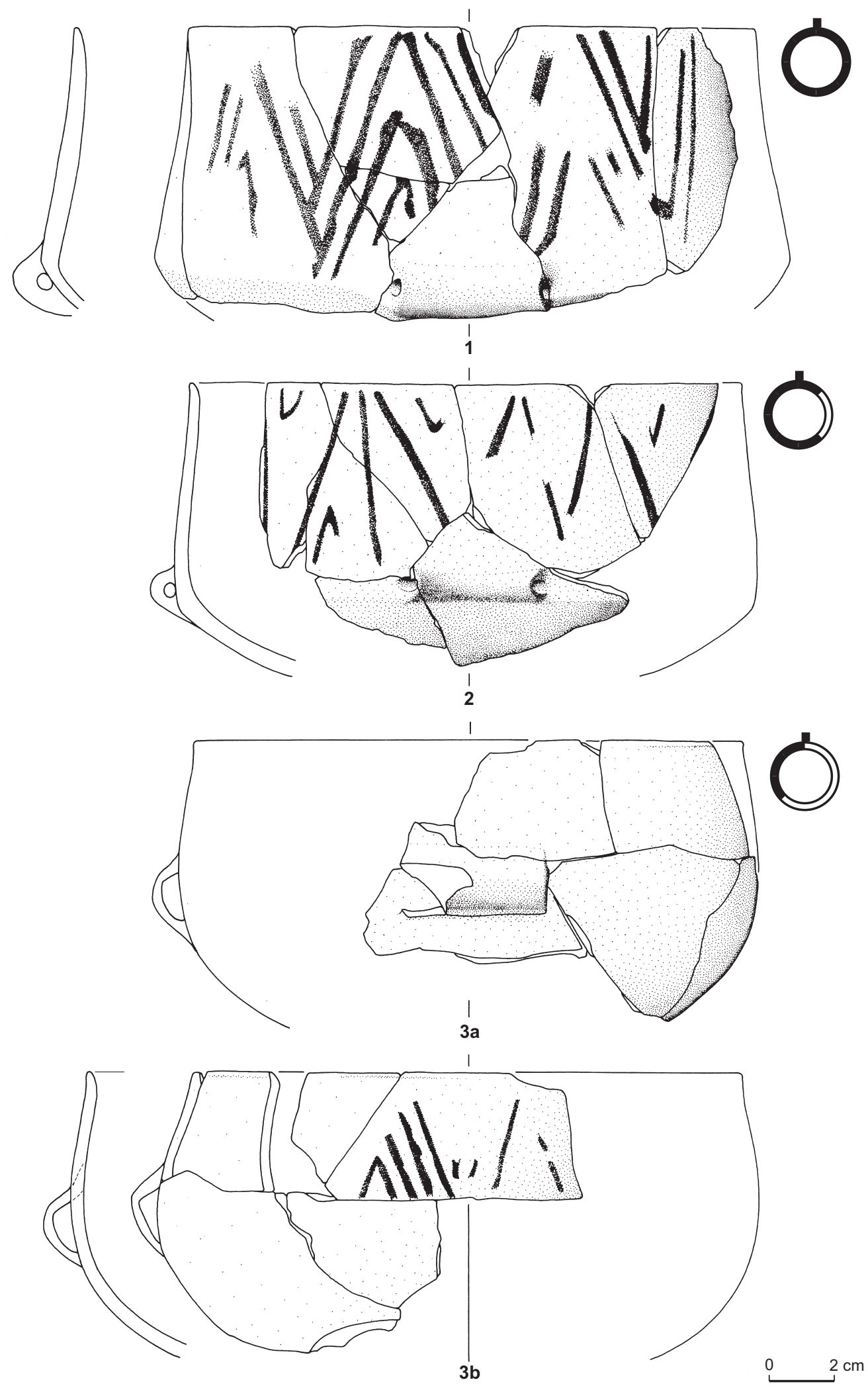

Fig. 17 - Les trois vases peints de la structure FS 48 (dessin: S. Van Willigen, Lampea). 

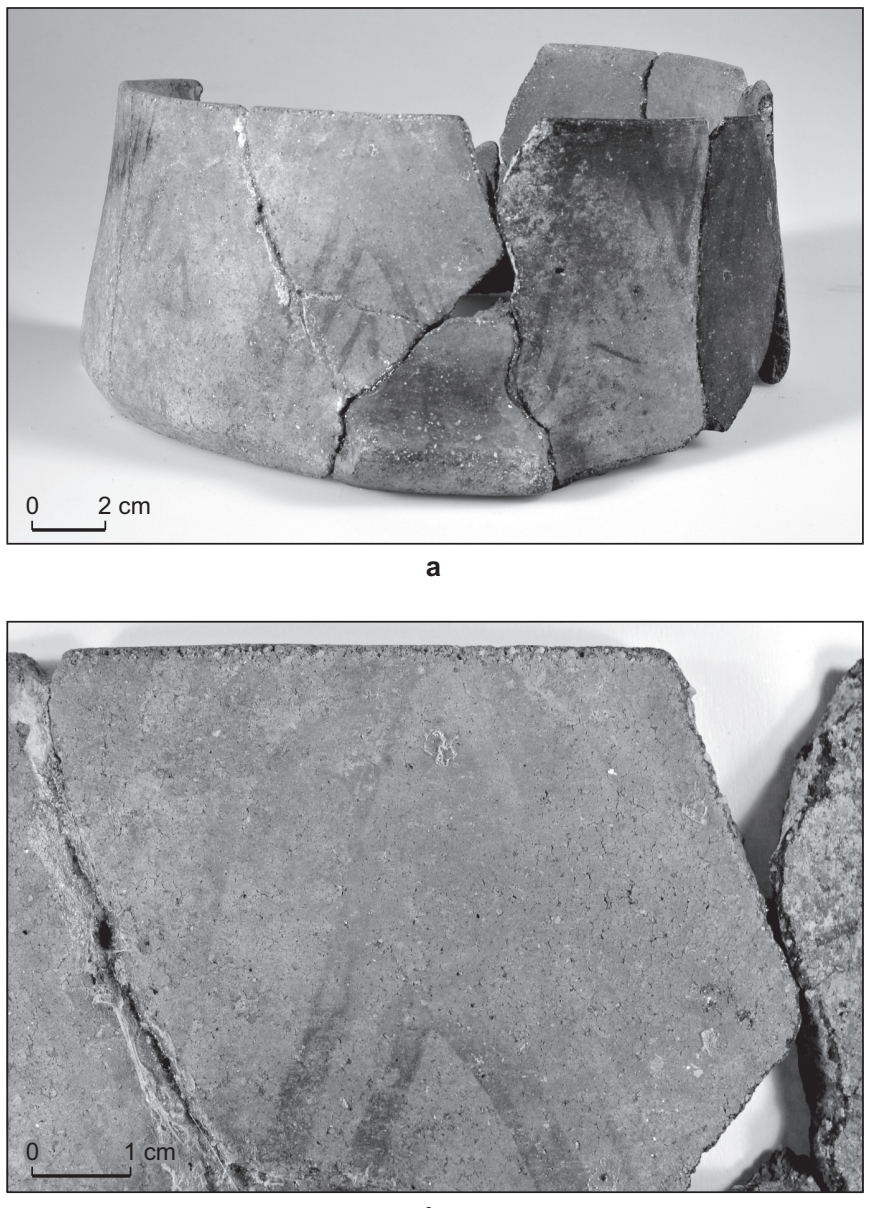

b

Fig. 18 - Photographie et détail du vase peint $n^{0} 1$ de la structure FS 48 (cliché: C. Cevey, Musée national suisse).

diagnostiques dont deux bords, une préhension et un épaulement. Le premier bord appartient à une coupe tronconique de grandes dimensions (fig. 20, $\mathrm{n}^{\circ} 1$ ). Le deuxième est un bord rectiligne à lèvre légèrement aplatie (fig. 20, $\left.n^{\circ} 2\right)$. Un fragment de panse correspond à un petit vase fermé à épaulement doux et col fermé légèrement convexe (fig. 20, no 3). L'épaulement est épaissi et perforé verticalement au niveau de la cassure. Pour finir, un fragment de panse porte un cordon multiforé d'un type particulier puisqu'il présente, vu en coupe, un resserrement au niveau des perforations (fig. 20, $\mathrm{n}^{\circ} 4$ ).

L'industrie lithique comporte vingt-cinq objets: un nucléus cortical à éclats, patiné brûlé; dix éclats dont trois laminaires; un burin sur cassure, sur un fragment proximal de lame à nervures rectilignes et section trapézoïdale irrégulière dont le plan de frappe a été préparé par abrasion et doucissage, la face inférieure porte une lèvre et des ondu-

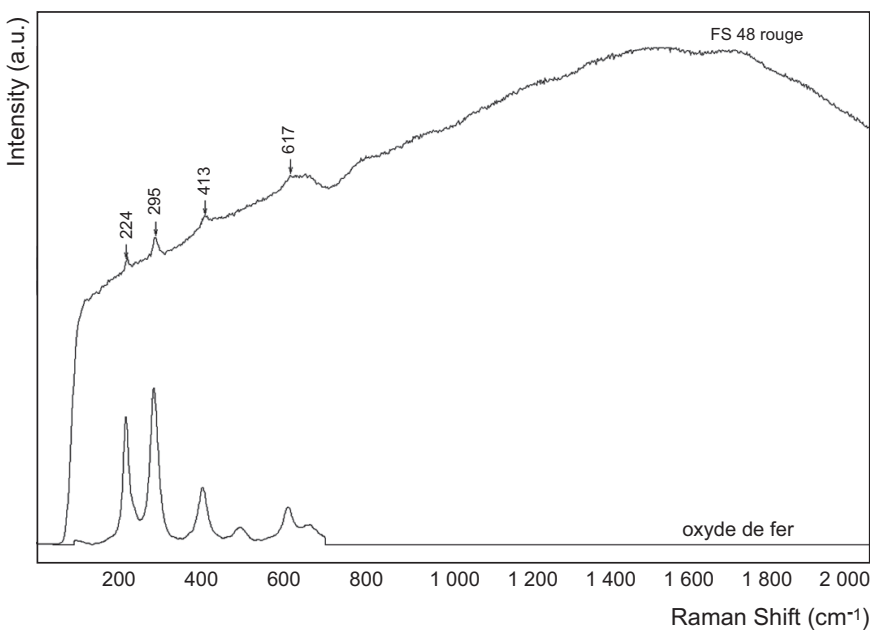

Fig. 19 - Spectre Raman des particules rouges (en haut) et spectre de référence de l'oxyde de fer de type hématite (en bas) du vase peint $n^{0} 1$ de la FS 48 (DAO: K. Hunger, Musée national suisse).

lations (fig. 20, $\mathrm{n}^{\circ} 5$ ); un fragment brûlé de lame à crête (fig. 20, $n^{\circ} 6$ ); un fragment mésial de petite lame patinée à section trapézoïdale asymétrique et nervures rectilignes (code de débitage 2-1-2') et portant quelques retouches partielles sur les deux bords (fig. 20, $\mathrm{n}^{\mathrm{o}} 7$ ); un fragment mésial de lame à quatre versants, bords et nervures rectilignes et relativement parallèles (fig. $20, \mathrm{n}^{\circ} 8$ ) ; un fragment mésial de petite lame porte des retouches abruptes sur le bord gauche (fig. 20, $\mathrm{n}^{\circ} 9$ ); deux éclats laminaires corticaux portent des retouches partielles sur le bord gauche (fig. 20, $\mathrm{n}^{\text {os }} 10$ et 11); un grattoir à front rectiligne (troncature ?) sur éclat cortical obtenu par retouche semi-abrupte et longue (fig. 20, $\mathrm{n}^{\circ} 12$ ); l'extrémité distale présente les traces d'au moins trois enlèvements de coup de burin (bord gauche); un fragment proximal de lame à quatre versants à la corniche abrasée (fig. 20, no 13); un perçoir dégagé par retouche abrupte à semi-abrupte courte à longue sur éclat cortical épais (fig. 20, $\mathrm{n}^{\mathrm{o}} 14$ ); un éclat épais comporte un coup de burin et un réaménagement du versant gauche par des retouches directes profondes puis un second enlèvement de coup de burin sur le versant droit (burin dièdre ?) (fig. 20, $\mathrm{n}^{\circ} 15$ ); un fragment distal brûlé de lame, de section triangulaire, dont la nervure et le bord gauche sont rectilignes et parallèles, le bord droit portant une retouche courte et abrupte (fig. 20, $\mathrm{n}^{\circ} 16$ ); un fragment de lame appointée en silex zoné tertiaire, à section trapézoïdale et à nervures rectilignes et parallèles comporte des retouches directes et abruptes (fig. 20, $\mathrm{n}^{\circ} 17$; code de débitage 2-1-2'). 

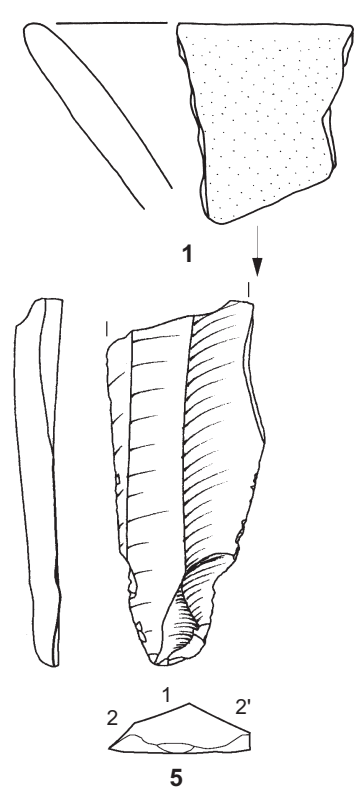
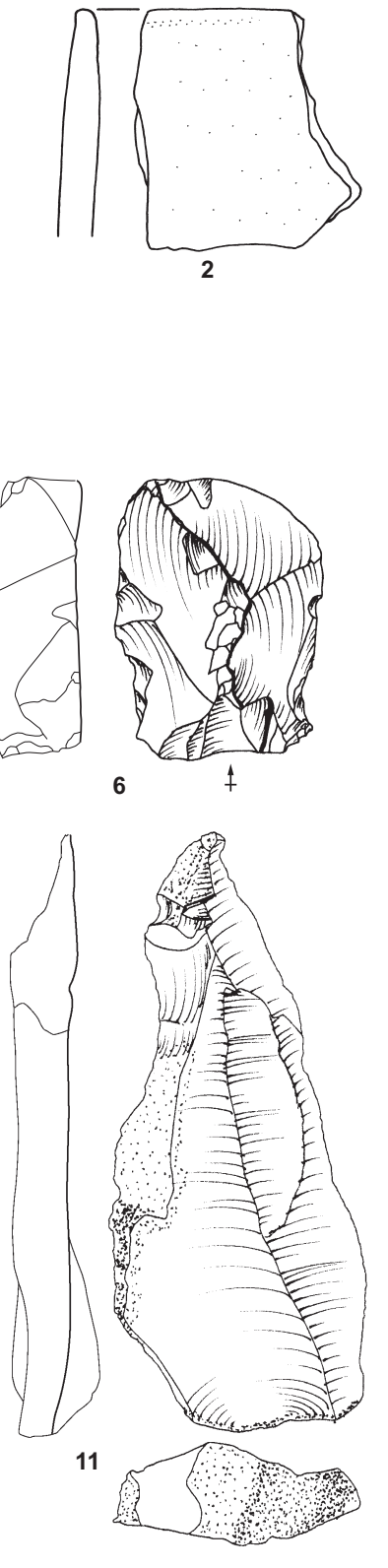
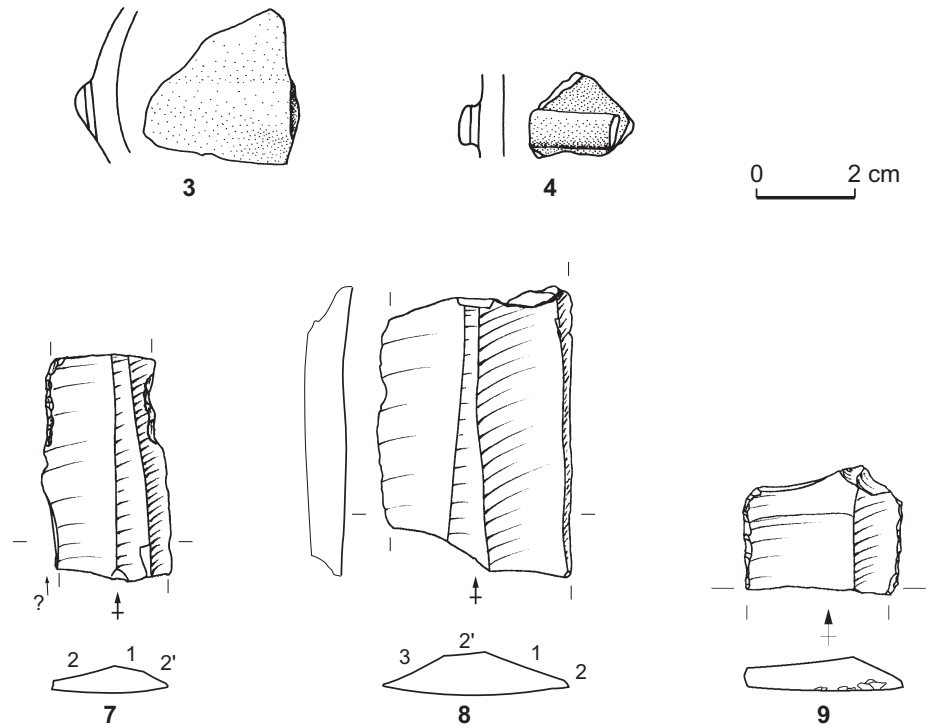
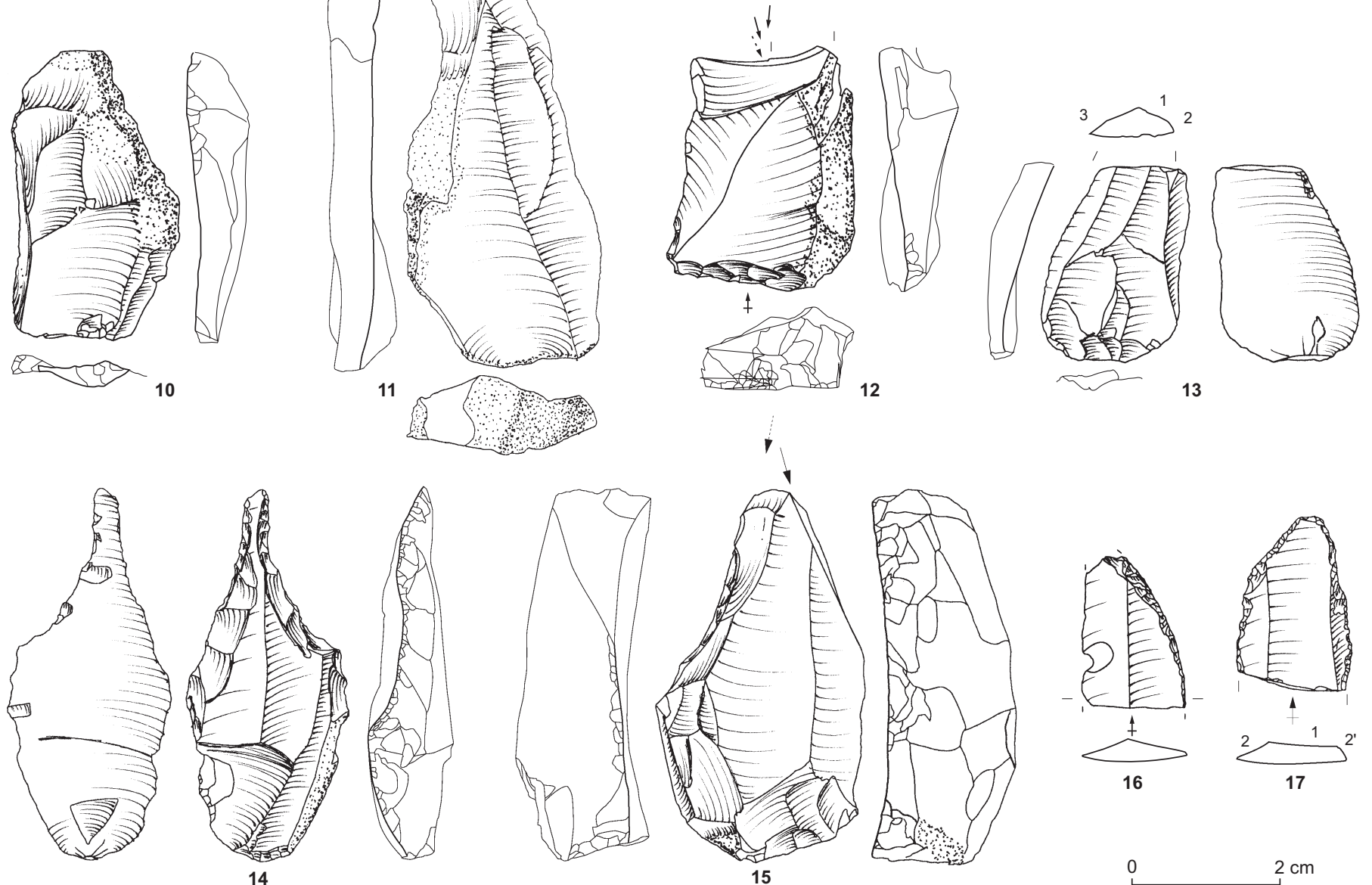

Fig. 20 - Mobilier céramique et industrie lithique de l'unité stratigraphique US 62 (dessin: S. Renault, CNRS, et S. Van Willigen, Lampea). 

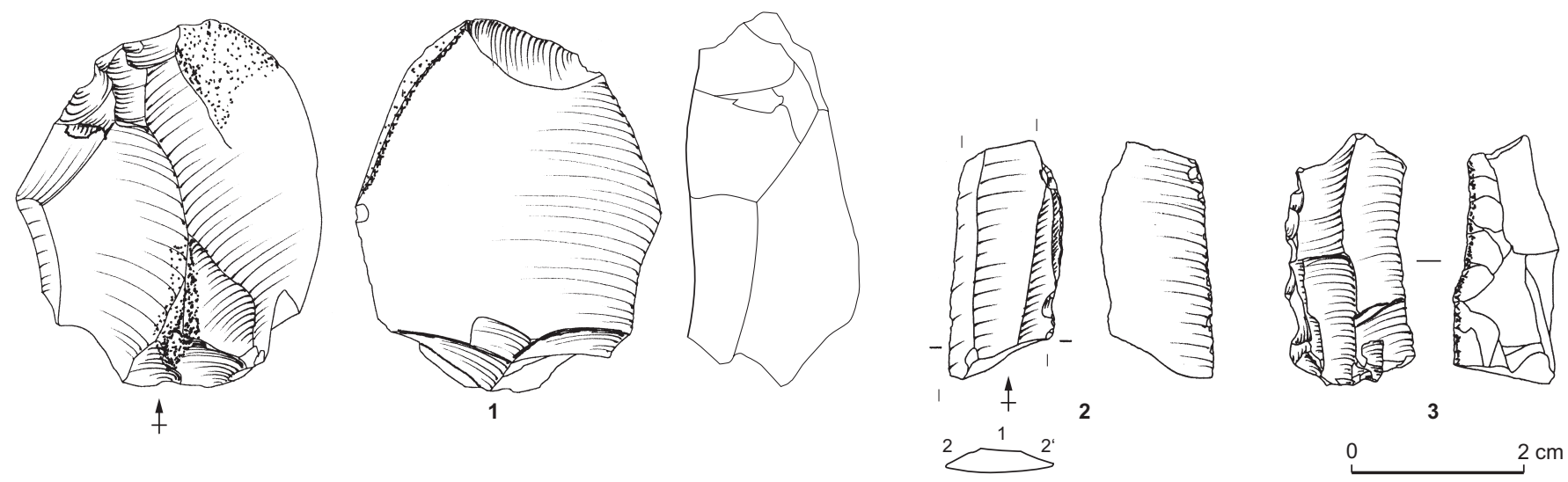

Fig. 21 - Industrie lithique de l'unité stratigraphique US 67 (dessin: S. Renault, CNRS).

\section{UNITÉ STRATIGRAPHIQUE US 67}

L'US 67 caractérise le mobilier trouvé hors des structures dans la tranchée de sondage TR 46. Elle est localisée dans la partie sud du site et apparaît à $0,85 \mathrm{~m}$ de profondeur; c'est un niveau limoneux contenant du mobilier archéologique.

Hormis quelques fragments de céramique non diagnostiques, cette unité stratigraphique a livré neuf objets lithiques: un débris thermique; un fragment de nucléus cortical à éclats; cinq éclats dont deux en silex blond bédoulien, un en silex gris et deux patinés (fig. 21, $\mathrm{n}^{\mathrm{o}} 1$ ) ; un nucléus cortical à lamelles (sur éclat) à plans de frappe opposés ou un burin à deux enlèvements de burin opposés (la pièce porte des retouches sur un bord pour cintrer la table) (fig. 21, $\mathrm{n}^{\circ} 3$ ); un fragment mésial de lame en silex bédoulien chauffé de section trapézoïdale irrégulière (fig. 21, $\mathrm{n}^{\circ}$ 2) : la face inférieure porte des ondulations, les nervures et le bord gauche sont relativement rectilignes, le bord droit porte des retouches courtes/marginales semiabruptes à obliques en partie distale (code de débitage 2-1-2'), l'écrasement du bord et du méplat pouvant être dû à l'utilisation d'un burin (?).

\section{L'UNITÉ STRATIGRAPHIQUE US 70}

Située dans la partie sud du site, cette structure apparaît à $0,85 \mathrm{~m}$ de profondeur. Il s'agit d'un fond de vase d'un diamètre de $0,30 \mathrm{~m}$, probablement implanté dans une petite fosse creusée dans le substratum argileux. Il contient la partie supérieure d'un autre vase plus petit.
Le fond de vase étant dans un état de conservation extrêmement mauvais, il n'a pas pu être reconstitué. Le deuxième récipient a pu, quant à lui, être partiellement remonté. Il s'agit d'un vase de dimensions moyennes de couleur grise à dégraissant abondant constitué de grains de calcite (fig. 22, $\mathrm{n}^{\circ} 1$ ). Ce vase, de forme tronconique, est muni d'une série de boutons proéminents appliqués sous le bord. Un fragment de panse présentant un départ de préhension (anse en boudin ou gros bouton à section ronde) appartenait vraisemblablement au même vase mais son emplacement n'a pu être déterminé.

L'industrie lithique comporte trois objets en silex bédoulien non chauffé (fig. 22, ${ }^{\text {os }} 2$ à 4 ) : une tablette de ravivage de nucléus porte en partie distale des retouches inverses semi-abruptes à abruptes et à délinéation planoconvexe (grattoir ?) (fig. 22, $\mathrm{n}^{\circ}$ 2); une lamelle torse à section irrégulière (trois et quatre versants) et talon punctiforme (fig. 22, $\mathrm{n}^{\mathrm{o}} 3$ ); une lamelle à section irrégulière (trois et quatre versants) : le plan de frappe a été préparé par doucissage et par quelques enlèvements abrasant la corniche, le talon présente une lèvre, l'angle de chasse est légèrement aigu, le bulbe est diffus et la face inférieure est relativement régulière. La partie non retouchée des deux bords est rectiligne, la retouche, semi-abrupte à abrupte et courte, affectant la moitié distale du bord droit et la partie distale du bord gauche (fig. 22, $\mathrm{n}^{\circ} 4$ ).

\section{STRUCTURE FS 75}

Toujours dans la partie sud du site, la fosse 75 apparaît à $0,60 \mathrm{~m}$ sous le sol actuel. Elle a une forme irrégulière et un 

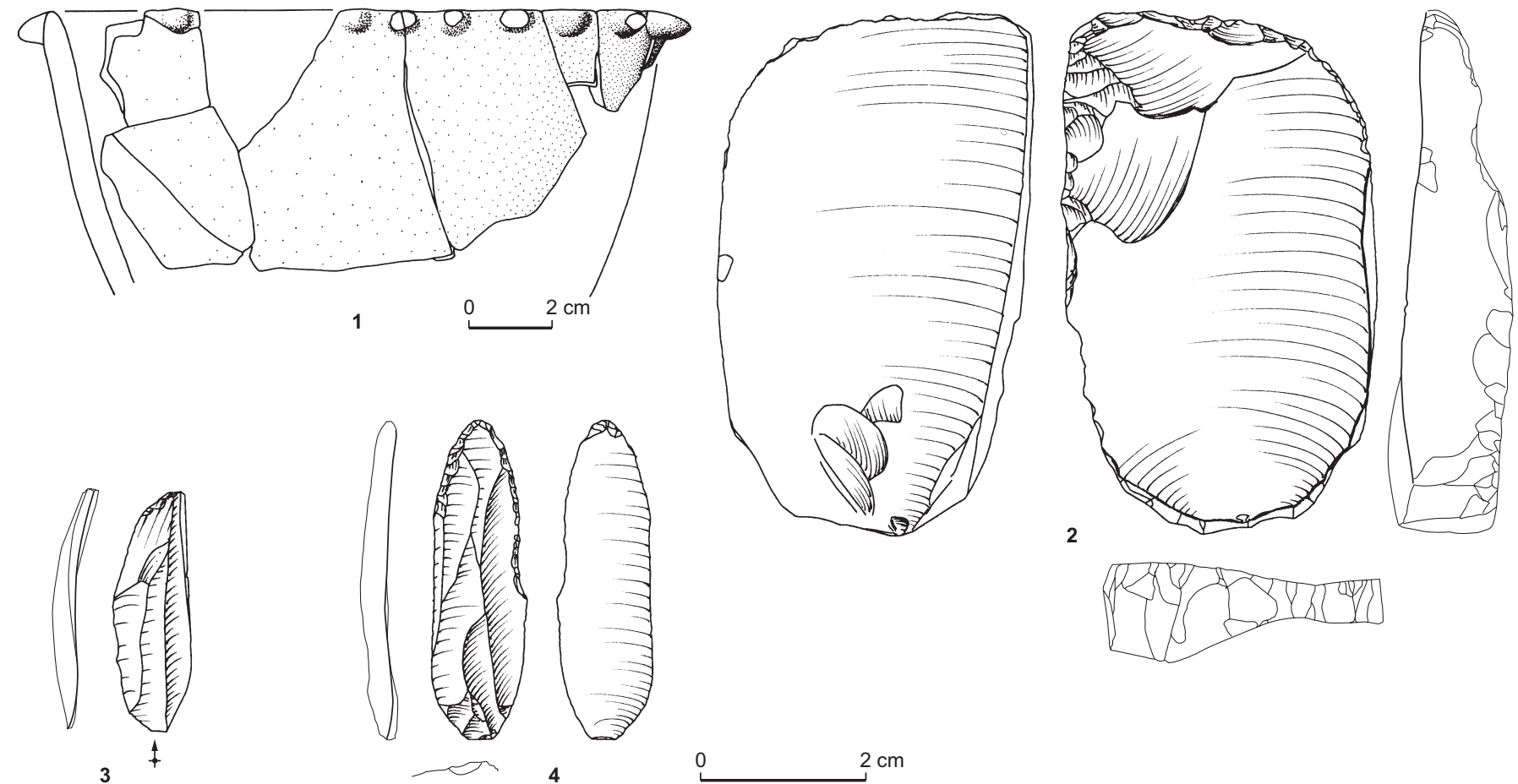

Fig. 22 - Mobilier céramique et industrie lithique de l'unité stratigraphique US 70 (dessin: S. Renault, CNRS et S. Van Willigen, Lampea).

diamètre maximal observé de 1,80 m. Creusée dans l'argile limoneuse vert-jaune, elle a un comblement un peu plus limoneux et gris contenant un abondant mobilier.

La structure FS 75 a livré deux cent soixante et un tessons (dont cinquante-six jointifs) qui appartiennent à au moins dix-sept vases différents. Les soixante-trois tessons diagnostiques permettent de reconstituer au moins partiellement sept vases. Vingt et un tessons diagnostiques n'ont pas pu faire l'objet de remontages.

Deux fragments de bord non jointifs font partie d'une coupe en calotte dont le bord présente un méplat interne délimité vers le bas par un ressaut (fig. 23, no 1). À la différence des assiettes à marli qui présentent une paroi externe à profil concave, voire discontinu, cette coupe a une paroi externe convexe. Un bord correspond à un petit vase tronconique ou à la partie supérieure d'un vase à col (fig. 23, no 2). Deux tessons jointifs appartiennent à un petit vase à bord rectiligne et tendance biconique munis de boutons rectangulaires biforés, répartis sur deux niveaux (fig. 23, $\mathrm{n}^{\circ} 3$ ). Un vase de taille moyenne à inflexion basse et col concave fermé a pu être reconstitué à partir de la quasi-totalité de ses fragments (fig. 23, $n^{\circ} 4$ ). Il présente deux boutons plats perforés horizontalement situés à hauteur de l'inflexion et surmontés par un épaulement limité à l'espace compris entre les deux préhensions (épaulement partiel). Un vase inorné, toujours de taille moyenne, présente une inflexion basse et un col rectiligne vertical (fig. 23, $n^{\circ} 5$ ). Cinq fragments permettent de reconstituer un vase de taille moyenne à inflexion basse et col rectiligne fermé (fig. 24, no 1 ). Il est muni d'un bandeau partiellement multiforé et cannelé. Ces cannelures sont disposées entre les perforations verticales suggérant ainsi des multitubulures (fig. 24, $\mathrm{n}^{\mathrm{o}} 1$ à droite), mais aussi entre les groupes de perforations verticales (fig. 24, $\mathrm{n}^{\mathrm{o}} 1 \mathrm{au}$ milieu). Deux tessons jointifs appartiennent à la base d'une assiette à socle conique qui présente un décor gravé avant cuisson de bandes quadrillées organisées en chevrons (fig. 24, no 2). Ces deux tessons montrent encore par endroits les restes d'une matière blanche qui recouvrait les gravures. Après un examen préalable au microscope, deux analyses ont été réalisées dans le but de caractériser ce matériau: spectroscopie de fluorescence X et spectrométrie infrarouge à transformée de Fourier. Les deux tessons jointifs sont de couleurs différentes. Le plus grand des deux est noir alors que le plus petit est rouge brique. C'est justement sur ce dernier que l'incrustation est la mieux conservée (fig. 25a et b). Les analyses ont porté 

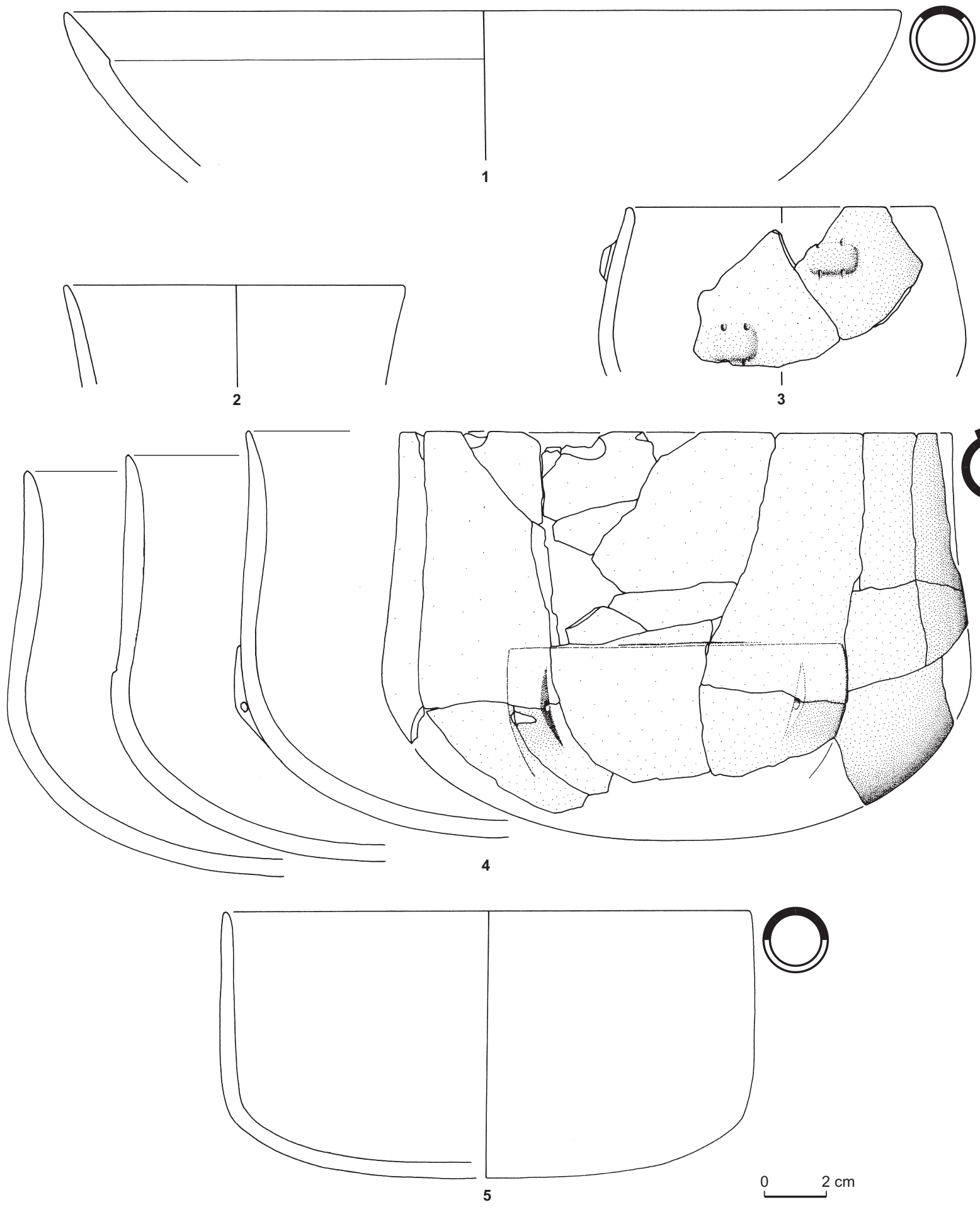

Fig. 23 - Mobilier céramique la structure FS 75 (dessin: S. Van Willigen, Lampea). 

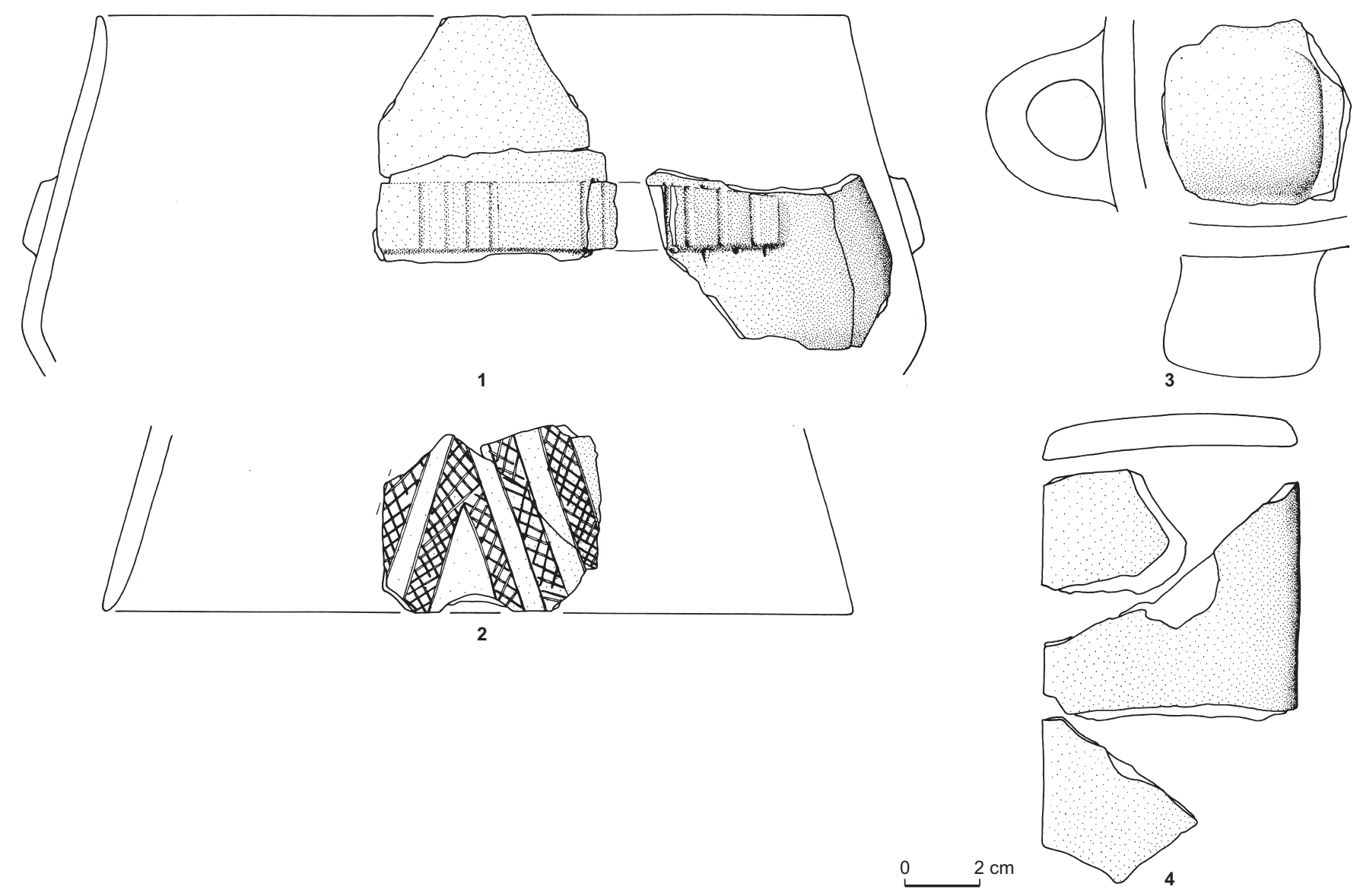

Fig. 24 - Mobilier céramique la structure FS 75 (dessin: S. Van Willigen, Lampea).

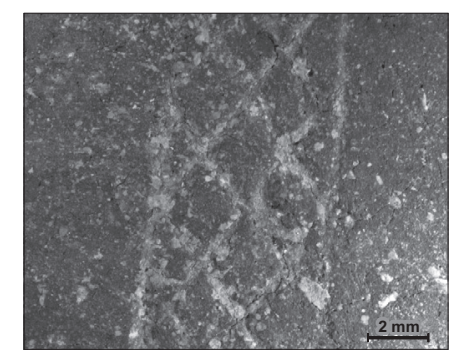

a

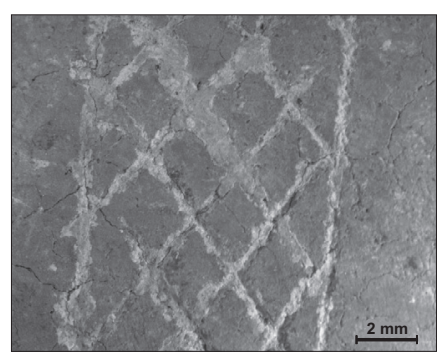

b

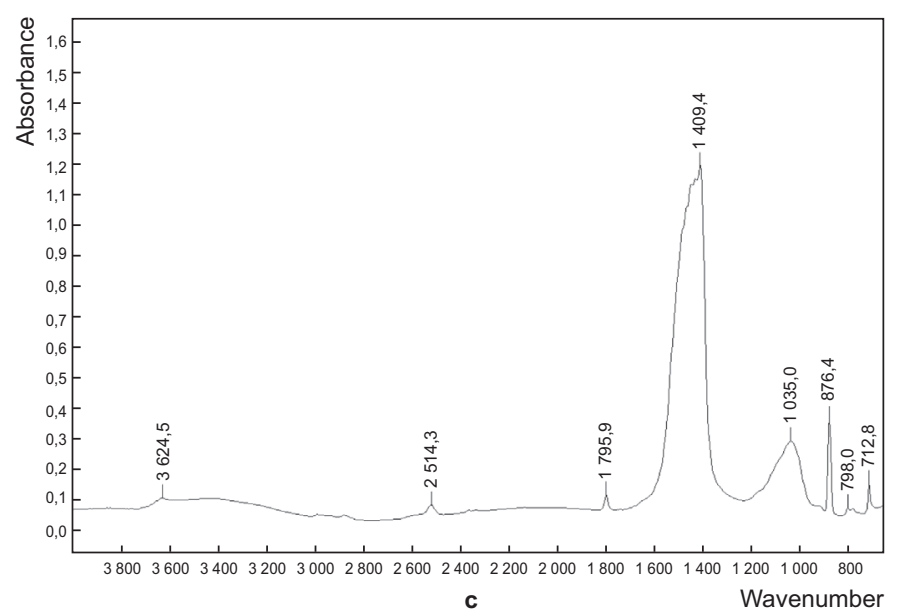

Fig. 25 - Macrophotographie des incrustations sur le tesson noir (a) et rouge (b) de la fig. 24, $n^{\circ} 2$; spectre FTIR du matériau présent dans les incrustations du tesson rouge (c) (DAO: K. Hunger, Musée national suisse). 

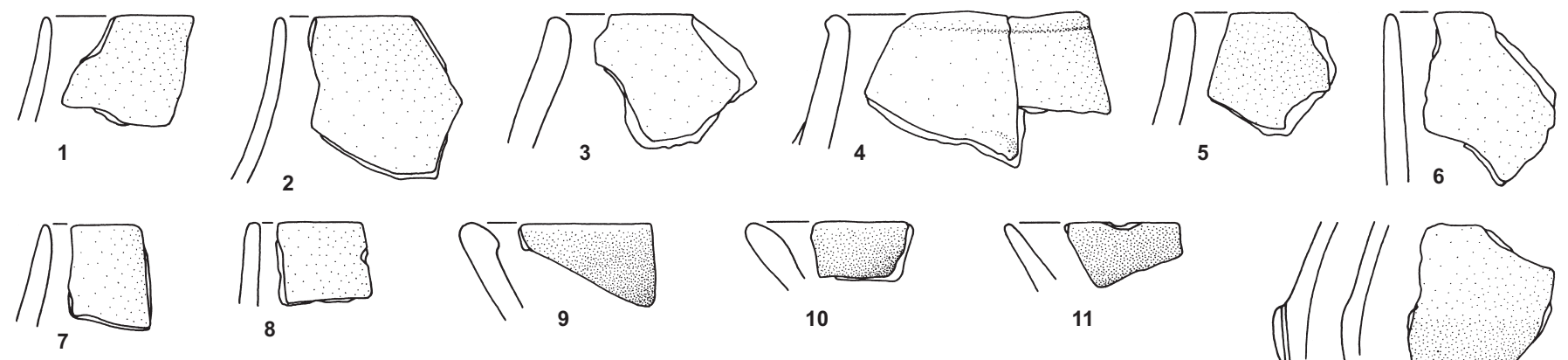

10
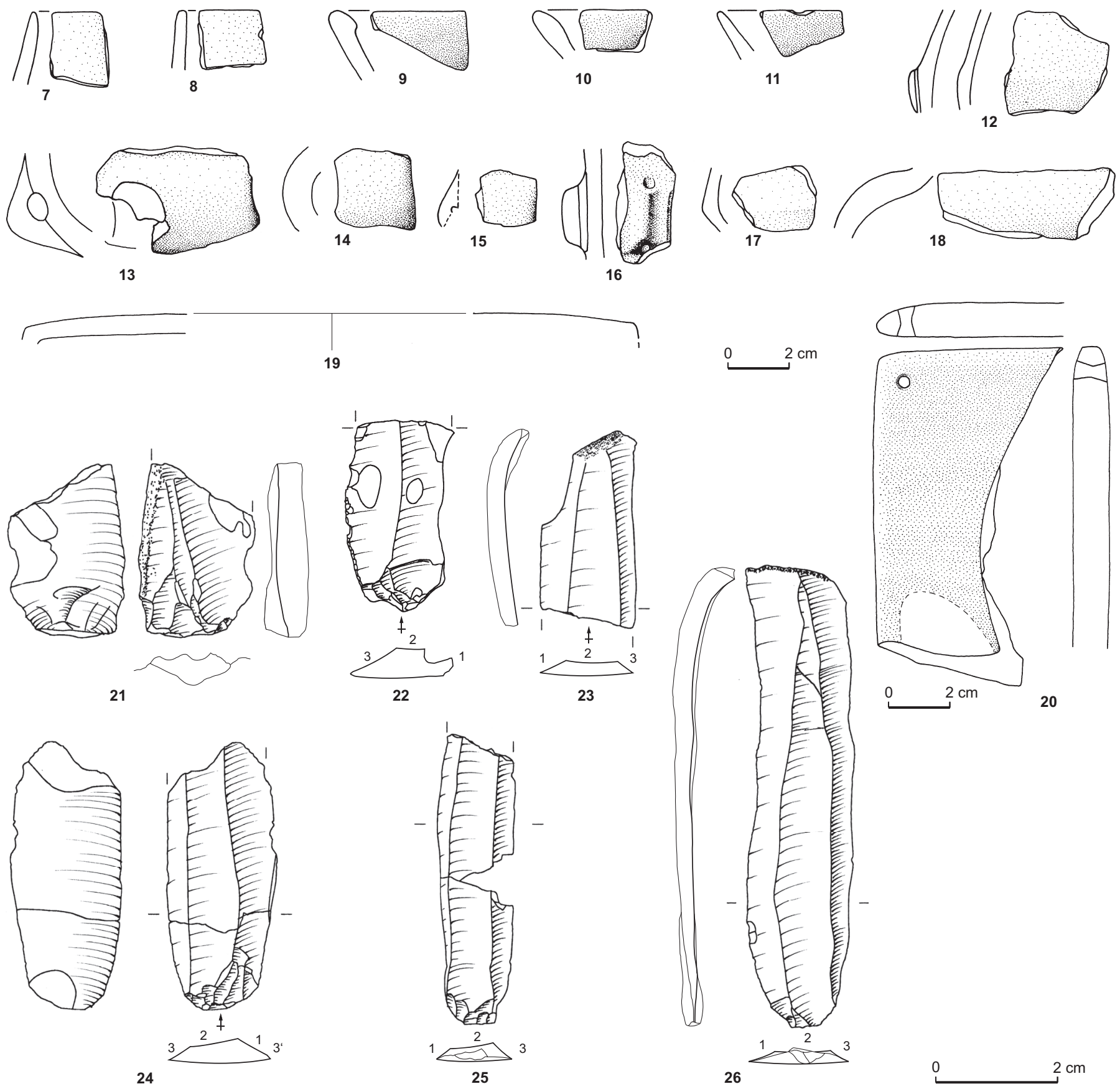

Fig. 26 - Mobilier céramique et industrie lithique de la structure FS 75 (dessin: S. Renault, CNRS et S. Van Willigen, Lampea). 
sur les deux fragments. La spectroscopie de fluorescence $\mathrm{X}$ a montré la présence exclusive de calcium dans l'incrustation sur le tesson rouge mais aussi un taux très haut de fer dans celle du tesson noir qui provient probablement de la céramique elle-même. Ceci peut s'expliquer par le fait que l'incrustation étant très mal conservée, le support, c'est-àdire la terre cuite, a été analysé simultanément. En ce qui concerne l'incrustation elle-même, aucun autre élément n'ayant pu être mis en évidence, il semblerait qu'il s'agisse d'un carbonate de calcium. Un échantillon (moins d'un milligramme) a été soumis à la spectrométrie infrarouge à transformée de Fourier. Les résultats confirment la présence de carbonate de calcium (pic caractéristique des carbonates situé à $1409,4 \mathrm{~cm}^{-1}$ ) et montrent clairement que l'incrustation ne contient aucune substance organique (fig. 25c).

Trois tessons font partie d'une plaque en terre cuite qu'il faut probablement interpréter comme une préhension de louche (fig. 24, no 4).

Le reste du mobilier céramique est constitué d'une série de vases très fragmentés parmi lesquels quelques éléments sont cependant dignes d'intérêt: un fragment de panse avec une préhension multitubulée (fig. 26, $\mathrm{n}^{\circ} 16$ ), une anse en ruban bien dégagée (fig. 26, no 3), un fragment correspondant à l'épaulement large d'un grand vase à col (fig. 26, $n^{\circ} 18$ ), deux tessons qui faisaient vraisemblablement partie d'un couvercle (fig. 26, no 19) et, pour finir, deux fragments qui correspondent à deux coupes à épaississement interne (fig. 26, $\mathrm{n}^{\text {os }} 9$ et 10).

L'industrie lithique comporte seize objets: un percuteur (?) sur galet en grès; cinq éclats dont deux en silex blond bédoulien, un en silex gris et deux brûlés; un éclat laminaire cortical; un fragment proximal d'éclat laminaire brûlé (fig. 26, no 21) ; un fragment proximal de lame brûlée (fig. 26, no 22); un fragment mésial de lamelle à section triangulaire en silex gris; un fragment mésial de lame brûlée à section triangulaire; un fragment distal de lame corticale (extrémité corticale du nucléus emportée) de section trapézoïdale, bords et nervures réguliers (code de débitage 1-2-3) (fig. 26, no 23); un fragment proximal de lame, brûlé, section trapézoïdale asymétrique, bord droit flottant et préparation du plan de frappe par réduction de la corniche et doucissage de l'arête (fig. 26, no 24); un fragment proximal de lame, brûlé, section trapézoïdale, bords et nervures relativement rectilignes et parallèles, préparation du plan de frappe par doucissage (code de débitage 1-2-3) (fig. 26, no 25); une lame (extrémité du nucléus emportée) de section trapézoïdale irrégulière, à nervures non rectilignes et bords présentant un aspect flottant. Le talon présente deux enlèvements (dièdre plat), le bulbe, un esquillement et une très légère lèvre, l'angle de chasse est droit et les ondulations sont marquées sur la face inférieure (code de débitage 1-2-3) (fig. 26, no 26).

Un fragment d'objet particulier attire l'attention (fig. 26, $\left.n^{\circ} 20\right)$ : il s'agit d'un angle de plaquette perforée déterminé par deux bords perpendiculaires régularisés, l'un est arrondi, l'autre est aplani. La longueur maximale du fragment est de 11,2 cm, la largeur de 6,1 cm. Dans l'angle, la perforation bitronconique a $0,4 \mathrm{~cm}$ de diamètre, elle est à $0,6 \mathrm{~cm}$ et $0,7 \mathrm{~cm}$ des bords. L'épaisseur maximale de l'objet est de $1,2 \mathrm{~cm}$, celle du bord arrondi est de $0,7 \mathrm{~cm}$, et celle du bord aplani est de $0,8 \mathrm{~cm}$. Le matériau est un calcaire gris induré en plaquette dont l'épaisseur initiale était probablement proche de celle de l'objet fini. On y distingue quelques grains de quartz. Ce type de roche n'appartient ni aux faciès urgoniens des monts de Vaucluse, ni aux assises du Miocène superposé de la plaine de Carpentras; elle pourrait provenir des couches de l'Oligocène de Fontaine-de-Vaucluse, mais cela reste peu vraisemblable. Il pourrait donc s'agir d'une roche sédimentaire d'origine extrarégionale ${ }^{9}$.

\section{QUELS SONT LES GROUPES CULTURELS REPRÉSENTÉS SUR LE SITE DES BAGNOLES ?}

Au-delà du constat de la présence de mobilier céramique original et inédit, les premières interrogations relatives à ces ensembles d'objets concernent leur attribution chronoculturelle et leur intégration aux schémas de l'évolution du Néolithique. Viennent ensuite les questions de leur signification et du fonctionnement du site, plus difficiles à traiter.

S'il est possible de rattacher ces ensembles au Néolithique moyen lato sensu, on remarque évidemment que cela reste imprécis et insatisfaisant. Il n'est cependant pas possible d'aller plus loin pour le mobilier qui provient des structures et des unités stratigraphiques FO 14, US 31, US 42 et US 67 : il est soit trop pauvre, soit peu spécifique. En revanche les structures et unités stratigraphiques SI 01, FS 13, FS 22, FS 47, FS 48, US 62, US 70 et FS 75 ont livré suffisamment d'éléments diagnostiques pour que l'on puisse en discuter d'une manière détaillée.

Une partie de ces ensembles présente des caractères céramiques traditionnellement considérés comme carac-

9. Détermination pétrographique par Jean-Pierre Masse, Université de Provence. 
téristiques du Chasséen. L'industrie lithique, malgré la reconnaissance (non exclusive) de silex bédoulien, ne livre pas d'élément spécifique de cette culture. On se heurte ici au problème de la terminologie employée et à celui du contenu attribué au Chasséen.

Les caractères de cette culture archéologique ont été fixés dans les années 1950 par les travaux de J. Arnal, G. Bailloud et R. Riquet à partir de la céramique du Camp de Chassey et de la grotte de la Madeleine: assiettes à marli, vases supports, écuelles à épaulement, vases cylindrosphéroïdaux, anses en flûte de Pan, coupes à sillon interne, cordons multiforés, décors gravés (Arnal, 1949, 1953 et 1956; Arnal, Bénazet, 1951; Bailloud, Mieg de Boofzheim, 1955; Riquet, 1959).

Au cours des décennies suivantes, un nombre considérable de sites, dont l'homogénéité est parfois discutable, est attribué au Chasséen. La liste des traits considérés comme caractéristiques de cette culture s'élargit continuellement à tel point que le Chasséen finit dans certaines régions par englober l'ensemble du Néolithique moyen. À partir des années 1980, la recherche se détourne de ces problèmes de définition pour aborder la question épineuse des évolutions régionales (Vaquer 1990b; Beeching, 2002). Sans entrer dans le détail, ces études conduisent à la distinction entre un pôle ancien caractérisé en particulier par les assiettes à marli, les assiettes à socle et les décors gravés (Chasséen ancien de J. Vaquer et groupe B de A. Beeching) et un pôle récent qui regroupe les coupes à sillon interne et les jattes et gobelets carénés ou à épaulement (Chasséen classique et récent de J. Vaquer et groupes C, D et E de A. Beeching).

Parallèlement, l'outillage lithique chasséen est défini par D. Binder sur la base du mobilier des couches 4 à 8 de la grotte de l'Église supérieure et des couches 10 à 24 de la Baume Fontbrégoua (Binder, 1984, 1991 et 1998; Binder, Gassin, 1988). Ce travail trouve un prolongement dans une série de monographies et d'articles publiés récemment (Briois, 2005; Léa, 2004a; Léa et al., 2007). Là encore, on retrouve la distinction entre un ensemble ancien caractérisé par un débitage sur nucléus (semi-) coniques sans traitement thermique et un ensemble récent marqué par l'apparition du débitage lamellaire à la pression après traitement thermique sur nucléus (semi-)coniques puis quadrangulaire plat.

Aujourd'hui, la plupart des auteurs s'accordent pour considérer le Chasséen comme un complexe culturel qui présente une grande variabilité, tant sur le plan de la culture matérielle que sur celui des pratiques funéraires. Les productions sont caractérisées par les éléments suivants : assiettes à marli, assiettes à socle, anses en flûte de Pan, vases à épaulement, micro-épaulements, jattes et gobelets carénés, coupes à sillon(s) interne(s), cordons multiforés et décors gravés, utilisation de silex blond bédoulien, débitage à la pression sur silex chauffé, débitage sans traitement thermique, nucléus (semi-)coniques, nucléus quadrangulaires plats. Même si l'on s'accorde actuellement pour dire que certains de ces éléments sont plutôt caractéristiques d'un pôle ancien (Proto-Chasséen et Chasséen ancien) alors que d'autres sont limités à un pôle récent (Chasséen classique et Chasséen récent), tous sont considérés comme caractéristiques du Chasséen. Cette définition est extrêmement large et ne tient pas compte des profondes différences qui existent entre le pôle ancien et le pôle récent. Si les assiettes à marli, les assiettes à socle et les anses en flûte de Pan sont spécifiques du Chasséen, que faire des ensembles qui n'ont livré aucun de ces éléments ? À l'inverse, si ce sont les jattes à épaulement et les coupes à sillon(s) interne(s) qui sont spécifiques du Chasséen, que faire des ensembles qui n'ont livré que des assiettes à socle associées à un outillage lithique issu d'un débitage semi-conique ? Ce problème est posé en quelques mots par S. Cassen et P. François dans un article consacré au Néolithique moyen de l'ouest de la France (Cassen, François, 2006): «Par ailleurs, admettre que l'Auzay-Sandun n'est rien de moins, ou de plus, que du Chasséen supposerait que nous soyons en mesure de savoir précisément ce qui fait le Chasséen. Or depuis Arnal, personne n'a été capable d'expliquer ce qui était à la fois nécessaire et suffisant pour avoir "du" Chasséen, qu'il soit méridional ou du Bassin parisien. »

Avant de pouvoir se prononcer sur une attribution éventuelle de l'ensemble ou d'une partie du mobilier des Bagnoles au Chasséen, il est donc nécessaire de faire le point sur le sens qu'il est possible d'accorder actuellement à ce terme.

\section{LE CHASSÉEN : UNE PROPOSITION DE DÉFINITION}

Ces dernières années, le dynamisme de la recherche a été matérialisé par la publication de plusieurs ouvrages consacrés à des sites de référence tels que le Camp de Chassey et Villeneuve-Tolosane (Thevenot, 2005; François, 2007). Dans le même temps, plusieurs ensembles clos ou stratifiés ont été découverts: structures en creux des sites de Jardins de Vert-Parc et de Jacques-Cœur II (Georjon, 2003), stratigraphies de Villa Giribaldi (Binder, 1990 et 2004; Binder et al., 2008) et de la grotte du Mourre de la Barque (fouilles Van 
Willigen). Ces travaux et découvertes récentes ont conduit à un renouvellement de nos connaissances et permettent d'aborder le problème de la définition du Chasséen.

Nous proposons donc de fonder ce travail sur les ensembles clos ou sur les ensembles stratifiés. Dans le premier cas, qu'il s'agisse de fosses dépotoirs, dépôts ou encore de mobilier funéraire, on peut supposer que les éléments qui le constituent correspondent à une période relativement brève de l'ordre de quelque dizaines d'années. Les ensembles stratifiés peuvent, quant à eux, correspondre à une plus grande durée, résultat du temps de formation d'un niveau archéologique et des inévitables problèmes taphonomiques, ce qui en fait une source de moindre qualité. Cependant, ce type d'ensemble étant encore relativement fréquent dans la zone d'étude, nous nous sommes résolus à les utiliser, à condition naturellement qu'ils aient fait l'objet d'une fouille stratigraphique. La réduction de notre base de travail aux ensembles clos ou stratifiés présente certes l'inconvénient de limiter considérablement la documentation. Généralement, ces ensembles ne livrent que quelques artefacts accompagnés d'un peu de faune. Il est donc très tentant de travailler à l'échelle du site. Ceci n'est envisageable qu'au prix d'une perte de fiabilité, inacceptable à une époque où la recherche doit mettre l'accent sur une plus grande précision dans ses classifications chronologiques et culturelles. Par ailleurs, le nombre restreint de documents provenant des ensembles clos ou stratifiés est compensé par la quantité croissante de ces derniers, notamment suite aux travaux d'archéologie préventive. L'association de deux éléments au sein d'un ensemble clos peut être le fruit du hasard. Par contre, cette même association dans plusieurs ensembles clos a une signification précise. Il en va de même pour les exclusions: il y a une forte probabilité pour que deux éléments jamais associés entre eux soient diachrones et/ou appartiennent à des cultures archéologiques différentes.

Une série de types céramiques figure à la fois dans la définition princeps du Chasséen proposée par J. Arnal et R. Riquet et au sein du mobilier attesté sur le site éponyme: assiettes à marli, assiettes à socle, décors gravés/incisés sur le marli ou sur le socle, anses en flûte de Pan. Or, il existe dans la moitié sud de la France une série d'ensembles clos ou stratifiés qui ont livré plusieurs de ces éléments: Villa Giribaldi, niveaux chasséens à Nice dans les Alpes-Maritimes (Binder, 1990 et 2004); Saint-Michel-du-Touch, structure A 185 à Toulouse en Haute-Garonne (Méroc, Simonnet, 1979; CapJédikian et al., 2008) ; les Monges, structure F1 à Launaguet en Haute-Garonne (Servelle, 1980); Camp de Chassey, niveaux 8 et 9 à Chassey en Haute-Saône (Thevenot, 2005); le Pirou, couche 3 à Polignac en Haute-Loire (Houdré, Vital, 1979); Jacques-Cœur II, structure 22 à Montpellier dans l'Hérault (Jallot et al., 2000; Georjon, 2003); Jardins de Vert-Parc, structure 1095 à Castelnau-le-Lez dans l'Hérault (Georjon, 2003; Briois, Léa, 2003); grotte du Mourre de la Barque, niveau Q à Jouques dans les Bouches-du-Rhône (fouilles Van Willigen). Nous proposons d'utiliser ces neuf ensembles clos ou stratifiés comme référence pour proposer une définition du Chasséen.

Ces ensembles partagent une série de caractères céramiques (tabl. II). Les formes les plus fréquentes sont les vases à inflexion basse et col rectiligne fermé, les vases à inflexion basse et col concave fermé ou vertical, les vases à profil galbé, les assiettes à socle, les coupes en calotte à lèvre droite, les assiettes à marli et aile rectiligne large, les coupes en calotte à épaississement interne rectiligne et décrochement vif, les jattes à inflexion basse et col rectiligne ouvert, les assiettes à marli et aile horizontale convexe et les coupes en calotte à bourrelet interne. Parmi les préhensions, il faut mentionner en particulier les anses en ruban sous le bord et les anses tunneliformes horizontales sur l'inflexion. Les décors sont constitués d'incisions disposées en bandes, triangles ou losanges quadrillés qui ménagent parfois des bandes réservées. Ils sont limités aux assiettes à marli et aux assiettes à socle. Une série de caractères pourraient, bien qu'ils soient plus rares, faire partie de ce même type d'assemblage céramique: les grands vases à bord épaissi à l'extérieur, les languettes multiforées, les préhensions multitubulées, les épaulements partiels et les vases à col haut.

En ce qui concerne l'industrie lithique de ces neuf ensembles, la quantité de pièces varie fortement; en dehors de Giribaldi et Chassey, ces séries lithiques sont numériquement faibles et peu diagnostiques. Lorsque les matières premières sont déterminées, on constate que le silex blond bédoulien (du Vaucluse ?) est faiblement majoritaire ou légèrement minoritaire; il fournit une bonne partie ou la plus grande partie des lames et/ou des outils. Dans les ensembles numériquement significatifs, les lamelles et petites lames sont principalement débitées par percussion indirecte sur nucléus conique/semi-conique. Les nucléus quadrangulaires plats ne sont pas formellement attestés. Les témoignages de l'utilisation de la pression et surtout de la chauffe sont rares ${ }^{10}$. Les armatures sont principalement géométriques (armatures tranchantes), et il y a peu

10. Une lame débitée par pression sans traitement thermique a été trouvée dans la structure A185 de Saint-Michel-du-Touch et trois parmi le mobilier de la structure 22 de Jacques-Cœur II. 
Tabl. II - Récapitulatif des éléments représentés dans les assemblages de référence mentionnés page 211 et dans les structures 13, 47, 48 et 75 des Bagnoles (DAO: S. Van Willigen, Lampea).

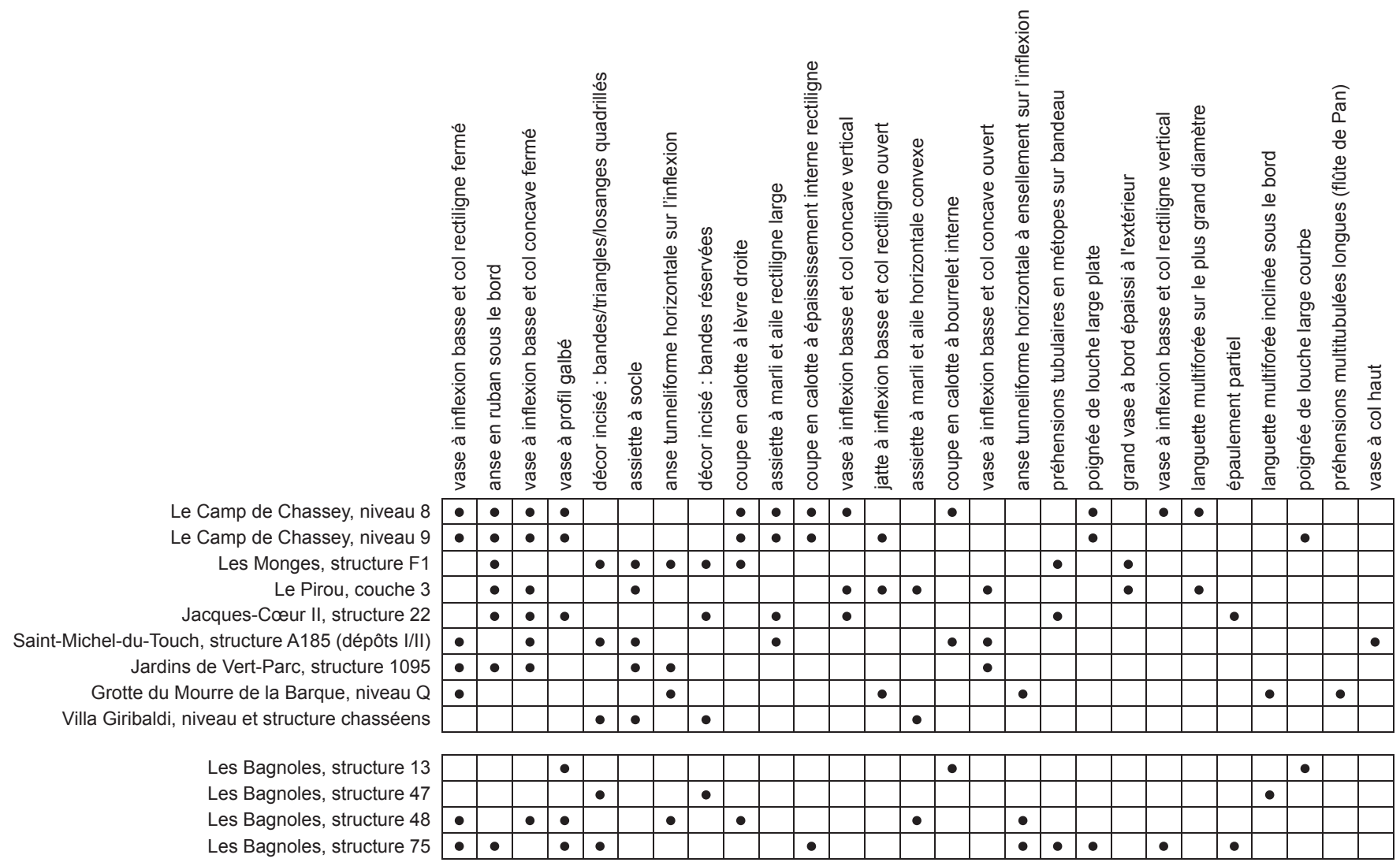

de pièces foliacées bifaces. Dans l'outillage, les burins et les chanfreins sont peu nombreux. Dans ces conditions, et comme l'ont déjà démontré un certain nombre de chercheurs, il est difficile de considérer le débitage par pression sur nucléus chauffés coniques ou quadrangulaires plats comme une caractéristique de ces ensembles (Briois, Léa, 2003; Léa et al., 2004; Léa, 2004b; Léa et al., 2007; Briois et al., 2008).

Le Chasséen défini de la sorte correspond donc au pôle ancien dont il a été question ci-dessus, c'est-à-dire au Chasséen ancien de J. Vaquer et au groupe B de A. Beeching (Vaquer, 1990b; Beeching, 2002). Les datations radiocarbone obtenues dans ces ensembles clos ou stratifiés de référence ${ }^{11}$ permettent de le placer dans la seconde moitié du $V^{e}$ millénaire avant notre ère (fig. 27 et tabl. III).

11. Seules les datations qui proviennent des ensembles de référence mentionnés page 211 et qui ont un écart standard inférieur à cent ans ont été utilisées ici.
Si l'on s'en tient à cette définition, il est possible de parler de Chasséen sans spécification géographique. En effet, cette culture archéologique n'est pas limitée au Midi méditerranéen puisque des sites continentaux tels que le Camp de Chassey, le Pirou et Amboise ${ }^{12}$ (Irribaria, 2006) correspondent tout à fait à la définition proposée ci-dessus. Dans le même ordre d'idée, les termes de Chasséen garonnais I dans la vallée de la Garonne (François, 2007), de Chasséen de type les Plots dans la plaine de l'Aude (Vaquer, 1990b), de Chasséen ancien dans la basse vallée du Lez (Georjon, 2003), de groupe B de la moyenne vallée du Rhône (Beeching, 2002), s'ils sont utiles, contribuent cependant à masquer l'unité du phénomène chasséen. À l'inverse, le terme «Chasséen ancien» suggère une continuité culturelle avec les groupes de la première moitié du

12. Nous nous contentons ici d'évoquer ce site sans en tenir compte dans notre définition du Chasséen puisqu'il ne s'agit pas d'un ensemble clos. 


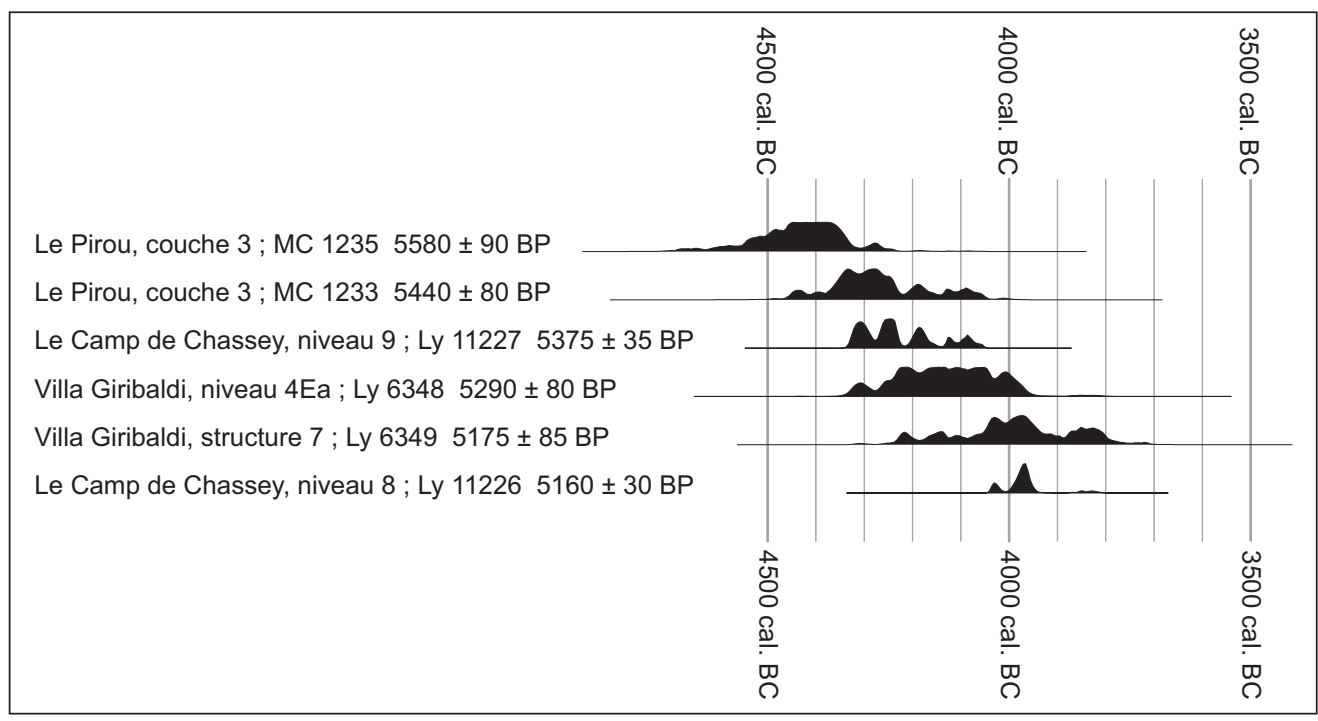

Fig. 27 - Histogrammes des probabilités des datations radiocarbone calibrées provenant des assemblages de référence mentionnés page 211. Les datations ayant un écart standard inférieur ou égal à cent ans ont été calibrées à l'aide du logiciel OxCal (version 3.10) et de la courbe de calibration de 2004 (Reimer et al., 2004) (DAO: S. Van Willigen, Lampea).

Tabl. III - Liste des datations radiocarbone provenant des assemblages de référence mentionnés page 211 (DAO: S. Van Willigen, Lampea).

\begin{tabular}{|l|c|c|c|c|l|}
\hline \multicolumn{1}{|c|}{ Site } & $\begin{array}{c}\mathbf{N}^{\circ} \\
\text { Labo }\end{array}$ & Date BP & $\begin{array}{c}\text { Niveaul } \\
\text { structure }\end{array}$ & Échantillon & $\begin{array}{c}\text { Référence } \\
\text { bibliographique }\end{array}$ \\
\hline Le Pirou & MC 1235 & $5580 \pm 90$ & couche 3 & $?$ & Houdré et Vital, 1979 \\
\hline Le Pirou & MC 1233 & $5440 \pm 80$ & couche 3 & charbon & Houdré et Vital, 1979 \\
\hline Le Camp de Chassey & Ly 11227 & $5375 \pm 35$ & niveau 09 & charbon & Thevenot, 2005 \\
\hline Villa Giribaldi & Ly 6348 & $5290 \pm 80$ & niveau 4Ea & $?$ & Gassin et al., 2003 \\
\hline Villa Giribaldi & Ly 6349 & $5175 \pm 85$ & $\begin{array}{c}\text { structure 7/ } \\
\text { niveau 4h/5a }\end{array}$ & $?$ & Gassin et al., 2003 \\
\hline Le Camp de Chassey & Ly 11226 & $5160 \pm 30$ & niveau 08 & charbon & Thevenot, 2005 \\
\hline
\end{tabular}

IV millénaire alors que, tout au moins dans le quart sud-est de la France, la période postérieure à 4000/3900 av. J.-C. est marquée par l'abandon de la majorité des formes et décors céramiques que nous considérons comme spécifiques du Chasséen, et par l'apparition de nouveaux types céramiques et de nouvelles techniques de débitage (voir par exemple des ensembles datés du début du IV $V^{\mathrm{e}}$ millénaire avant notre ère tels que les structures 39 et 1020 des Jardins de Vert-Parc ou encore les structures 46 et 131 de la Roberte: Georjon, 2003; Beeching, 2002).

\section{LES BAGNOLES: LES STRUCTURES ATTRIBUABLES AU CHASSÉEN}

\section{STRUCTURE FS 13}

Parmi les quelques éléments diagnostiques qui proviennent de cette structure, trois vases font clairement référence au Chasséen. Le vase à profil légèrement galbé et lèvre amincie (fig. $4, \mathrm{n}^{\circ} 1$ ) est une forme que l'on retrouve au sein du mobilier de la structure 22 du site de Jacques-Cour II (Georjon, 2003, fig. 5, n ${ }^{\text {os }} 5$ et 7), mais aussi dans les structures 22 (fig. 7, $\mathrm{n}^{\circ}$ 1) et 75 (fig. 23, $\mathrm{n}^{\circ}$ 4) des Bagnoles. La petite coupe en calotte à épaississement interne (fig. 4, $n^{\circ} 5$ ) est une forme attestée dans les autres assemblages de référence du Chasséen: le niveau 8 du Camp de Chassey (Thevenot, 2005, fig. 73, $\mathrm{n}^{\circ}$ 14) et la structure A 185 de Saint-Michel-du-Touch (Méroc, Simonnet, 1979, fig. 15, K). Le dernier élément diagnostique de cette structure est la poignée large et courbe qui peut avoir servi de préhension à une coupe (fig. $4, \mathrm{n}^{\circ} 6$ ). De telles louches à poignée large selon la terminologie de J. Vaquer (Vaquer, 1975) sont attestées au sein du mobilier du niveau 9 du Camp de Chassey (Thevenot, 2005) (fig. $40, \mathrm{n}^{\text {os }} 9$ et 11).

L'industrie lithique de cette structure, en dehors de l'usage de silex bédoulien, ne montre aucun caractère 
particulier. On remarque ainsi qu'il n'y a pas d'indice de débitage par pression après chauffe.

\section{STRUCTURE FS 22}

La pauvreté de cet assemblage ne permet pas d'en proposer une attribution culturelle précise. Tout au plus est-il possible de constater la ressemblance du vase à profil galbé (fig. $7, \mathrm{n}^{\circ} 1$ ) avec les exemplaires du même type qui proviennent des structures FS 13 et FS 75 et de proposer, à titre d'hypothèse, d'attribuer le remplissage de la structure FS 22 au Chasséen.

L'industrie lithique est ici encore peu abondante et ne contredit pas cette proposition. Le silex bédoulien est majoritaire mais on doit également remarquer l'absence de débitage de lamelle par pression après traitement thermique des nucléus.

\section{STRUCTURE FS 47}

Un élément provenant de cette structure permet de l'attribuer au Chasséen tel qu'il a été défini ci-dessus: le décor d'incisions disposées en triangles quadrillés des deux vases à corps cubique (fig. 11 et 12) est relativement fréquent sur les assiettes à marli et sur les assiettes à socle comme les exemplaires du niveau 9 du Camp de Chassey (Thevenot, 2005, fig. 48, $\mathrm{n}^{\text {os }} 4-5$ ) ou encore celui de la structure 22 du site de Jacques-Cœur II (Georjon, 2003, fig. $6, \mathrm{n}^{\mathrm{o}} 1$ ).

Le vase sphérique muni d'une barrette multiforée (fig. 10, $\mathrm{n}^{\mathrm{o}} 3$ ) est comparable aux exemplaires du Camp de Chassey (Thevenot, 2005, fig. 72, no 2) et du niveau MB03-3 de la grotte du Mourre de la Barque (fouilles Van Willigen). Cependant, on le retrouve également dans des ensembles plus récents tels que la couche 6 de la grotte de l'Église à Baudinard dans le Var (Courtin, 1967, fig. 13) mais aussi en contexte Montbolo: couche 11 de la grotte de Montou, galerie Fabresse, couloir 1 à Corbère-les-Cabanes dans les Pyrénées-Orientales (Claustre, Ponsich, 2000-2001, fig. 11, $n^{\circ} 3$ ) et galerie close de la même grotte (Ponsich, TreinenClaustre, 1990, fig. 12, no 4). Il ne semblerait donc pas qu'il s'agisse là d'un trait véritablement spécifique du Chasséen. Les deux vases à dégraissant grossier (fig. 10, $\mathrm{n}^{\text {os }} 1-2$ ) se démarquent du reste du mobilier céramique par leur dégraissant, leur couleur et leur forme. Ces deux récipients sont peu spécifiques. Les meilleurs éléments de comparaison se trouvent au sein des assemblages attribués au groupe de Saint-Uze : la Vieille Église à la Balme-de-Thuy en Haute-
Savoie (Beeching et al., 1997, fig. 7, $\mathrm{n}^{\circ}$ 9) et la grotte du Gardon à Ambérieu-en-Bugey dans l'Ain (Beeching et al., 1997, fig. $6, \mathrm{n}^{\mathrm{o}} 1$ ).

L'industrie lithique de la structure ne livre aucune information utile. Le mobilier de la structure FS 47 peut donc être, au moins en partie, attribué au Chasséen. Cependant, quelques éléments peuvent éventuellement faire référence au Saint-Uze, groupe culturel contemporain du Chasséen et centré sur la moyenne vallée du Rhône.

\section{STRUCTURE FS 48}

L'assiette à marli et aile étroite et convexe et à décor externe (fig. 15 et 16) n'est actuellement attestée que dans les niveaux chasséens de Villa Giribaldi à Nice dans les Alpes-Maritimes (Binder, 1990, fig. $1, \mathrm{n}^{\text {os }} 1$ et 3 ). Nous reviendrons sur ce type de vase qui est intéressant dans la mesure où, comme l'a déjà souligné $\mathrm{D}$. Binder à propos de l'exemplaire de Giribaldi, il présente des affinités avec certaines formes céramiques de la phase méandro-spiralique des Vasi a Bocca Quadrata (VBQ).

Nous ne nous arrêtons ici sur les vases peints que pour signaler qu'ils présentent, tous les trois, des formes bien attestées dans le Chasséen et le Montbolo (fig. 17, n ${ }^{\text {os }} 1-3$ ). Les meilleurs points de comparaison pour le vase à carène basse et col concave fermé (fig. 17, $\mathrm{n}^{\mathrm{o}} 1$ ) proviennent de contextes attribuables au groupe de Montbolo et au Chasséen. Dans la sépulture de la Bassa à Fonteta dans le district de Gérone (Tarrús et al., 1982), un vase à carène basse et col concave comparable au vase qui nous intéresse ici, si l'on fait abstraction des deux anses tubulaires verticales caractéristiques du groupe de Montbolo, était associé aux fragments d'une assiette à socle ornée de motifs gravés. Un autre exemplaire comparable, bien que sensiblement moins fermé, provient de la structure 1068 des Jardins de Vert-Parc à Castelnau-le-Lez dans l'Hérault (Georjon, 2003, fig. $10, n^{\circ} 1$ ). Le deuxième vase peint (fig. 17, $n^{\circ} 2$ ) se distingue du premier seulement par son col rectiligne qui permet de le rapprocher des vases de la structure 1068 du site des Jardins de Vert-Parc (Georjon, 2003, fig. 11, no 2), de la fosse du Lavous à Grand-Gallargues dans le Gard (Fenouillet, Vaquer, 1974, fig. 2, $\mathrm{n}^{\mathrm{o}} 2$ ), du niveau $Q$ de la grotte du Mourre de la Barque (fouilles Van Willigen) et d'un vase de la sépulture A185 (dépôt 2) de Saint-Michel-du-Touch (Méroc, Simonnet, 1979, fig. 15, D). Il rappelle aussi, par ses proportions, un vase malheureusement mal conservé et partiellement reconstituable de la structure FC 2 de la Terrasse à Villeneuve-Tolosanne en Haute-Garonne 
(Vaquer, 1990a, fig. 115, no 3). Par sa forme et ses proportions, le vase peint à profil galbé (fig. $17, n^{\circ} 3$ ) est, quant à lui, comparable à certaines formes de la galerie close de la grotte de Montou à Corbère-les-Cabanes dans les PyrénéesOrientales (Ponsich, Treinen-Claustre, 1990, fig. 8, $n^{\text {os }} 1,3$ et 4), ensemble attribué au groupe de Montbolo, ainsi qu'à un des vases de la structure 22 de Jacques-Cœur II attribuée au Chasséen (Georjon, 2003, fig. 6, no 2).

Un élément, l'assiette à marli et aile étroite convexe, permet d'attribuer le remplissage de cette structure au Chasséen. Les trois vases peints ont, quant à eux, des formes bien attestées dans les ensembles de référence chasséens mais ils sont également présents en contexte Montbolo. Leur décor, sur lequel nous reviendrons ci-dessous, est par contre actuellement sans équivalent dans le contexte du Néolithique moyen régional. Pour mémoire, il n’y a pas d'industrie lithique dans cette structure.

\section{STRUCTURE FS 75}

Cette fosse a livré un matériel céramique relativement abondant dont quatre formes et l'ensemble des décors sont spécifiques du Chasséen. La coupe en calotte à épaississement interne rectiligne et décrochement vif est attestée dans quelques assemblages chasséens (fig. 23, $n^{\circ} 1$ ): les structures 1073 et 1077 d'Encombres à Quarante dans l'Hérault (Amiel, Jédikian, 2003, fig. 1, 8 et 10), le Pereiras, point 15 à Pouzols dans l'Aude (Ambert et al., 1988, fig. 10), Cugnaux, structures CX 17, CX 18 et CX 22 à VilleneuveTolosane dans la Haute-Garonne (François, 2002) et le Pirou à Poulignac en Haute-Loire (Houdré, Vital, 1979, fig. 9, $\mathrm{n}^{\circ} 2$ ). Il faut cependant signaler que ce type de coupe est également attesté en contexte Montbolo à la grotte de la Chance, niveaux N4f6-7 à Ria dans les PyrénéesOrientales (Baills, 1987) et dans la galerie Fabresse de la grotte de Montou à Corbère-les-Cabanes dans les PyrénéesOrientales (Claustre, Ponsich, 2000-2001, fig. 8, $\mathrm{n}^{\circ}$ 3). De telles coupes sont aussi connues en Italie en contexte VBQ et chasséens: Palù di Livenza à Pordenone, FriuliVenezia Giulia (Peretto, Taffarelli, 1973, fig. 1, $\mathrm{n}^{\text {os }} 5$ et 7), Colombare à Negrar dans le Veneto (Fasani, Visentini, 2002, fig. 3, $\mathrm{n}^{\mathrm{o}}$ 2), Isolino à Biandronno en Lombardie (Guerreschi, 1976-1977, tav. XIV, no 1566 et LXV, no 5696) et à Quadrato di Torre Spaccata à Rome (Anzidei, Carboni, 1995, fig. $40, \mathrm{n}^{\text {os }} 4$ et 5 ).

Le vase à épaulement partiel (fig. 23, $\mathrm{n}^{\circ} 4$ ) ressemble, par sa forme galbée et la lèvre amincie, aux vases des structures 13 et 22 des Bagnoles et de la structure 22 du site de
Jacques-Cœur II (Georjon, 2003, fig. 5, no 4 et fig. 6, no 2). La présence d'un épaulement limité à l'espace existant entre deux préhensions se retrouve sur un grand fragment de panse qui provient de la même structure 22 de JacquesCœur II (Georjon, 2003, fig. 5, no 3), à cette différence près que la seule préhension conservée est dans ce cas un bouton perforé verticalement.

Le vase à bandeau multiforé et cannelé (fig. 24, $\mathrm{n}^{\circ} 1$ ) est attesté dans quelques assemblages chasséens tels que l'abri de Font-Juvénal, couche 10inf/sup à Conques-sur-Orbiel dans l'Aude (Guilaine et al., 1990, fig. 4, no 10) et la fosse 1 des Monges à Launaguet en Haute-Garonne (Servelle, 1980, fig. 6). Malgré certaines différences, il est comparable au vase à bandeau multiforé de la structure 22 de JacquesCour II (fig. 30, n⿳0 6).

Les assiettes à socle décorées sont attestées dans la structure 1095 des Jardins de Vert-Parc (Georjon, 2003, fig. 13) où un petit fragment décoré de triangles gravés quadrillés était associé à des vases à carène basse et col fermé comparables à ceux des structures FS 48 et FS 75 des Bagnoles (fig. 23, no 2). La partie inférieure d'une assiette à socle ajouré décoré de triangles, losanges et chevrons quadrillés provient des niveaux chasséens du site de Villa Giribaldi à Nice dans les Alpes-Maritimes (Binder, 1990, fig. 1, $\mathrm{n}^{\mathrm{o}} 4$ ). Le motif de bandes quadrillées organisées en chevrons n'est pas sans rappeler le décor d'une des assiettes à socle du niveau 8 du Camp de Chassey (fig. 30, $n^{\circ} 5$ ), et éventuellement celui visible sur un vase support de la structure VT-FC 2 de Villeneuve-Tolosane en Haute-Garonne (Vaquer 1990a, fig. 116).

Dans la structure FS 75, l'industrie lithique peu abondante témoigne de l'utilisation de silex bédoulien pour la production de lames débitées par pression sans traitement thermique des nucléus. Le fragment de plaquette perforée en calcaire (fig. 25, no 20) fait référence à deux tablettes comparables mais en grès qui proviennent de la structure A 185 de Saint-Michel-du-Touch (Méroc, Simonnet, 1979, fig. 14, P1 et P2; Beyneix, 1997; Cap-Jédikian et al., 2008). Elles étaient déposées à proximité d'une concentration d'ossements humains et d'une assiette à marli et aile large décorée. La présence de tablettes perforées a été par ailleurs évoquée dans le cas de la structure 36 des Plots à Berriac dans l'Aude (Jallot et al., 2000, fig. 8) et de la sépulture en coffre de l'Arca de Calahons à Catllar dans les Pyrénées-Orientales (Jallot et al., 2000, fig. 8). Cependant, dans ces deux derniers cas, aucune mention n'est faite de la présence de telles tablettes dans les publications d'origine (Duday, Vaquer 2003; Abélanet, 1970). 


\section{LES STRUCTURES NON ATTRIBUABLES AU CHASSÉEN}

Trois structures et unités stratigraphiques n'ont livré aucun élément rentrant dans la définition du Chasséen proposée ci-dessus.

\section{STRUCTURE SI 01}

Cette structure, relativement pauvre, n'a livré que deux éléments diagnostiques. La grande jatte à carène basse et col concave vertical est relativement ubiquiste dans le Néolithique moyen du sud-est de la France et ne permet pas de préciser la datation de cet assemblage (fig. 3, no 2). En revanche, la jatte carénée à col concave vertical et partie inférieure concave (fig. $3, \mathrm{n}^{\circ} 1$ ) est une forme que l'on retrouve dans des assemblages datés de la première moitié du IV millénaire cal. BC: couche 5 de la grotte $\mathrm{C}$ de Baudinard dans le Var (Courtin, Pélouard, 1971, fig. 13), de la couche la de la grotte IV à Saint-Pierre-de-la-Fage dans l'Hérault (G.-B. Arnal, 1988, fig. 2, no 2 et fig. 3, no 3 ) et structure 39 du site de Plaine de Chrétien à Montpellier dans l'Hérault (Georjon, 2003). Cet élément permet donc d'envisager une attribution du silo SI 01 à la première moitié du $\mathrm{IV}^{\mathrm{e}}$ millénaire avant notre ère. L'industrie lithique ne livre aucune information utile.

\section{UNITÉ STRATIGRAPHIQUE US 62}

Le petit vase à préhension sur épaulement (fig. 20, $n^{\circ} 3$ ) trouve des points de comparaison au sein du mobilier des sites de la Plaine, silo 2 à Trets dans les Bouches-duRhône (Courtin, 1974, fig. 62, n ${ }^{\text {os }} 4$ et 6), du Chemin d'Aix, structure 2118 (fouilles L. Martin) à Saint-Maximin-laSainte-Baume dans le Var, de la grotte du Mourre de la Barque, niveau 18.3 (fouilles S. Van Willigen) à Jouques dans les Bouches-du-Rhône et des Martins, structure 6a (fouilles A. D'Anna) à Roussillon dans le Vaucluse. Ces ensembles peuvent être attribués à la première moitié du IVe millénaire. L'industrie lithique témoigne de l'utilisation de silex bédoulien pour la production d'éclats et de lames débitées par pression sans traitement thermique des nucléus.

\section{STRUCTURE US 70}

Le vase tronconique, seul élément diagnostique qui provient de cet ensemble, correspond à un type relativement rare dans le Néolithique moyen régional (fig. 22, no 1). Sa présence sur le site de la ZAC des Laurons à Nyons dans la Drôme, phases D-E (Beeching et al., 2004, fig. 7), au sein du mobilier de la couche 3 de la grotte de l'Église à Baudinard dans le Var (Courtin, 1967, fig. 20) et de la Lagozza à Besnate en Lombardie (Guerreschi, 1967, fig. 147 et 153) semble indiquer qu'il s'agit là d'un type de vase attribuable à la première moitié du IV millénaire. L'industrie lithique ne livre aucune information utile.

\section{STRUCTURE, ATTRIBUTION CULTURELLE ET FONCTION DU SITE}

L'opération réalisée sur le site des Bagnoles à l'Isle-surla-Sorgue n'ayant porté que sur environ $5 \%$ de l'emprise, il est évident que toute interprétation concernant la structure et la fonction du site est extrêmement fragile. Ainsi, il est, en l'état actuel des données, impossible de dire si la zone diagnostiquée se trouve au centre ou en périphérie de l'établissement néolithique. De même, l'étendue de ce dernier ne peut être fixée. On peut, par ailleurs, se demander si nous avons affaire à un seul site ou à deux sites distincts, l'un matérialisé par les structures du secteur sud-ouest, l'autre au nord-est de la «roubine» et identifié par le seul silo SI 01. Malgré ces incertitudes liées à la nature de l'opération, il est possible de faire un certain nombre de constatations.

Le diagnostic a conduit à la fouille de douze structures attribuées au Néolithique moyen d'après les caractéristiques de la céramique et/ou de l'outillage lithique qu'elles ont livré (tabl. I). Cinq de ces structures (FS 13, FS 22, FS 47, FS 48 et FS 75) peuvent être attribuées au Chasséen tel que nous avons proposé de le définir ci-dessus. Les structures SI 01, US 62 et US 70 semblent être plus tardives. Les quatre structures restantes (FO 14, US 31, US 42, US 67) peuvent être attribuées au Néolithique moyen sans plus de précision.

La majorité des structures en creux du Néolithique moyen (SI 01, FS 13, FO 14, FS 22 et FS 70) renfermait du mobilier (céramique et lithique) fragmenté qui ne semble pas être le résultat d'une sélection délibérée mais plutôt d'un comblement lié à une activité domestique voisine. En revanche, deux fosses (FS 47 et FS 48) ont livré un assemblage particulier constitué chacun de vases en grande partie reconstituables, dont certains ne trouvent aucune comparaison sur le plan régional, accompagnés de quelques restes humains carbonisés et fragmentés. Malheureusement, il n'est plus possible de dire si ces vases ont été déposés intacts ou fragmentés dans la fosse et où ils 
se trouvaient par rapport aux restes humains. Seul un vase (vase à corps cubique ou socle) de la structure 47 semble avoir fait l'objet d'un traitement particulier (bris et crémation; voir supra, page 203).

Ces deux assemblages particuliers (FS 47 et FS 48) pourraient être le résultat d'un rituel funéraire encore difficile à cerner mais dans lequel incinération et dépôt (destruction ?) de céramique jouent un rôle particulier. Ce schéma (crémation humaine et dépôt de vases) viendrait alors compléter d'autres combinaisons documentées ces dernières années dans le Chasséen: dépôt partiel de vases brisés à proximité d'une inhumation sur le site JacquesCœur II, structure 22 (Jallot et al., 2000), dépôt partiel de vases brisés associé à une inhumation aux Plots, sépulture 37, à Berriac dans l'Aude, dépôt de vases détruits par bris et crémation associés à une inhumation aux Plots, sépulture 36 et fosse 35, à Berriac (Duday, Vaquer, 2003).

Le cas de la fosse FS 75 est plus difficile à interpréter. La céramique y est en partie très fragmentée et les fragments jointifs sont rares; l'outillage lithique ne semble pas avoir fait l'objet d'une sélection. Il faut cependant signaler la présence dans cette même structure d'un vase complet et d'un fragment de tablette perforée attestée par ailleurs en contexte funéraire (voir supra, page 225).

Les données recueillies montrent que le site des Bagnoles semble particulièrement vaste. Du point de vue chronologique, les structures du Néolithique moyen correspondent à au moins deux phases d'occupation. La première, localisée dans le secteur sud-ouest du diagnostic, est attribuable au Chasséen; elle s'étend sur un peu plus de six hectares, ce qui est un minimum compte tenu de la limite de l'emprise de l'évaluation. Elle paraît être en partie liée à une fonction particulière du site en rapport avec un rituel funéraire. Les trois structures les plus riches du site, à savoir FS 47, FS 48 et FS 75, correspondent à cette phase d'occupation et peuvent être considérées comme des ensembles de référence pour le Chasséen au même titre que les niveaux 7-9 du Camp de Chassey, la structure 22 de Jacques-Cœur II ou la structure A 185 de Saint-Michel-du-Touch. La seconde phase est attestée par les structures SI 01, US 62 et US 70. Elle pourrait être attribuée à une étape du Néolithique moyen postérieure au Chasséen tel que nous avons proposé de le définir.

\section{ÉLÉMENTS NOUVEAUX DANS LE CHASSÉEN}

La définition du Chasséen que nous avons proposée ci-dessus est fondée sur neuf ensembles clos. Les trois structures 47, 48 et 75 des Bagnoles permettent de renforcer cette définition (tabl. II). Elles apportent également quelques éléments nouveaux.

La présence d'ossements humains fragmentés et brûlés dans les structures FS 47 et FS 48 est un premier point intéressant dans la mesure où elle n'est que rarement signalée dans le Néolithique moyen du midi de la France (Vaquer, 1998, p. 173): le Camp del Ginèbre à Caramany dans les Pyrénées-Orientales; Montbolo/Chasséen, crémation primaire et dépôts d'os brûlés recouvert par une assiette (Vaquer, 2007; Duday, 2007), les Plots à Berriac dans l'Aude (Duday, Vaquer, 2003) et le Vallon de Gaude à Manosque dans les Alpes-de-Haute-Provence (Bérard et al., 1991).

Les éléments les plus inattendus sont sans aucun doute la découverte dans les mêmes structures de deux vases à corps cubique et d'au moins cinq vases peints.

\section{LES VASES À CORPS CUBIQUE}

Les vases à corps cubique sont rares dans le Néolithique d'Europe occidentale. À notre connaissance, dans la région, le seul vase susceptible d'en être rapproché est un petit récipient découvert à l'occasion de fouilles anciennes dans la grotte de Louoï à Vallon-Pont-d'Arc en Ardèche (Guébhard, 1912, pl. 21, no 4). Il s'agit d'un petit vase à panse parallélépipédique aux angles arrondis surmonté d'un col et d'une embouchure ovale (fig. 28, $\mathrm{n}^{\circ} 1$ ). Plus au Nord, un vase à panse cubique provient de la structure XIII U 19 du site de Noyen-sur-Seine en Seine-et-Marne où il est associé à une bouteille attribuée au Chasséen (Henocq-Pochinot, Mordant, 1991, fig. 5). Le seul angle conservé est souligné par un cordon vertical perforé horizontalement dans sa partie supérieure (fig. 28, nº 2). Deux vases à corps cubique proviennent de l'extrême ouest de l'Europe. Un premier a été découvert dans la tombe à couloir du Moulin des Oies à Belz dans le Morbihan (Le Boulaire, Cassen, 2000; Cassen, 2003). Les éléments disponibles permettent de reconstituer un corps cubique d'environ $5 \mathrm{~cm} \times 5 \mathrm{~cm}$ de côté pour une hauteur conservée de $3,5 \mathrm{~cm}$. Les angles sont soulignés par quatre cordons verticaux. L'embouchure n'est pas conservée; cependant, la présence d’un col est suggérée par l'inflexion de la paroi dans la partie supérieure du vase. Il porte un décor composé de six lignes brisées horizontales sur au moins deux des quatre faces verticales du corps dans lequel on pourrait voir une forme simplifiée des décors de triangles quadrillés et de bandes réservées visibles sur les vases des Bagnoles (fig. 28, no 3). Le deuxième vase à corps cubique a été mis au jour dans le paléosol de la tombe à cou- 


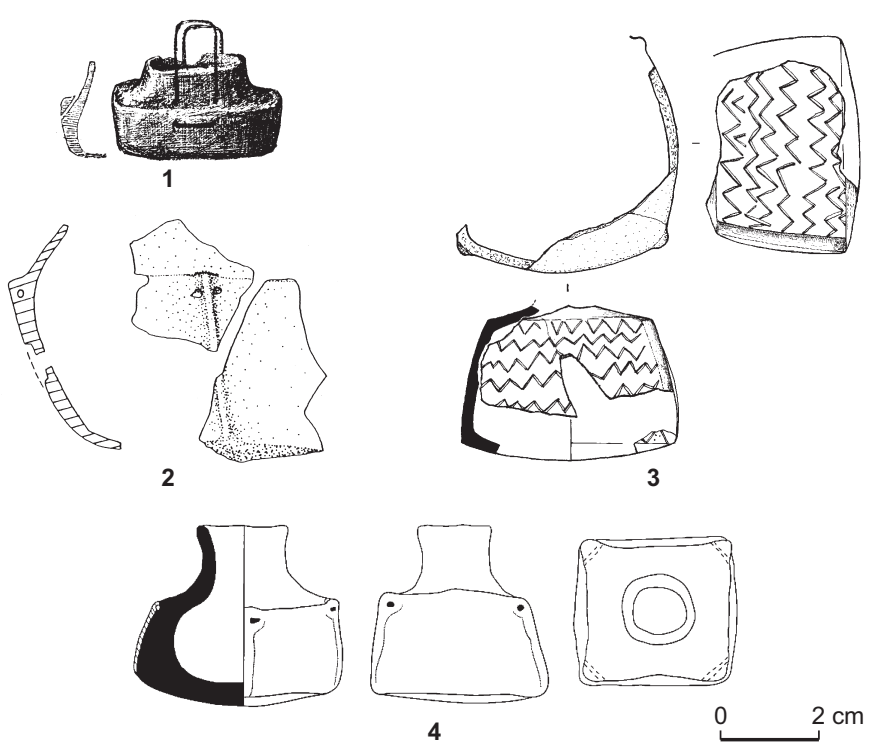

Fig. 28 - Vases à corps cubique d'Europe occidentale: 1, grotte de Louoï à Vallon-Pont-d'Arc en Ardèche (d'après Guébhard, 1912, pl. 21, no 4); 2, structure XIII U19 à Noyen-sur-Seine en Seine-et-Marne (d'après Henocq-Pochinot, Mordant, 1991, fig. 5); 3, moulin des Oies à Belz dans le Morbihan (d'après Le Boulaire, Cassen, 2000); 4, Le Déhus à Guernesey en Grande-Bretagne (d'après Cassen, 2003).

loir du Déhus à Guernesey dans les îles Anglo-Normandes (Mond, Collum, 1933; Cassen, 2003). Il est complet et présente un col légèrement évasé (fig. 28, no 4). Ici encore, les arêtes verticales sont soulignées par des cordons modelés. Ceux-ci sont, à l'instar de l'exemplaire de Noyen, perforés horizontalement dans la partie supérieure. Il ne présentait aucun décor, si ce n'est des empreintes de textile sur le fond. En l'absence de contexte fiable, il est difficile de proposer une datation pour ces deux vases. S. Cassen, s'appuyant sur le fait que l'exemplaire du Déhus a été découvert dans le paléosol d'une tombe à couloir d'un type postérieur à 4300 av. J.-C., propose de le dater entre 4500 et 4200 av. J.-C. (Cassen, 2003, p. 267).

Les assiettes à socle cubique attestées dans le Chasséen sont un autre point de comparaison possible. Les rapports entre les deux types de vases sont cependant limités au volume du corps qui s'inscrit dans un cube. La fonction est par contre fondamentalement différente: socle dans un cas, récipient dans l'autre. Cependant, il faut insister sur le fait que les vases à corps cubique ne peuvent être identifiés comme tels que si leur profil est complet. Il est donc possible que de tels vases, fragmentés et incomplets, aient été interprétés comme des assiettes à socle cubique.
La rareté des parallèles dans le Chasséen et, au-delà, dans l'ensemble du Néolithique d'Europe occidentale, nous a conduit à élargir la recherche à l'Europe centrale, aux Balkans et à l'Italie.

En Europe centrale, certains assemblages de la culture de Lengyel, datée de la seconde moitié du Ve millénaire, ont livré des petits vases en forme de cubes qui présentent aux quatre coins une perforation verticale traversière (fig. 29, $\left.\mathrm{n}^{\text {os }} 1-4\right)$. Ces vases, interprétés comme lampe (Dombay, 1960) ou comme objet cultuel (Bánffy, 1997), se différencient cependant clairement des deux vases des Bagnoles dans la mesure où l'intérieur des vases cubiques Lengyel apparaît sous la forme d'un évidement cylindrique et où ils sont dépourvus de col.

Les parallèles les plus proches sur le plan morphologique proviennent du sud-est de l'Europe. Il s'agit d'une petite série de vases découverts en Roumanie, dans l'ouest de la Bulgarie et en Macédoine. D’une hauteur comprise entre $6 \mathrm{~cm}$ et $9 \mathrm{~cm}$, ils présentent un corps cubique, un épaulement et un col cylindrique. Celui de Tell Priboi à Pernik en Bulgarie attribué au Néolithique ancien (début du VI ${ }^{\mathrm{e}}$ millénaire) est inorné (fig. 29, no 5) (Collectif, 1982). De par sa forme et ses proportions, il est comparable à ceux des Bagnoles. Un autre vase à corps cubique découvert dans un tell de Stara Zagora en Bulgarie a la particularité de présenter deux perforations groupées sous le bord (fig. 29, no 6) (Collectif, 1982). Il est, quant à lui, attribué à la culture de Vesselinovo (Néolithique moyen, fin du VI ${ }^{\mathrm{e}}$ millénaire). Malgré la ressemblance frappante qui existe entre les vases des Bagnoles et les exemplaires bulgares, le décalage temporel, de l'ordre du millénaire, ne permet pas d'établir de liens directs. Cependant, il faut signaler que les vases à corps cubique perdurent dans le sud des Balkans au cours du V $\mathrm{V}^{\mathrm{e}}$ millénaire comme en témoignent les exemplaires de Diakovo à Kioustendil en Bulgarie (fig. 29, no 7) (Krauss, 2001), Zelenikovo en Macédoine (Garašanin, 1979), Ezero en Bulgarie (fig. 29, $\mathrm{n}^{\circ} 10$ ) (Merkyte, 2007) et Gheraieşti en Roumanie (fig. 29, $\mathrm{n}^{\circ} 11$ ) (Draşovean, Popovici, 2008). Ces trois vases sont attribués au Chalcolithique récent local (seconde moitié du Ve et première moitié du IV millénaire). Signalons encore l'existence en Macédoine de figures anthropomorphes à corps cubique ajouré (Tumba, Madžari) (Sanev, 2006, fig. 22 et 25). Un vase à panse cubique a été découvert récemment dans le sud de l'Italie, dans la grotte Carlo Cosma à Santa Cesarea Terme à Lecce (Gorgoglione, 2006). Décoré sur les quatre faces d'incisions curvilignes et d'impressions, ce petit récipient a un corps cubique, un épaulement, une 


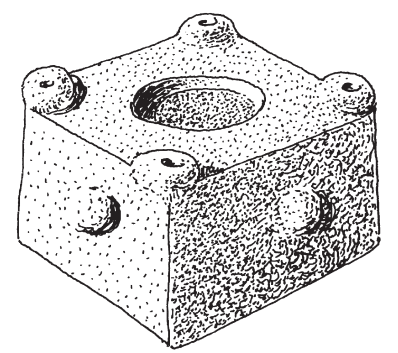

1

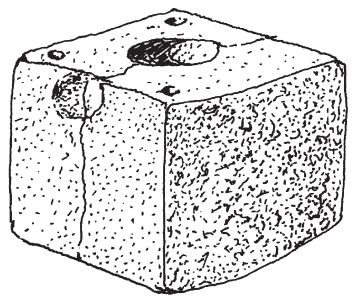

3

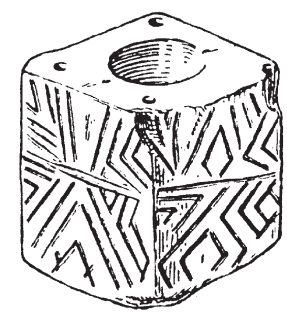

2

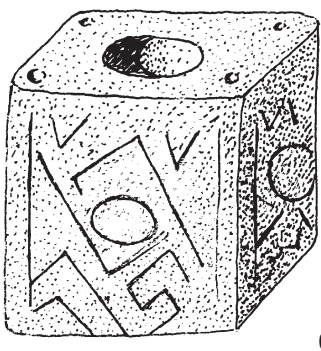

4

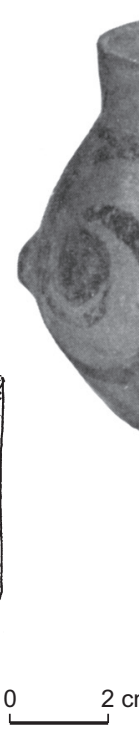

$2 \mathrm{~cm}$

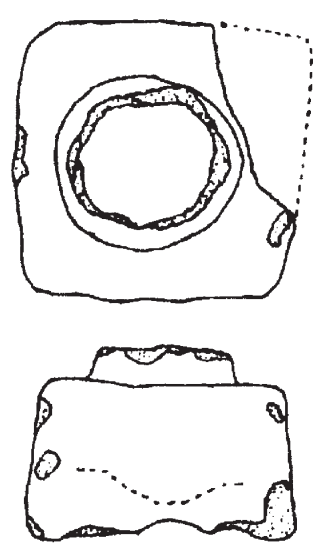

7

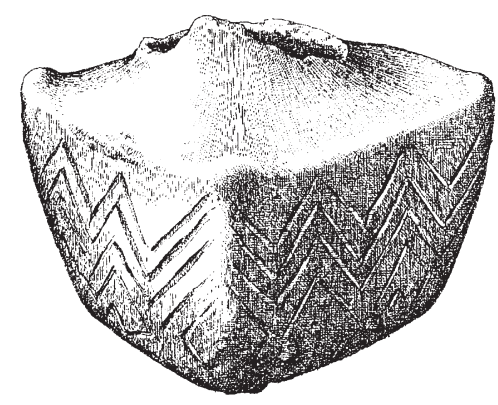

8
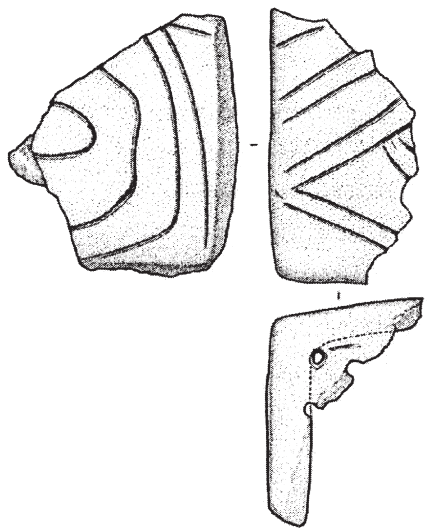

10

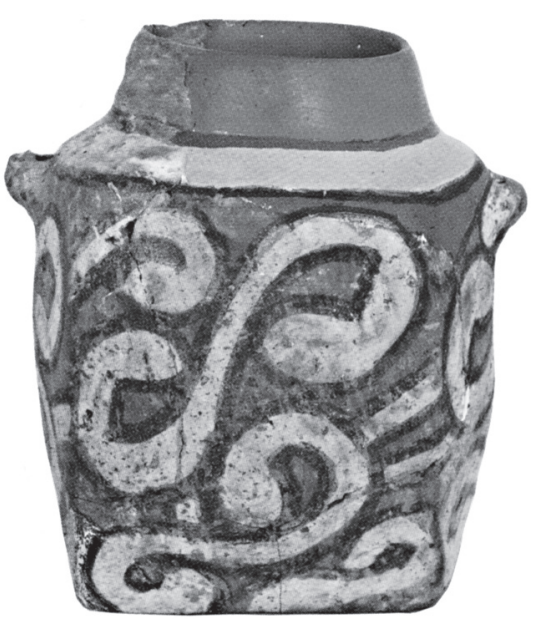

11

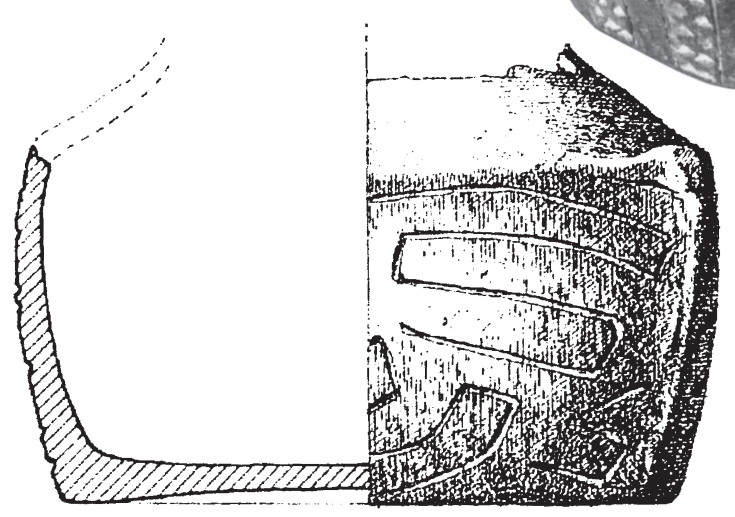

9
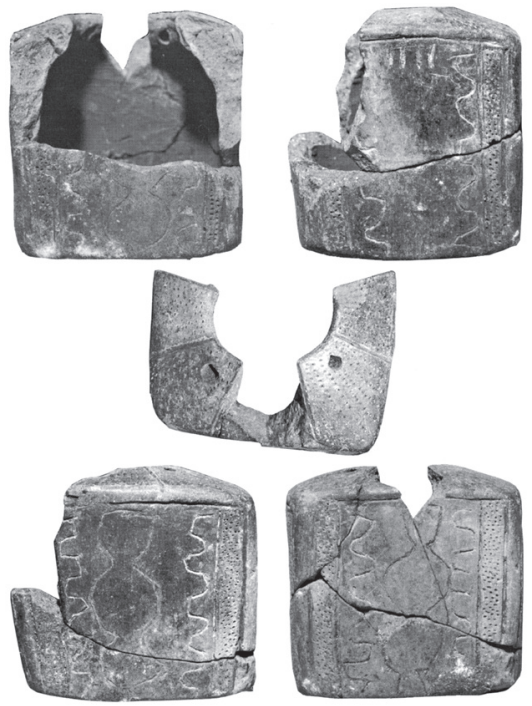

12

Fig. 29 - Différents types de vases à corps cubique en Europe centrale ( $\left.n^{o s} 1-4\right)$, dans les Balkans ( $n^{o s}$ 5-11) et en Italie ( $n^{\circ}$ 10): 1, Santovka en République Tchèque, culture de Lengyel; 2, Lengyel en Hongrie, culture de Lengyel; 3, Aszód en Hongrie, culture de Lengyel; 4, FalkensteinSchanzboden en Autriche, culture de Lengyel (d'après Bánffy, 1997); 5, Tel Priboi à Pernik en Bulgarie, culture de Čavdar-Kremikovci (d'après Collectif, 1982); 6, Stara Zagora en Bulgarie, culture de Vesselinovo (d'après Collectif, 1982); 7, village de Diakovo à Kioustendil en Bulgarie, Chalcolithique (d'après Krauss, 2001); 8-9, Zelenikovo en Macédoine, groupe de Zelenikovo II (d'après Garašanin, 1979); 10, Ezero en Bulgarie, Karavovo VI (d'après Merkyte, 2007); 11, Gheraieşti en Roumanie, Cucuteni B (d'après Draşovean, Popovici, 2008; 12, grotte Carlo Cosma à Lecce en Italie, culture de Serra d'Alto (d'après Gorgoglione, 2006). 
embouchure circulaire sans col et présente au moins deux perforations au-dessus de l'épaulement (fig. 29, no 12). Il est attribué sur des critères typologiques à la culture de Serra d'Alto (milieu du Ve millénaire).

Pour finir, deux vases à panse cubiques proviennent de l'abri sous roche de Su Carroppu dans le sud de la Sardaigne à Sirri dans la province de Carbonia-Iglesias en Italie (Luglié, à paraître). Le premier est décoré de cannelures disposées en zigzags horizontaux, de pieds aux quatre angles du corps du vase et d'au moins une plaquette biforée placée à hauteur de l'épaulement. Le second est inorné, le volume de sa panse s'inscrit dans un parallélépipède rectangle, l'embouchure, ovale, est munie d'une rangée de perforations situées immédiatement sous la lèvre. Dans les deux cas, les parois sont légèrement bombées. Découverts à l'occasion de fouilles clandestines, il est malheureusement impossible de proposer une datation pour ces deux vases. Il est tout au plus possible de mentionner que l'abri sous roche de Su Carroppu a livré un abondant mobilier attribuable au groupe de San Ciriacu qui correspond à la deuxième moitié du $\mathrm{V}^{\mathrm{e}}$ millénaire.

En conclusion, nous proposons de mettre en rapport les vases à corps cubique des Bagnoles avec les exemplaires du même type attestés dans les Balkans et dans le sud de l'Italie $\mathrm{du} \mathrm{VI} \mathrm{C}^{\mathrm{a}} \mathrm{\text {V}} \mathrm{V}^{\mathrm{e}}$ millénaire av. J.-C. Les deux vases cubiques des Bagnoles attestent donc de la présence dans le Chasséen d'un type de vase de tradition balkanique. Leur place dans le Chasséen est encore difficile à évaluer. Ainsi, il est possible d'imaginer que ces vases à corps cubique puissent être représentés dans d'autres assemblages chasséens mais que, étant à l'état fragmentaire, ils aient été systématiquement reconstitués comme assiettes à socle cubique. S'il en était ainsi, les vases à corps cubique du Moulin des Oies et du Déhus, pour lesquels un lien avec les «lampes» de la culture de Lengyel a été proposé (Cassen, 2003), pourraient être alors interprétés comme importations ou imitations de vases à corps cubiques chasséens.

\section{LES VASES PEINTS}

Les vases peints des fosses FS 47 et FS 48 sont difficiles à situer dans le Néolithique d'Europe occidentale. L'utilisation de pigments pour décorer la céramique a été certes occasionnellement signalée dans le Néolithique moyen du midi de la France et du nord de l'Italie: grotte de Camprafaud, couche 14 à Ferrières-Poussarou dans l'Hérault (Rodriguez, 1984, pl. 36, no 4), Puech de la Fontaine à Congénies dans le Gard (Roger, Valette, 1988, fig. 3, $\mathrm{n}^{\circ}$ 3), grotte de Peyroche II à Auriolles en Ardèche (Roudil, Saumade, 1968, fig. 20, $\mathrm{n}^{\circ}$ 11), la Bertaude au Grès d'Orange dans le Vaucluse (Phillips, Courtin, 1981, fig. 11, $\left.\mathrm{n}^{\text {os }} 9-10\right)$, Villa Giribaldi, niveaux $\mathrm{VBQ}$ à Nice dans les Alpes-Maritimes (Binder, 1990, p. 155), grotta del Sanguineto à Finale Ligure en Ligurie (Odetti 2002, pl. XIII, 21 et 22), Arene Candide, niveaux 14, 13 et 8 des fouilles 1972-1977 à Finale Ligure en Ligurie (Tinè, 1999, fig. 26 et 49), niveaux 25 et 23 des fouilles Bernabò Brea (Bernabò Brea, 1956, pl. XII, 1), Arma dell'Aquila à Savona en Ligurie (Bernabò Brea, 1946, pl. XLIX, 8) et la grotte Pollera à Finale Ligure en Ligurie (Bernabò Brea, 1946, pl. XLIX, 9) ${ }^{13}$. Cependant, hormis la présence d'une application de pigment rouge ou brun, ni la forme des supports, ni l'agencement du décor ne sont comparables aux vases des structures FS 47 et FS 48 . Pour ce qui est de la période correspondant au milieu du $\mathrm{V}^{\mathrm{e}}$ millénaire, les cultures à céramique peinte les plus proches de la basse vallée du Rhône sont celles de Ripoli et Serra d'Alto dans le centre et le sud de l'Italie. La céramique peinte de la culture de Serra d'Alto est caractérisée en particulier par des motifs géométriques complexes comme le mobilier des niveaux I-IV de la Cala Colombo à Torre a Mare dans les Pouilles (Lucia et al., 1977) et se distingue clairement des motifs représentés aux Bagnoles. La culture de Ripoli est, quant à elle, caractérisée par une céramique peinte ornée de motifs linéaires: chevrons, triangles hachurés et bandes délimitées par des lignes rouges et remplies de points bruns (voir le site éponyme de Ripoli à Mosciano Sant'Angelo dans les Abruzzes) (Cremonesi, 1965), chevrons et triangles dans la Grotta dei Piccioni à Bolognano dans les Abruzzes (Cremonesi, 1976) et la Grotta Bella à Terni en Ombrie (Guerreschi et al., 1992). Malheureusement, l'état d'avancement des recherches en ce qui concerne la culture de Ripoli ne permet pas actuellement de connaître la place que les décors linéaires y occupent.

Les motifs visibles sur les vases des Bagnoles ne sont pas sans rappeler certains décors de triangles et de chevrons en écorce de bouleau du Cortaillod du plateau Suisse (fig. 30, $\mathrm{n}^{\text {os }} 1-3$ ) parmi lesquels Seematt à Hitzkirch à Lucerne (Wey, 2001, pl. 68, n 5), Egolzwil 2 à Lucerne (Wey, 2001, pl. 22, $\left.n^{\circ} 6\right)$ et Auvernier-Port, couche V à Auvernier à Neuchâtel (Schifferdecker, 1982, pl. 15, no 1). Ce dernier ensemble, attribué au Cortaillod classique, est daté vers 3800-3750 av. J.-C.

13. Les vases peints des Arene Candide, de l'Arma dell'Acqua et de la grotte Pollera sont attribués à la culture de Ripoli. 

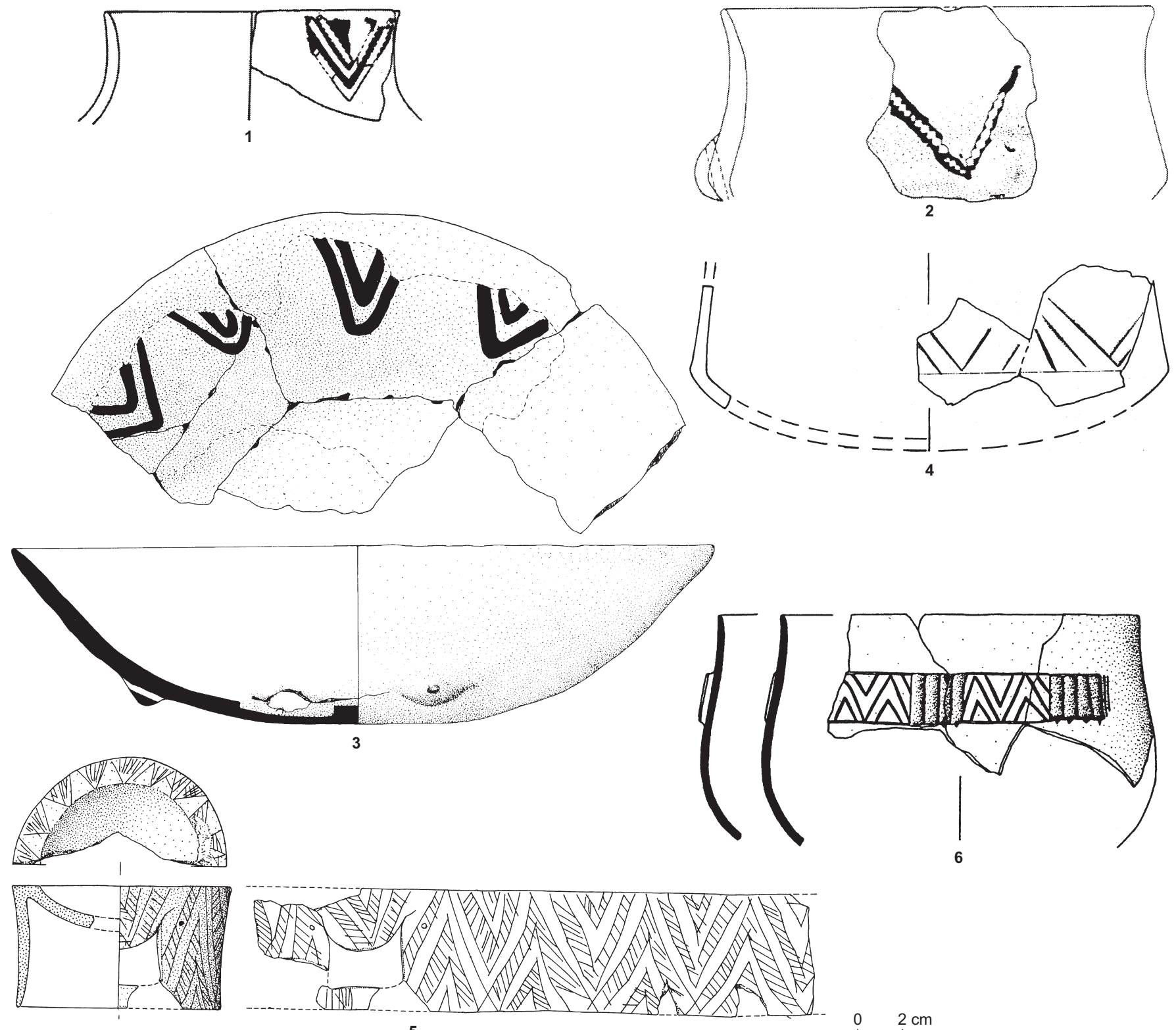

Fig. 30 - Quelques exemples de décors de chevrons de la fin du Vet du début du IV millénaire: 1, Seematt à Hitzkirch en Suisse; 2, Egolzwil 2 à Egolzwil en Suisse (d'après Wey, 2001); 3, Auvernier-Port à Auvernier en Suisse (d'après Schifferdecker, 1982); 4, Arene Candide à Finale Ligure en Italie (d'après Borrello, 1984); 5, Camp de Chassey à Chassey en Saône-et-Loire (d'après Thevenot, 2005, fig. 74, nº 5); 6, Structure 22 de Jacques-Cour II à Montpellier dans l'Hérault (d'après Georjon 2003, fig. 6, nº 2).

Si l'on se limite à la syntaxe du décor sans tenir compte de la technique mise en œuvre, le motif de chevrons incisés est bien attesté en contexte chasséen. Nous ne citerons ici que le décor incisé sur un vase des niveaux chasséens de la grotte des Arene Candide à Finale Ligure en Ligurie (fig. $30, n^{\circ} 4$ ) et les décors incisés de chevrons hachurés sur un vase support du niveau 8 de Chassey (fig. $30, n^{\circ} 5$ ) et sur le bandeau multiforé d'un vase de la structure 22 du site de Jacques-Cœur II (fig. 30, no 6). 


\section{L'ASSIETTE À MARLI ET AILE ÉTROITE ET CONVEXE}

Pour ce type de vase, le meilleur point de comparaison provient du site de Villa Giribaldi à Nice dans les Alpes Maritimes (Binder, 1990, fig. 1, nos 1 et 3). Il s'agit d'une assiette à marli et aile étroite qui est en tout point comparable à l'assiette de la fosse 48 (fig. $31, \mathrm{n}^{\circ} 1$ ). On y retrouve non seulement l'aile étroite et convexe mais aussi un décor sur la paroi externe. Ce vase semble provenir des niveaux chasséens datés vers la fin du V millénaire (Binder, 2004; Gassin et al., 2003). Bien que le décor soit sensiblement différent (décor complexe associant impressions et incisions ou gravure à sec sur le marli, chevrons incisés sur le fond du vase), l'association aile convexe et décor externe sur l'assiette de Villa Giribaldi et sur celle des Bagnoles est frappante. Comme l'a déjà souligné D. Binder (Binder, 1990) à propos de l'exemplaire de Giribaldi, cette forme est bien attestée sur certains sites de la phase méandro-spiralique de la culture des Vases à Bouche Carrée (VBQ II; vers 4400 cal. BC) tels que la Vela à Trente (Bagolini, Biagi, 1976) (fig. 31, $n^{\circ}$ 3) et Casatico à Marcaria en Lombardie (Biagi et al., 1983) (fig. 31, $\mathrm{n}^{\text {os }} 4-5$ ). À ce sujet, une deuxième assiette découverte également à Giribaldi (fig. 31, no 2), mais dont la provenance stratigraphique n'est pas précisée, est intéressante dans la mesure où elle associe une aile convexe à un décor excisé caractéristique de la phase méandro-spiralique de la culture des Vases à Bouche Carrée.

En dehors de Giribaldi et des Bagnoles, le décor recto verso sur assiette à marli est également attesté dans la couche 8B de la grotte de l'Église supérieure à Baudinard dans le Var (Courtin, 1970, fig. 5, no 7) et, en Italie centrale, à la Grotta all'Onda à Camaiore en Toscane (Amadei, Griffoni Cremonesi, 1986-1987, fig. 1, no 9) et la Consuma 1 à Pieve San Stefano en Toscane (Castelletti et al., 1992, fig. 13). Le décor en forme d'étoile à l'extérieur de l'assiette des Bagnoles rappelle certains décors rayonnants du Chasséen méridional tels que ceux figurant sur les assiettes à socle du monument A185 de Saint-Michel-du-Touch (Méroc, Simonnet, 1979, fig. 15L) et de la structure VTC R7-3bis à Villeneuve-Tolosane en Haute-Garonne (François, 2002, pl. 37). Des assiettes à marli et aile étroite et convexe sont connues au sein du mobilier du site du vallon des Vaux à Chavannes-le-Chêne dans le canton de Vaud en Suisse (Sitterding, 1972, pl. 21, nº 5, 9 et 14) et aux Rivaux à EspalySaint-Marcel en Haute-Loire (Daugas, 1986, fig. 4, 9).
L'opération de diagnostic réalisée sur le site des Bagnoles à l'Isle-sur-la-Sorgue a conduit à la découverte d'une série de structures attribuables au Néolithique moyen. La présence dans certains de ces ensembles d'éléments faisant référence au Chasséen nous a amenés à constater l'absence d'une définition claire de cette entité culturelle, ce qui nous a obligés dans un premier temps à revenir aux sources documentaires. Une série d'ensembles clos ou stratifiés unanimement considérés comme chasséens (tabl. II) nous a permis d'établir la liste des caractères céramiques régulièrement associés entre eux et donc caractéristiques de cette culture archéologique. Cette définition repose dans un premier temps sur les traits morphotypologiques de la céramique. Il n'est fait appel aux autres domaines de la culture matérielle (en particulier l'industrie lithique) que dans une deuxième étape du raisonnement.

Défini de la sorte, le Chasséen est caractérisé par des attributs céramiques que l'on retrouve pour la plupart dans les structures FS 13, FS 22, FS 47, FS 48 et FS 75 des Bagnoles: vases à inflexion basse et col rectiligne ou concave, vases à profil galbé, grands vases à bord épaissi à l'extérieur, assiettes à marli et aile rectiligne large, assiettes à socle, coupes en calotte à lèvre droite, coupes en calotte à épaississement interne rectiligne et décrochement vif, jattes à inflexion basse et col rectiligne ouvert et incisions disposées en bandes, triangles ou losanges quadrillés. Sur le plan de l'industrie lithique, le silex blond bédoulien fournit une bonne partie des lames et/ou des outils; les lamelles et petites lames sont principalement débitées par percussion indirecte sur nucléus conique ou semi-conique et les armatures sont majoritairement tranchantes.

Cette définition devrait permettre à moyen terme d'aborder à nouveau, mais sur des bases renouvelées, la question de l'interprétation du Chasséen. À ce sujet, quelques éléments doivent être rappelés ici: il s'agit d'un phénomène court (environ trois à quatre siècles), homogène sur une large région et original dans le contexte du Néolithique d'Europe occidentale.

Les structures FS 47, FS 48 et FS 75 du site des Bagnoles illustrent bien cette originalité et permettent de reposer la question des influences qui ont été à l'origine de sa formation. Ainsi, les vases à corps cubique et les décors peints, éléments qui font probablement partie du corpus chasséen même s'ils n'ont pas été identifiés jusqu'à présent, pourraient évoquer un lien avec les cultures contemporaines de la péninsule Italique et des Balkans. 


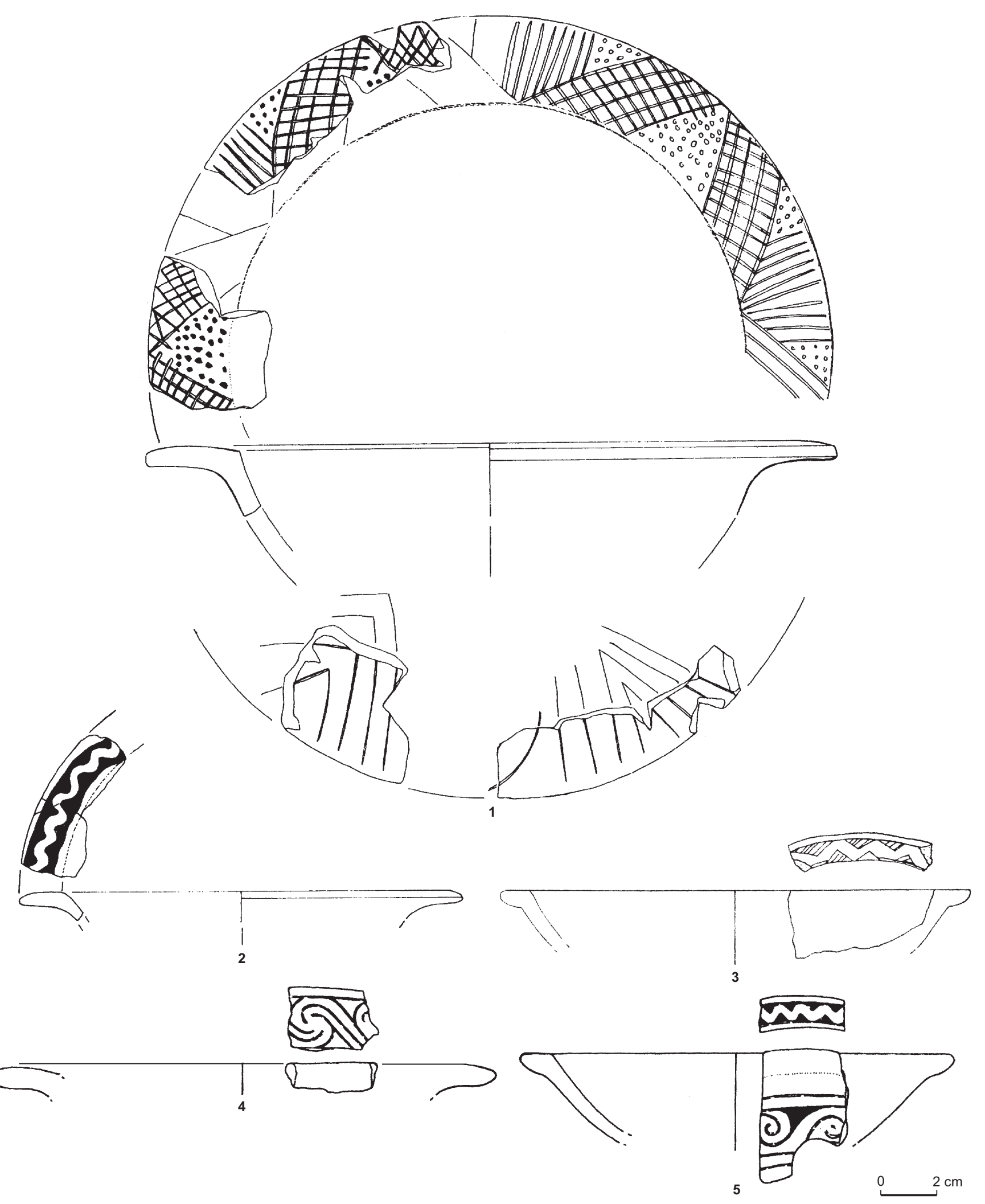

Fig. 31 - Assiettes à marli et aile convexe et types apparentés. 1-2, Villa Giribaldi à Nice dans les Alpes-Maritimes (d'après Binder, 1990); 3, La Vela à Trente en Italie (d'après Bagolini, Biagi, 1976); 4-5, Casatico à Marcaria en Italie (d'après Biagi et al., 1983). 
La définition du Chasséen que nous proposons permet également de poser des questions relatives au sens et à la terminologie des périodes postérieures. En effet, force est de constater que dans le quart sud-est de la France, à l'instar d'autres régions (par exemple la Bourgogne), les principales caractéristiques du Chasséen tel que nous l'avons défini ci-dessus ne sont plus attestées après 4000/3900 av. J.-C. Il serait donc maintenant nécessaire de sortir de l'équation traditionnelle Chasséen $=$ Néolithique moyen = période 4500-3500 av. J.-C. pour se pencher sur la question de l'identité des groupes culturels de la première moitié du IV millénaire.

Nos remerciements vont à E. Arnold, B. Röder et A. Denaire pour leur aide dans le remontage de la céra- mique, à R.-M. Arbogast pour la détermination des restes osseux, à nos collègues A. Reingruber, M.-A. Borello, R. Griffoni-Cremonesi, C. Lichter, L. Thissen, V. Nicolov et I. Matuschik pour avoir orienté nos recherches bibliographiques sur l'Italie, l'Europe centrale et les Balkans, à S. Cassen et à C. Luglié pour avoir attiré notre attention sur les vases cubiques du moulin des Oies, du Déhus et de Su Carroppu, à J.-L. Guendon et à J.-P. Masse pour la détermination pétrographique de la lame de hache de la fosse FS 13 et de la tablette en calcaire de la fosse FS 75 et à C. Cevey qui a réalisé les photographies de la figure 18.

Nous sommes également reconnaissants à J. Vaquer pour sa relecture attentive du texte. Cet article doit beaucoup à ses critiques et conseils avisés. 


\section{BIBLIOGRAPHIE}

\begin{tabular}{ll}
\multicolumn{1}{c}{ ABRÉVIATIONS } \\
ADALR & Association pour le développement de l'archéologie en Languedoc-Roussillon. \\
APRAIF & Association pour la promotion de la recherche en Île-de-France. \\
APDCA & Association pour la promotion et la diffusion des connaissances archéologiques. \\
ARALO & Association pour la recherche archéologique en Languedoc oriental. \\
BAR & British Archaeological Report. \\
BSPF & Bulletin de la Société préhistorique française. \\
SPF & Société préhistorique française. \\
SRA & Service régional d'archéologie.
\end{tabular}

Abélanet J.

1970: «Une tombe néolithique: l'Arca de Calahons (Catllar, Pyrénées-Orientales)», in Les Civilisations néolithiques du midi de la France, Actes du colloque de Narbonne, 1970, Carcassonne, Laboratoire de Préhistoire et de palethnologie, p. 54-55.

Amadei A., Grifoni Cremonesi R.

1986-1987: «La Grotta all'Onda: revisione e inquadramento dei materiali », Rassegna di Archeologia, 6, p. 171-216.

Ambert P., Genna A., TAFfanel O.

1988: «Contribution à l'étude du Chasséen du Minervois », in Le Chasséen en Languedoc oriental, hommage à Jean Arnal, Actes des journées d'études, Montpellier, 25-27 oct. 1985, Montpellier, Université Paul-Valéry, p. 25-36.

Amiel C., JÉdikian G.

2003: «Aspect de la céramique du Chasséen ancien en Languedoc occidental: l'exemple du site d'Encombres à Quarante (Hérault) ", in GAScó J., GUTHERZ X., LABRIFFE P.-A. DE (DIR.), Temps et espaces culturels du $\mathrm{VI}^{e}$ au II $I^{e}$ millénaire en France du Sud, Actes des $I V^{e}$ rencontres méridionales de Préhistoire récente, Nîmes, 28-29 oct. 2000, Lattes, ADALR (coll. Monographies d'archéologie méditerranéenne, 15), p. 389392.

Anzidei A. P., Carboni G.

1995: «Insediamento preistorico di Quadrato di Torre Spaccata (Roma) e osservazioni su alcuni aspetti tardo neolitici ed eneolitici dell'Italia centrale », Origini, 19, p. 55-325.

ARNAL G.-B.

1988: «Le Chasséen dans la région de Lodève", in Le Chasséen en Languedoc oriental, hommage à Jean Arnal, Actes des journées d'études, Montpellier, 25-27 oct. 1985, Université Paul-Valéry, Montpellier, p. $37-42$.

ARNAL J.

1949: «La grotte de la Madeleine (Villeneuve, Hérault) ", $B S P F, 46$, p. 74-75.

1953: «La structure du Néolithique français d'après les récentes stratigraphies", Zephyrus, 4, p. 311-344.

1956: «La grotte de la Madeleine», Zephyrus, 7, p. $33-79$

ARNAL J., BÉNAZET G.

1951: «Contribution à l'étude de la poterie néolithique française", $B S P F, 48$, p. 541564.

\section{BAGOLINI B., BIAGI P.}

1976: «Vela de Trente et le "moment de style adriatique" dans la culture des vases à bouche carrée», Preistoria Alpina, 12, p. 71-77.

BAIlloud G., Mieg DE BoOfZheim P.

1955: Les Civilisations néolithiques de la France dans leur contexte européen, Paris, Picard, $244 \mathrm{p}$.

BAILLS H.

1987: «Éléments pour une chronologie du Néolithique en Catalogne-nord", in GRAU M., POISSON O. (DIR.), Études roussillonnaises offertes à Pierre Ponsich: mélanges d'archéologie, d'histoire et d'histoire de l'art du Roussillon et de la Cerdagne, Perpignan, Le Publicateur, p. 59-68.

\section{BÁNFFY E.}

1997: Cult Objects of the Neolithic Lengyel Culture: Connections and Interpretation, Budapest, Archaeolingua (coll. Serie Minor, 7), $131 \mathrm{p}$.

\section{BEECHING A.}

2002: "La fin du Chasséen et le Néolithique final dans le bassin du Rhône moyen", in FERRARI A., VISENTINI P. (DIR.), Il declino del mondo neolitico: ricerche in Italia centro-settentrionale fra aspetti peninsulari, occidentali e nord-alpini, Atti del convegno, Pordenone, 5-7 avril 2001 Pordenone, Museo delle Scienze (Quaderni del Museo Archeologico del Friuli occidentale, 4), p. 67-83.

BEECHING A., BINDER D., BLANCHET J.-C., Constantin C., DUbouloz J.,

MARTINEZ R., MORDANT D.,

THÉVENOT J.-P., VAQUER J. (DIR.)

1991: Identité du Chasséen, Actes du colloque international de Nemours, 17-19 mai 1989, Nemours, APRAIF (coll. Mémoire du musée de Préhistoire d'Île-de-France, 4), $428 \mathrm{p}$.

BEECHING A., BROCHIER J.-L., CORDIER F., Ferber F., Thiébault S., TREFFort J.-M.

2004: "Aménagement de chenalisations naturelles: un cas original de présence chasséenne à Nyons (Drôme) », in DARTEVElle H. (DIR.), Auvergne et Midi: actualité de la recherche, Actes de la $V$ session des rencontres méridionales de Préhistoire récente, Clermont-Ferrand (Puy-deDôme), 8-9 nov. 2002, Cressensac, éd. du CTHS (Suppl. à Préhistoire du Sud-Ouest, 9) p. 379-394.

BEECHING A., NICOD P.-Y., THIERCELLIN F., VORUZ J.-L.

1997: «Le Saint-Uze, un style céramique non chasséen du $\mathrm{V}^{\mathrm{e}}$ millénaire dans le Bassin rhodanien", in CONSTANTIN C., MORDANT D., SIMONIN D. (DIR.), La Culture de Cerny: nouvelle économie, nouvelle société au Néolithique, Actes du colloque international de Nemours, 9-11 mai 1994, Nemours, APRAIF (coll. Mémoire du 
musée de Préhistoire d'Île-de-France, 6), p. 575-592.

BÉRARD G., BOISSINOT P., GAZENBEEK M. 1991: "Manosque, vallon de Gaude», Bilan scientifique de la région Provence-Alpes-Côte d'Azur, DRAC/SRA, Aix-en-Provence, p. 33-40.

\section{BERNABÒ BREA L.}

1946: Gli scavi nella caverna delle Arene Candide -1- Gli strati con ceramiche, Bordighera, Istituto Internazionale di Studi Liguri (coll. di Monografie Preistoriche ed Archeologiche), $363 \mathrm{p}$.

1956: Gli scavi nella caverna delle Arene Candide (Finale Ligure) -2- Campagne di scavo 1948 1950, Bordighera, Istituto Internazionale di Studi Liguri (coll. di Monografie Preistoriche ed Archeologiche, 292 p.

\section{BEYNEIX A.}

1997: «Les sépultures chasséennes du sud de la France», Zephyrus, 50, p. 125-178.

Biagi P., Barker G., Cresmaschi M.

1983: La Stazione di Casatico di Marcaria (Mantova) nel quadro paleoambientale ed archeologico dell'Olocene Antico della Val Padana Centrale, Istituto Universitario di Bergamo (Studi Archeologici, 2), $135 \mathrm{p}$.

\section{BINDER D.}

1984: «Systèmes de débitage laminaire par pression: exemples chasséens provençaux ", in TIXIER J., INIZAN M.-L., ROCHe H. (DIR.), Préhistoire de la pierre taillée -2- Économie de débitage laminaire, Paris, Cercle de recherches et d'études préhistoriques, p. 71-84.

1990: «Néolithique moyen et supérieur dans l'aire liguro-provençale: le cas de Giribaldi (Nice, Alpes-Maritimes, France) ", in GuIlaine J., GUTHERZ X. (DIR.), Autour de Jean Arnal, Recherches sur les premières communautés paysannes en Méditerranée occidentale, Montpellier, Laboratoire de paléobotanique, p. 147161

1991: «Facteurs de variabilité des outillages lithiques chasséens dans le sud-est de la France", in BEECHING A., BINDER D., Blanchet J.-C., Constantin C., DUBOULOZ J., MARTINEZ R., MoRdant D., THÉVEnOt J.-P., VAQUeR J. (DIR.), Identité du Chasséen, Actes du colloque international de Nemours, 17-19 mai 1989, Nemours, APRAIF (Mémoires du Musée de Préhistoire d'Île-de-France, 4), p. 261-272.

1998: "Silex blond et complexité des assemblages lithiques dans le Néolithique liguro-provençal », in D'ANNA A., BINDER D. (DIR.), Production et identité culturelle, Actes de la $2^{e}$ session des rencontres méridionales de Préhistoire récente, Arles, 8-9 nov. 1996, Antibes, APDCA, p. 111-128.

BINDER D. (DIR.)

2004: Un chantier archéologique à la loupe: Giribaldi, catalogue de l'exposition du 24 oct. 2004 au 25 avril 2005 au Musée archéologique Nice-Cimiez, Nice Musées, $82 \mathrm{p}$.

\section{BINDER D., GASSIN B.}

1988: «Le débitage laminaire chasséen après chauffe: technologie et traces d'utilisation", in BEYRIES S. (DIR.), Industries lithiques: tracéologie et technologie-1-Aspects archéologiques, Archaeopress, Oxford (coll. BAR, International Series, 411-i), p. $93-125$

\section{BINDER D., LEPÈRE C., MAGGI R.}

2008: «Épipaléolithique et Néolithique dans l'arc liguro-provençal: bilan et perspective de recherche", in BINDER D. et al. (DIR.), Archéologies transfrontalières, Alpes du Sud, Côte d'Azur, Piémont et Ligurie: bilan et perspectives de recherche, Actes du colloque de Nice, 13-15 déc. 2007, Monaco, éd. du Musée d'anthropologie et de Préhistoire de Monaco (Bulletin du Musée d'anthropologie et de Préhistoire de Monaco, suppl. 1) p. 49-62.

\section{BORRELLO M.A.}

1984: The Lagozza Culture ( $3^{\text {rd }}$ millennium BC) in Northern and Central Italy, Bergamo, Istituto Universitario di Bergamo (Studi Archeologici, 3), $190 \mathrm{p}$.

BoUTIÉ P. (DIR.)

1988: Le Chasséen en Languedoc oriental, hommage à Jean Arnal, Actes des journées d'études, Montpellier, 25-27 oct. 1985, Montpellier, Université Paul-Valéry, $390 \mathrm{p}$

\section{BRIOIS F.}

2005: Les Industries de pierre taillée néolithiques en Languedoc occidental: nature et évolution des outillages entre les $\mathrm{VI}^{e}$ et $I \mathrm{II}^{e}$ millénaire av. J.-C., Lattes, ARALO (coll. Monographies d'archéologie méditerranéenne, 20), $341 \mathrm{p}$.

\section{BRIOIS F., FURESTIER R., LÉA V.,} RENAULT S.

2008: «Les industries lithiques du midi méditerranéen français et de ses marges aux IV $^{\mathrm{e}}$ et $\mathrm{III}^{\mathrm{e}}$ millénaires», in DIASMEIRINHO M.-H. et al. (DIR.), Les Industries lithiques taillées des $I V^{E}$ et $I I I^{e}$ millénaires en
Europe occidentale, Archaeopress, Oxford (coll. BAR, International Series, 1884), p. 207-230.

\section{BRIOIS F., LÉA V.}

2003: «Productions lithiques autochtones et identité du Chasséen: l'exemple de deux sites de la vallée du Lez (Hérault)", in GASCÓ J., GUTHERZ X., LABRIFFE P.-A. DE (DIR.), Temps et espaces culturels du VI ${ }^{e}$ au $I I^{e}$ millénaire en France du Sud, Actes des IV rencontres méridionales de Préhistoire récente, Nîmes, 28-29 oct. 2000, Lattes, ADALR (coll. Monographies d'archéologie méditerranéenne, 15), p. 135-142.

\section{Cap-Jédikian G., Perrin T.,}

\section{REMICOURT M.}

2008: «Révision des données disponibles sur les aménagements funéraires du site de Saint-Michel-du-Touch (Toulouse, HauteGaronne) », in VAQUER J., GANDELIN M., REMICOURT M. (DIR.), Défunts néolithiques en Toulousain, Toulouse, Archives d'écologie préhistorique, p. 179-196.

\section{Cassen S.}

2003: «Importer, imiter, inspirer ? Objetssignes centre-européens dans le Néolithique armoricain ", L'Anthropologie, 107 , p. $255-270$.

\section{CASSEN S., FRANCOIS P.}

2006: «Du Chasséen armoricain à l'AuzaySandun: un apport de l'ACR 2003-2006 sur le site de la table des Marchands (Locmariaquer, Morbihan) ", Internéo, 6, p. 77-86.

\section{Castelletti L., Martinelli M. C.,} MASPERO A., MORONI A.

1992: «Il sito neolitico della Consuma 1 (Pieve San Stefano, Arezzo) ", Rivista di Scienze Preistoriche, 44, 1-2, p. 43-114.

\section{Claustre F., Ponsich P.}

2000-2001: "Compléments à l'étude de la céramique néolithique de la galerie close de Montou en Roussillon", Études roussillonnaises, 18, p. 79-96.

\section{COLleCtif}

1982: Keramik und Gold:Bulgarische Jungsteinzeit im 6 und 5 Jahrtausend, Catalogue d'exposition édité par le comité culturel de la République populaire de Bulgarie et le musée de Préhistoire de Francfort/Main, $211 \mathrm{p}$.

\section{Courtin J.}

1967: «La grotte de l'Église à Baudinard (Var) », Gallia Préhistoire, 10, p. 282-300. 
1970: «Le Chasséen méridional», in GUILAINE J. (DIR.), Les Civilisations néolithiques du midi de la France, Actes du colloque de Narbonne, 15-17 fév. 1970, Carcassonne, Laboratoire de préhistoire et de palethnologie, p. 27-31.

1974: Le Néolithique de la Provence, Paris, Klincksieck (coll. Mémoires de la SPF, 11), $355 \mathrm{p}$.

\section{Courtin J., PÉlouard S.}

1971: «Un habitat chasséen en HauteProvence: la "grotte C" de Baudinard (Var) », BSPF, 68, p. 540-561.

\section{CREMONESI G.}

1965: «Il villaggio di Ripoli alla luce dei recenti scavi », Rivista di Scienze Preistoriche, 20, p. 85-155.

1976: La Grotta dei Piccioni di Bolognano nel quadro delle culture dal Neolitico all'età del Bronzo in Abruzzo (Collana di Studi Paletnologici, 2), Università di Pisa, $350 \mathrm{p}$.

\section{DAUGAS J.-P}

1986: «Quelques aspects nouveaux du Néolithique du Massif central» in Demoule J.-P., Guilaine J. (DIR.), Le Néolithique de la France, hommage à Gérard Bailloud, Paris, Picard, p. 277-289

\section{DOMBAY J.}

1960: Die Siedlung und das Gräberfeld in Zengővárkony: Beiträge zur Kultur des Aeneolithikums in Ungarn, Bonn, Habelt Verlag, 235 p.

\section{DRAŞOVEAN F., POPOVICI D.N.}

2008: L'Art néolithique en Roumanie, Catalogue d'exposition au Musée historique d'Olten, $230 \mathrm{p}$.

\section{DUDAY H.}

2007: «Discussion autour des coffres», in Moinat P., CHAMbON P. (DIR.), Les Cistes de Chamblandes et la place des coffres dans les pratiques funéraires du Néolithique moyen occidental, Actes du colloque de Lausanne, 12-13 mai 2006, Lausanne/Paris (coll. Cahiers d'archéologie romande, 110 et Mémoires de la SPF, 43), p. 350.

\section{DUDAY H., VAQUER J.}

2003: «Les sépultures chasséennes du site des Plots, Berriac (Aude)", in Chambon P., LECLERC J. (DIR.), Les Pratiques funéraires néolithiques avant 3500 av. J.-C. en France et dans les régions limitrophes, Table ronde de la SPF, Saint-Germain-en-Laye, 15-17 juin 2001, Paris (coll. Mémoires de la SPF, 33), p. 73-79.

\section{FASANI L., VISENTINI P.}

2002: «L'insediamento neolitico e dell'età del Rame di Colombare di Negrar sui Monti Lessini (Verona)", in FERRARI A., VISENTINI P. (DIR.), Il declino del mondo neolitico: ricerche in Italia centro-settentrionale fra aspetti peninsulari, occidentali $e$ nord-alpini, Atti del convegno, Pordenone, 2001, Pordenone, Museo delle Scienze (Quaderni del Museo Archeologico del Friuli occidentale, 4), p. 229-235.

\section{FENOUILLET M., VAQUER J.}

1974: «La fosse néolithique du Lavous, Grand-Gallargues (Gard) », BSPF, 71, p. 231-235.

\section{FRANÇOIS P.}

2002: Les Productions céramiques du Chasséen de Villeneuve-Tolosane: évolution stylistique et comparaisons avec les autres faciès chasséens d'Europe occidentale, Thèse de Doctorat, Toulouse, École des hautes études en sciences sociales, 3 vol.

2007: Les Styles céramiques du Chasséen de Villeneuve-Tolosane, Oxford (coll. BAR, International Series, 1711), $189 \mathrm{p}$.

\section{GADAY R., SARgiano J.-P.}

2006: Les Bagnoles: une occupation du Néolithique moyen à L'Isle-sur-la-Sorgue (Vaucluse), rapport final d'opération, diagnostic, Inrap Méditerranée, Nîmes, 66 p

\section{GARAŠANIN M. (DIR.)}

1979: Praistorija jugoslavenskih zemalja -IINeolitsko Doba, Sarajevo, Akademija nauka i umjetnosti Bosne i Hercegovine, 800 p.

\section{GASSIN B., LUZI C., BEVILACQUA R.}

2003: «Stratigraphie et datation des occupations néolithiques du site de l'usine Chiris (Grasse, Alpes-Maritimes): une contribution à la chronologie du Chasséen provençal», in GASCÓ J., GUTHERZ X., LABRIFFE P.-A. DE (DIR.), Temps et espaces culturels: du $\mathrm{VI}^{e}$ au $\mathrm{II}^{e}$ millénaire en France $d u$ Sud, Actes des IV rencontres méridionales de Préhistoire récente, 28-29 oct. 2000, Lattes, ADALR (coll. Monographie d'archéologie méditerranéenne, 15), p. 401-407.

\section{GEORJON C.}

2003: «Chronologie, variabilité et phénomène de récurrence dans les corpus céramiques chasséens de la vallée du Lez (Hérault) ", in GAScó J., GUTHERZ X., LABRIFFE P.-A. DE (DIR.), Temps et espaces culturels du VI $I^{e}$ au II $I^{e}$ millénaire en France du Sud, Actes des IV rencontres méridionales de Préhistoire récente, Nîmes, 28-29 oct. 2000, Lattes, ADALR (coll. Monographies d'ar- chéologie méditerranéenne, 15), p. 115134.

\section{GORGOGLIONE M.}

2006: «Modello miniaturistico di casa dalla Grotta Carlo Cosma di Santa Cesarea Terme (Lecce)", Rivista di Scienze Preistoriche, 46, p. 186-195.

\section{GUÉBHARD A.}

1912: Sur l'anse funiculaire, Saint-Vallier-deThiey, SPF (coll. Mémoires de la SPF, 2), $184 \mathrm{p}$.

\section{GUERRESCHI G.}

1967: La Lagozza di Besnate e il Neolitico superiore padano, Como, Società Archeologica Comense, $356 \mathrm{p}$.

1976-1977: «La stratigrafia dell'Isolino di Varese dedotta dall'analisi della ceramica (scavi Bertolone 1955-1959)», Sibrium, 13, p. 29-528.

\section{Guerreschi G., CATAlani P., LONGo G.,} IANNONE A.

1992: «Grotta Bella (Terni), una sequenza stratigrafica dal Neolitico inferiore all'età imperiale : i livelli preistorici", Bulletino de Paletnologia Italiana, 83, p. 143-228.

\section{Guilaine J., AMiel C., BarThÈs P.,} COUlarou J., VAQUER J.

1990: "Le Chasséen de l'abri de FontJuvénal», in GuILAINE J., GUTHERZ X. (DIR.), Autour de Jean Arnal, Recherches sur les premières communautés paysannes en Méditerranée occidentale, Montpellier, Laboratoire de paléobotanique, p. 163176.

\section{HENOCQ-POCHINOT C., MORDANT D.}

1991: «La marge sud-est du Bassin parisien: Chasséen et Néolithique moyen SeineYonne", in BEECHING A., BINDER D., Blanchet J.-C., Constantin C., DUBOULOZ J., MARTINEZ R., MORDANT D., THÉVENOT J.-P., VAQUER J. (DIR.), Identité du Chasséen, Actes du colloque international de Nemours, 17-19 mai 1989, Nemours, APRAIF (coll. Mémoires du Musée de Préhistoire d'Île-de-France, 4), p. 199-210.

\section{HOUDRÉ J.-J., VITAL J.}

1979: «Le gisement chasséen ancien du Pirou (commune de Polignac, Haute-Loire)", $B S P F, 76$, p. 355-370.

\section{IRRIBARIA R.}

2006: «La céramique du Chasséen ancien d'Amboise (Indre-et-Loire) ", in DUHAMEL P. (DIR.), Impacts interculturels 
au Néolithique moyen: du terroir au territoire, sociétés et espaces, Actes du XXV colloque interrégional sur le Néolithique, Dijon, 20-21 oct. 2001, Dijon, Société archéologique de l'Est (coll. Suppl. à la Revue archéologique de l'Est, 25), p. 29-43.

\section{JALlOT L., GEORJON C., WATTEZ J.,} BLAIZOT F., LÉA V., BEUGNIER V.

2000: «Principaux résultats de l'étude du site Chasséen ancien de Jacques Cour II (Port Marianne, Montpellier, Hérault)», in LEDUC M., VALDEYRON N., VAQUeR J. (DIR.), Sociétés et espaces, Actes des rencontres méridionales de préhistoire récente, $3^{e}$ session, Toulouse, 6-7 nov. 1998, Toulouse, Archives d'écologie préhistorique, p. 281-304.

KRAUSS R.

2001: «Die prähistorische Siedlung beim Dorf Djakovo, Kr. Kjustendil (Bulgarien) : ein Beitrag zum Äneolithikum im Strumatal», Praehistorische Zeitschrift, 76, p. $129-178$.

LÉA V.

2004a: «Les industries lithiques du chasséen en Languedoc oriental: caractérisation par l'analyse technologique», Oxford, Archaeopress (coll. BAR, International Series, 1232), $215 \mathrm{p}$.

2004b: "Centres de production et diffusion des silex bédouliens au Chasséen », Gallia Préhistoire, 46, p. 231-250

LÉA V., BINDER D., BRIOIS F., VAQUER J. 2007: "Le "Chasséen méridional à lamelles" d'Arnal: évolution de notre perception des industries lithiques ", in ÉVIN J. (DIR.), Un siècle de construction du discours scientifique en Préhistoire: des idées d'hier aux conceptions d'aujourd'hui, Actes du XXVI congrès préhistorique de France, congrès $d u$ centenaire de la SPF, Avignon, 21-25 sept. 2004, Paris, éd. de la SPF, vol. 3, p. 263 275

LÉA V., GEORJON C., LEPÈRE C., SÉNÉPART I., THIRAULT E.

2004: "Chasséen vauclusien qui es-tu ?", in BUISSON-CATIL J. et al. (DIR.), Vaucluse préhistorique, le territoire, les hommes, les cultures et les sites, Le Pontet, éd. A. Barthélemy, p. $163-200$.

\section{Le boulaire A., Gassen S.}

2000: «Le mobilier néolithique de la collection Chaplain-Duparc (1819-1888) : Belz, Erdeven et Plouharnel», in CASSEN S. (DIR.), Éléments d'architecture: exploration d'un tertre funéraire à Lannec er Gadouer (Erdeven, Morbihan). Constructions et recons- tructions dans le Néolithique morbihannais: propositions pour une lecture symbolique, Chauvigny, Association des publications chauvinoises (coll. Mémoire, 19), p. 461482

LUGiA A. DE, Ferri D., Geniola A., Giove C., Maggiore M., Melone N., Pesce-Delfino V., Pieri P., SCATARRELla V.

1977: La Comunità neolitica di Cala Colombo presso Torre a Mare (Bari), Bari, Società di Storia Patria per la Puglia (Documenti e Monografie, vol. XLII), 260 p.

\section{LUGLIÉ C.}

À paraître: «Spinte centrifughe ed interconnessioni di aree periferiche: il Neolitico medio della Sardegna nel quadro delle sue relazioni con la regione padana", in MotTes E. (DIR.), Vasi a Bocca Quadrata - Evoluzione delle Conoscenze, nuovi approcci interpretativi, Convegno di studi, Riva del Garda, dal 13 al 15 maggio 2009.

\section{MERKYTE I.}

2007: «Ezero - Kale from the Copper Age to the Bronze Age in the southern Balkans », Acta Archaeologica, 78, 2, p. 1-78.

MÉroc L., SimonNet G.

1979: «Les sépultures chasséennes de SaintMichel-du-Touch à Toulouse (HauteGaronne) », BSPF, 76, p. 379-407.

MOND R., Collum V.C.

1933: «The Re-Excavation of the Déhus Chambered Mound at Paradis", Vale, Guernesey, Société guernesiaise, p. 1-189.

\section{ODETTI G.}

2002: "Analisi dei materiali ceramici della grotta del Sanguineto o della Matta», Quaderni del Museo Archeologico del Finale, 4, p. 11-69.

\section{Peretto C., TAfFarelli C.}

1973: «Un insediamento del Neolitico recente al Palù di Livenza (Pordenone) ", Rivista di Scienze Preistoriche, 28, 1, p. 236-260.

\section{Phillips P., Courtin J.}

1981: «La Bertaude, le Grès d'Orange (Vaucluse) », BSPF, 78, p. 379-399.

\section{Ponsich P., Treinen-Claustre F.}

1990: «Le gisement néolithique de la galerie close de la grotte de Montou en Roussillon », in GUILAINE J., GUTHERZ X. (DIR.), Autour de Jean Arnal, Recherches sur les premières communautés paysannes en Méditerranée occidentale, Montpellier,
Laboratoire de paléobotanique, p. 101122.

\section{REIMER P.J. ET AL.}

2004: «IntCal04 Terrestrial Radiocarbon Age Calibration", Radiocarbon, 46, 3, p. 10291058 .

RIQUET R.

1959: «Chassey où es-tu ?» BSPF, 56, p. 364374

\section{RODRIGUEZ G.}

1984: La Grotte de Camprafaud: contribution à l'étude du Néolithique en Languedoc central, Montpellier, Office régional de la culture du Languedoc-Roussillon, 433 p.

\section{Roger J.-M., VAletTe $P$.}

1988: «La céramique chasséenne du Puech de la Fontaine (Congénies, Gard): les prospections de la zone centrale, perspectives et premières données ", in $L e$ Chasséen en Languedoc oriental, hommage à Jean Arnal, Actes des journées d'études, Montpellier, 25-27 oct. 1985, Université Paul-Valéry, Montpellier, p. 173-192.

Roudil J.-L., SAumade H.

1968: «La grotte de Peyroche II à Auriolles (Ardèche) ", Gallia Préhistoire, 11, 1, p. 147202.

\section{SANEV V.}

2006: «Anthropomorphic Cult Plastic of Anzabegovo-Vršnik Cultural Group of the Republic of Macedonia», in TASIĆ N., GROZDANOV C. (DIR.), Homage to Milutin Garašanin, Belgrade, Serbian Academy of Sciences and Arts, Macedonian Academy of Sciences and Arts, p. 171-191.

\section{SCHIFFERDECKER F.}

1982: La Céramique du Néolithique moyen d'Auvernier dans son cadre régional, Lausanne, Cahiers d'archéologie romande, 24, 4, $121 \mathrm{p}$.

\section{Servelle C.}

1980: «Deux vases chasséens exceptionnels des Monges (Launaguet, HauteGaronne) ", BSPF, 77, p. 385-396

\section{SITTERDING M.}

1972: Le Vallon des Vaux: rapports culturels et chronologiques, Bâle, Monographie de la Société suisse de Préhistoire et d'archéologie, 20, 160 p.

Tarrús J., Chinchilla J., PONS E.

1982: «La tomba neolítica de "La Bassa" (Fonteta, La Bisbal). Una nova evidéncia 
d'elements Chassey a Catalunya", Barcelona, Informació Arqueológica, 39, p. 59-66.

\section{THEVENOT J.-P.}

2005: Le Camp de Chassey (Chassey-le-Camp, Saône-et-Loire): les niveaux néolithiques du rempart de «la Redoute», Dijon, Société archéologique de l'Est (coll. Suppl. à la Revue archéologique de l'Est, 22), 463 p.

\section{TINÈ S. (DIR.)}

1999: Il Neolitico nella caverna delle Arene Candide (scavi 1972-1977), Bordighera, Genova, Istituto Internazionale di Studi Liguri (coll. di Monografie Preistoriche ed Archeologiche, 10), 620 p.
VAQUER J.

1975: La Céramique chasséenne du Languedoc, Carcassonne, Laboratoire de Préhistoire et de palethnologie (coll. Atacina, 8), $368 \mathrm{p}$.

1990a: Le Néolithique en Languedoc occidental, Paris, CNRS Éditions, 398 p.

1990b: «L'évolution du Chasséen méridional: essai dans le bassin de l'Aude», in GUILAINE J., GUTHERZ X. (DIR.), Autour de Jean Arnal, Recherches sur les premières communautés paysannes en Méditerranée occidentale, Montpellier, Laboratoire de paléobotanique, p. 177-190.

1998: «Les sépultures du Néolithique moven en France méditerranéenne», in Guilaine J. (DIR.), Sépultures d'Occident et genèse des mégalithismes (9000-3500 avant notre ère), Paris, éd. Errance, p. 167-186.

2007: «Les tombes à dalles du Néolithique moyen dans la zone nord pyrénéenne", in Moinat P., Chambon P. (DIR.), Les Cistes de Chamblandes et la place des coffres dans les pratiques funéraires du Néolithique moyen occidental, Actes du colloque de Lausanne, mai 2006, Lausanne/Paris (coll. Cahiers d'archéologie romande, 110 et Mémoires de la SPF, 43), p. 13-25.

\section{WEY O.}

2001: Die Cortaillod-Kultur in der Zentralschweiz: Studien anhand der Keramik und des Hirschoeweihmaterials, Lucerne, Kantonaler Lehrmittelverlag, 204 p. 